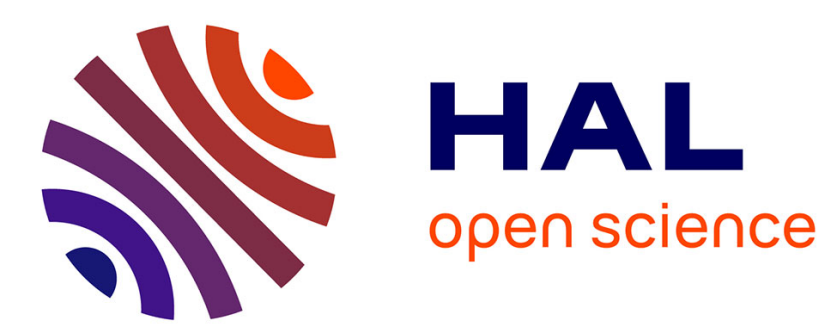

\title{
Understanding human individuation of unfamiliar faces with oddball fast periodic visual stimulation and electroencephalography
}

Bruno Rossion, Talia Retter, Joan Liu-shuang

\section{- To cite this version:}

Bruno Rossion, Talia Retter, Joan Liu-shuang. Understanding human individuation of unfamiliar faces with oddball fast periodic visual stimulation and electroencephalography. European Journal of Neuroscience, 2020, 52 (10), pp.4283-4344. 10.1111/ejn.14865 . hal-02931197

\section{HAL Id: hal-02931197 \\ https://hal.science/hal-02931197}

Submitted on 24 Nov 2020

HAL is a multi-disciplinary open access archive for the deposit and dissemination of scientific research documents, whether they are published or not. The documents may come from teaching and research institutions in France or abroad, or from public or private research centers.
L'archive ouverte pluridisciplinaire HAL, est destinée au dépôt et à la diffusion de documents scientifiques de niveau recherche, publiés ou non, émanant des établissements d'enseignement et de recherche français ou étrangers, des laboratoires publics ou privés. 


\title{
Understanding human individuation of unfamiliar faces with
}

\section{oddball fast periodic visual stimulation and electroencephalography}

Running Title: Understanding face individuation with oddball FPVS

\author{
Bruno Rossion ${ }^{1,2}$, Talia L. Retter ${ }^{3}$, Joan Liu-Shuang ${ }^{4}$
}

1. Université de Lorraine, CNRS, CRAN, F-54000 Nancy, France

2. CHRU-Nancy, Service de Neurologie, F-54000, France

3. Department of Behavioural and Cognitive Sciences, Faculty of Language and Literature, Humanities, Arts and Education, University of Luxembourg

4. Institute of Research in Psychological Science, Institute of Neuroscience, Université de Louvain, Belgium

\section{Corresponding author:}

Bruno Rossion

CRAN UMR 7039, CNRS - Universite de Lorraine,

2 Avenue de la forêt de Haye

54516 Vandoeuvre-lès-Nancy, France.

Tel: $+33(0) 383858053$

E-mail: bruno.rossion@univ-lorraine.fr

131 pages, 21 Figures, 1 Table.

Keywords: face individuation, visual face categorization, EEG, unfamiliar faces, adaptation, frequency-tagging, SSVEP, FPVS 


\section{Abstract}

To investigate face individuation (FI), a critical brain function in the human species, an oddball fast periodic visual stimulation (FPVS) approach was recently introduced (Liu-Shuang et al., 2014). In this paradigm, an image of an unfamiliar "base" facial identity is repeated at a rapid rate $F$ (e.g., $6 \mathrm{~Hz}$ ) and different unfamiliar "oddball" facial identities are inserted every $n^{\text {th }}$ item, at a $F / n$ rate (e.g., every $5^{\text {th }}$ item, $1.2 \mathrm{~Hz}$ ). This stimulation elicits FI responses at $F / n$ and its harmonics ( $2 F / n, 3 F / n$, etc.), reflecting neural discrimination between oddball $v s$. base facial identities, which is quantified in the frequency-domain of the electroencephalogram (EEG). This paradigm, used in 20 published studies, demonstrates substantial advantages for measuring FI in terms of validity, objectivity, reliability, and sensitivity. Human intracerebral recordings suggest that this FI response originates from neural populations in the lateral inferior occipital and fusiform gyri, with a right hemispheric dominance consistent with the localization of brain lesions specifically affecting facial identity recognition (prosopagnosia). Here we summarize the contributions of the oddball FPVS framework towards understanding FI, including its (a)typical development, with early studies supporting the application of this technique to clinical testing (e.g., Autism Spectrum Disorder). This review also includes an in-depth analysis of the paradigm's methodology, with guidelines for designing future studies. A large-scale group analysis compiling data across 130 observers provides insights into the oddball FPVS FI response properties. Overall, we recommend the oddball FPVS paradigm as an alternative approach to behavioral or traditional event-related-potential EEG measures of face individuation. 


\section{Index}

ABSTRACT

INDEX. 3

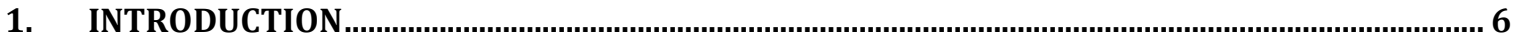

2. THE IMPORTANCE OF HUMAN UNFAMILIAR FACE INDIVIDUATION...................................... 8

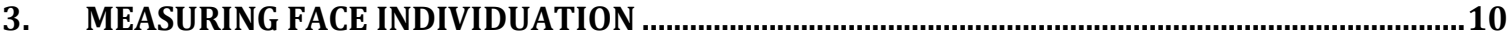

3.1. THE DESIRED VIRTUES OF A FACE INDIVIDUATION MEASURE........................................................................ 10

3.2. THE DIFFICULTIES OF EXPLICIT BEHAVIORAL MEASURES …...........................................................................11

3.3. THE DIFFICULTIES OF EVENT-RELATED-POTENTIAL MEASURES OF FACE INDIVIDUATION ................................... 16

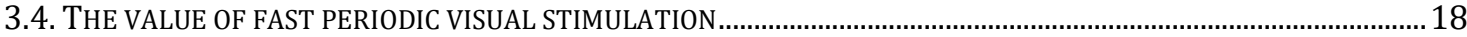

4. FACE INDIVIDUATION WITH AN ODDBALL FPVS PARADIGM AND EEG ...............................19

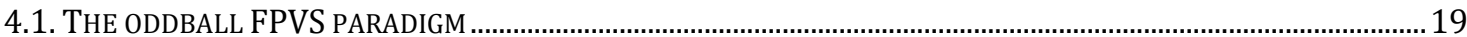

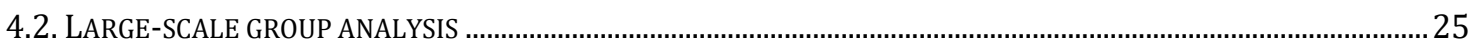

5. ADVANTAGES OF THE APPROACH

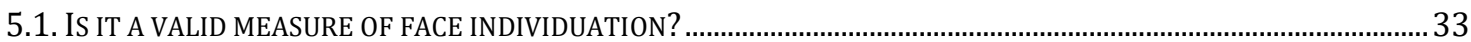

5.1.1. Discrimination and generalization, automaticity, and time-constraints.................................. 33

5.1.2. A high-level FI response despite identical base face images? ......................................................... 34

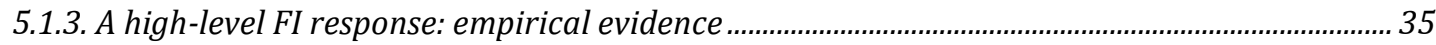

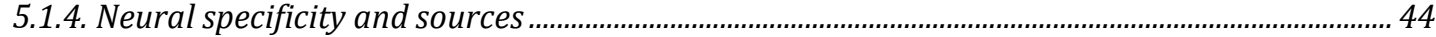

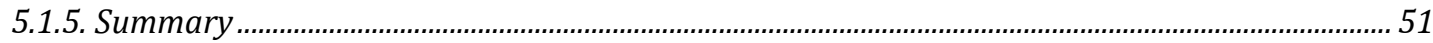

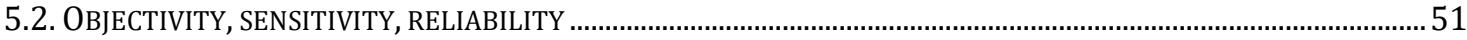

5.2.1 Objectivity in FI response identification and quantification .......................................................... 51

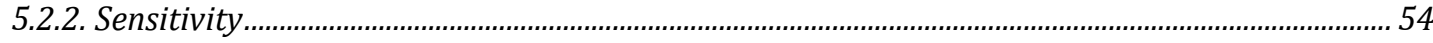

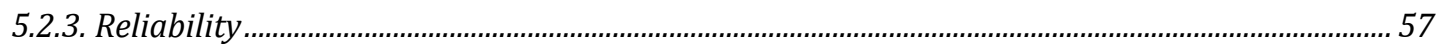

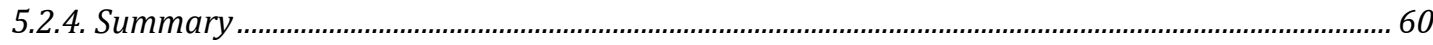

6. MECHANISMS

6.1. GENERAL NEURAL MECHANISMS OF FREQUENCY-TAGGED EEG RESPONSES.................................................... 60 


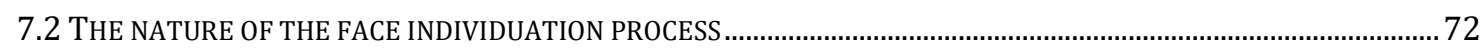

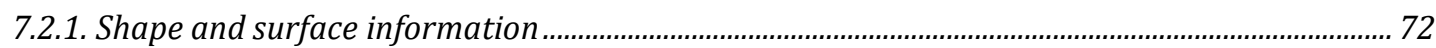

7.2.2. Holistic individuation of faces through the composite face effect.............................................. 72

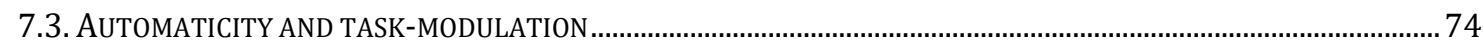

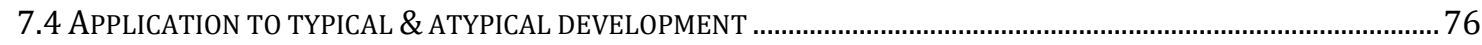

7.4.1. Typical development

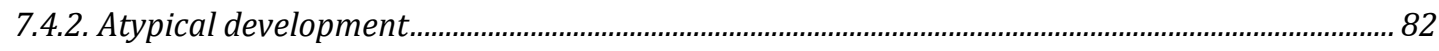

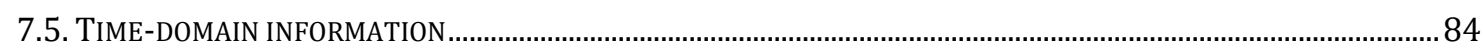

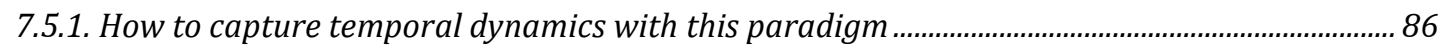

7.5.2. Insights into the temporal dynamics of face individuation ............................................................ 86

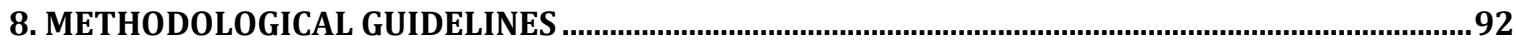

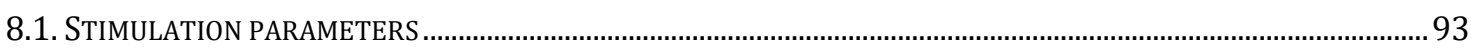

8.1.1. Selecting and controlling the stimulus set.................................................................................... 93

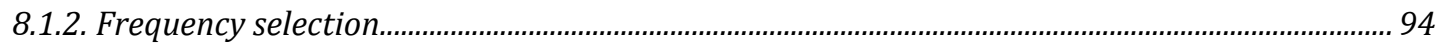

8.1.3. Stimulation sequence and overall recording duration ................................................................ 97

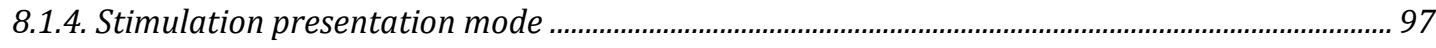

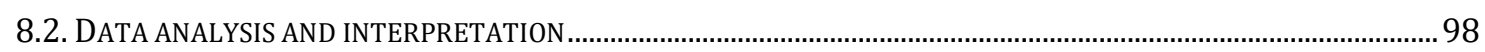

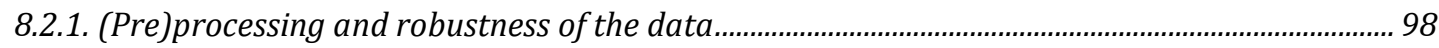

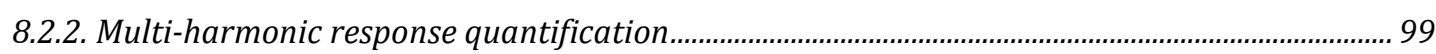

8.2.3 Interpreting FI response amplitude differences ........................................................................ 100

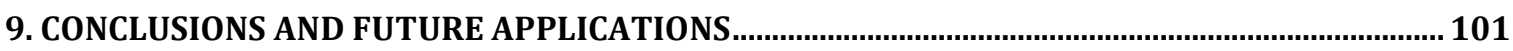

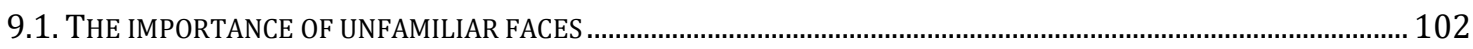

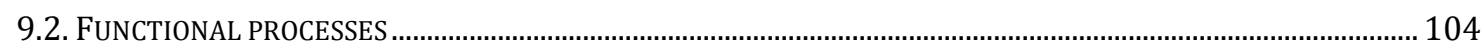

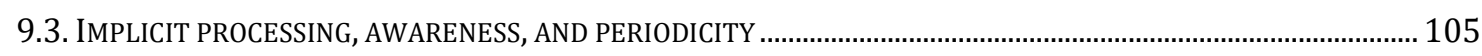

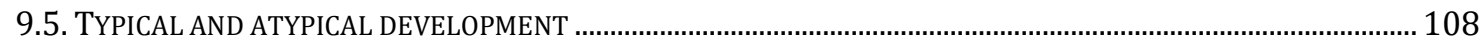


Rossion, B., Retter, T.L., Liu-Shuang, J. (2020). European Journal of Neuroscience, in press.

DATA SAMPLE. 110

FREQUENCY-DOMAIN ANALYSIS 110

Quantification of the (oddball) face individuation response. 111

Quantification of the general visual response.... 111

TIME-DOMAIN ANALYSIS 112

ACKNOWLEDGMENTS. 


\section{Introduction}

Here we review the research performed over the past few years with a paradigm in which images of one unfamiliar human facial identity are repeated at a rapid fixed rate and interspersed at regular intervals with images of different unfamiliar facial identities (Figure 1). Neural (typically electrophysiological) recordings during this fast periodic visual stimulation (FPVS) mode reveal a response at the frequency of identity change that reflects a critical brain function in the human species: face individuation (FI). Our goals in this review are to (1) illustrate how we can use this approach to probe FI in the human brain, essentially as an alternative or complementary measure to behavioral and standard event-related potential (ERP) methods, and to (2) provide a better understanding of human FI through this paradigm.

\section{A Face presentation frequency $=6 \mathrm{~Hz}$}

Face identity change frequency $=1.2 \mathrm{~Hz}(1 / 5)$
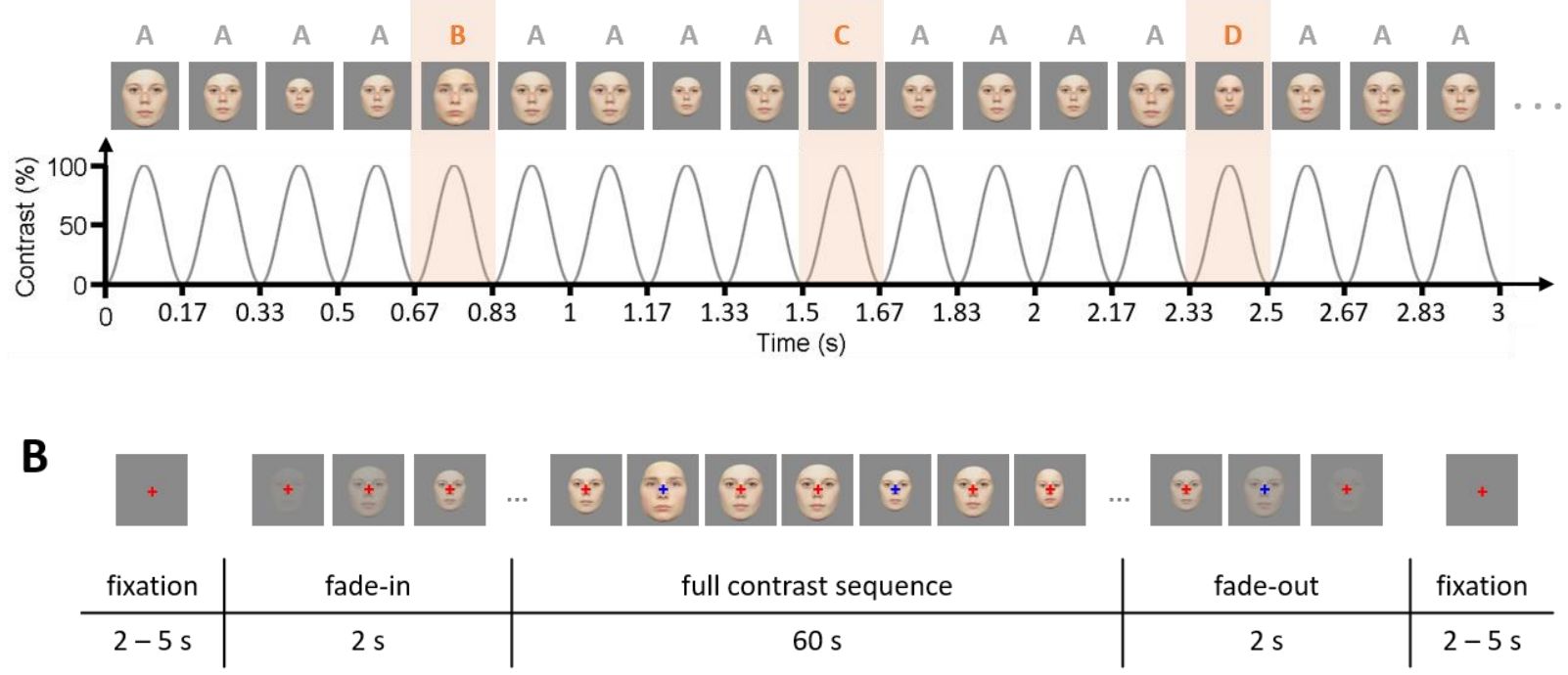

Figure 1. The oddball fast periodic visual stimulation (FPVS) face individuation paradigm.

A. Schematic illustration of the paradigm (first reported in Liu-Shuang et al., 2014) in which one repeating unfamiliar human facial identity is presented through sinusoidal contrast modulation at a $6 \mathrm{~Hz}$ rate, and different unfamiliar facial identities of the same sex are inserted as every fifth stimulus (i.e., 1.2 Hz). (See also a short stimulation movie here: https://face-categorization-lab.webnode.com/products/aaabstimulation/) The repeated facial identity is usually randomly selected from a large pool of unfamiliar faces (Figure 2) and changes every stimulation sequence. While neural activity at $6 \mathrm{~Hz}$ and its harmonic frequencies (12 Hz, etc.) reflects the general response to visual (face) stimulation, a response at $1.2 \mathrm{~Hz}$ and its specific harmonic frequencies $(2.4 \mathrm{~Hz}$, etc.) is taken as a measure of face individuation. B. Typical stimulation sequence structure. A sequence is usually presented for about one minute during EEG recording. 
In most studies, it is flanked by a $2-5 \mathrm{~s}$ fixation period without stimulation, followed by $2 \mathrm{~s}$ in which the maximal contrast of the face stimuli gradually ramps up/down. These time windows aim to reduce artifacts related to the sudden onset/offset of flickering stimuli and are not included for response quantification. Throughout the stimulation sequence, participants typically perform an orthogonal task in which they detect rare (6-8), random color or shape changes of the central fixation cross.

At a theoretical level, we consider understanding human FI and its neural basis as a step towards understanding the more general human brain function of categorization, or recognition, which is key for a biological organism's successful interaction with the environment. To achieve categorization/recognition, the central nervous system must be able to provide different responses to different sensory inputs (i.e., discriminate) and identical or similar responses to the same input appearing under different viewing circumstances (i.e., generalize). Individual human faces provide an ideal type of signal to investigate categorization/recognition in the human brain since: 1) they are complex visual stimuli (i.e., they vary in terms of multiple visual cues interpreted in terms of shape, texture and color, both at the level of their local features and the relative positions of these features); 2) they are visually homogenous and yet objectively different, even when external features are removed (Figure 2); 3) faces are ubiquitous in our natural and digital environments, and play a central role in social interactions; 4) they need to be categorized/recognized at the level

\footnotetext{
${ }^{1}$ In a standard cognitive framework that draws a sharp border between the extraction of (a) visual representation(s) of a stimulus and its representation(s) in memory, "categorization" and "recognition" are often used to refer to different processes/functions (i.e., "perceptual categorization" is generally thought to rely on objective physical properties of the stimuli, while "recognition" would involve the comparison to a mnesic representation derived from past experience). In the present framework, we consider instead the terms "categorization" and "recognition" as synonyms, referring to the central nervous system' production of a response to an environmental stimulus that is both selective (i.e., discriminant; behavioral or neural) and reproducible (i.e., generalizable) across different instances of that stimulus. In this theoretical framework, "perception" is defined as the subjective (conscious) experience of recognition (see Rossion \& Retter, 2020).
} 
of individual exemplars in the human species; and, 5) their level of familiarity can vary immensely within observers, across observers, and across time.

At a methodological level, various forms of so-called "oddball" paradigms such as the one reviewed here have been used extensively in cognitive neuroscience research (since Ritter et al., 1968 in the auditory domain; Ritter \& Vaughan, 1969 for both auditory and visual stimulation). There is also a great deal of debate and interest regarding the type of (electrophysiological) responses elicited in such designs and the neural mechanisms that they reflect. We consider that and will discuss how - these issues can benefit from a review focused on the measure of FI with FPVS.

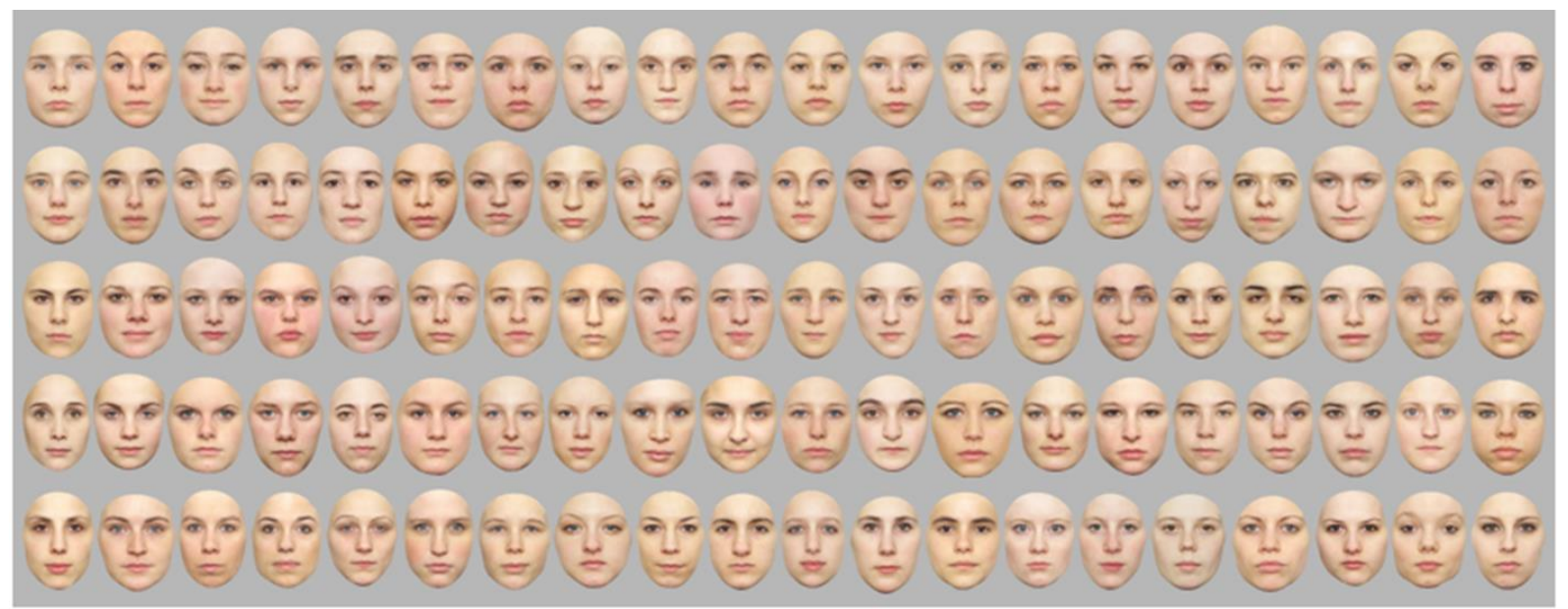

Figure 2. Example of a set of homogenous individual female faces used in face individuation paradigms (from Retter et al., in preparation).

Pictures are taken under the same condition in terms of head pose, expression, and lighting conditions, and cropped for external features while preserving face size (Laguesse et al., 2012). The stimuli are typically presented in color, and sometimes equalized in terms of global luminance. Despite the absence of external features in this relatively homogenous set, the unfamiliar facial identities are clearly distinguishable for neurotypical adults.

\section{The importance of human unfamiliar face individuation}

This review focuses on human face individuation (FI) of unfamiliar facial identities.

Investigating FI in humans is particularly important, because expertise at FI may be unique to the human species. Although many non-human animal species, such as sheep, fish, wasps, or bees, can be trained through operant conditioning to behaviorally individuate pictures of faces of 
conspecifics or even of humans (Peirce et al., 2001; Newport et al., 2016; Sheehan \& Tibbetts, 2011; Dyer et al., 2005), their performance is limited, and heavily dependent on image-specific features, often external to the face (e.g., antennae or ears, see Sheehan \& Tibbetts, 2011; Peirce et al., 2001, respectively). This also applies to macaque monkeys, an animal species that is considered by many neuroscientists as a very good model of the human visual system, and face processing in particular (e.g., Di Carlo \& Cox, 2007; Tsao et al., 2008). In reality, macaque monkeys perform poorly at relatively simple behavioral FI tasks, their performance differs qualitatively from humans (i.e., they depend on image-specific features and are not specifically affected by stimulus inversion and long-term familiarity), and they do not possess a human-like cortical circuitry for efficient FI (Parr, 2008; Rossion \& Taubert, 2019; Griffin, 2020, for reviews).

There are at least two main reasons why FI is more important for humans than other animal species. First, in humans, individuation is based primarily on the face, which is clearly visible during most interactions and shows elevated phenotypic and genetic inter-individual variability compared to other body parts (Sheehan \& Nachman, 2014). Second, humans constantly view numerous unfamiliar (as well as familiar) faces: most human societies are characterized by the presence of a large group of individuals and fission-fusion dynamics, i.e., a tendency to change (the number of) experienced individuals over time. In modern human societies, individuals are further exposed to thousands of additional facial identities through digital media. In these conditions, compared to other species, neurotypical human adults truly excel at individuating faces of conspecifics (Rossion, 2018a). Importantly, this ability is "learned naturally"; young children's face individuation abilities appear limited (at least as measured in behavioral tasks) but they improve spontaneously during development, in the absence of formal training (e.g., Carey, 1992; Mondloch et al., 2006; Hills \& Lewis, 2018; Mardo et al., 2018). Human FI is also fast (e.g., within 1-2 fixations, Hsiao \& Cottrell, 2008; emerging around $160 \mathrm{~ms}$ after stimulus onset, Rossion \& Jacques, 2011), and automatic (i.e., occurring without the intention to do so and without the ability to be suppressed; Palermo \& Rhodes, 2007; Yan et al., 2017). 
Although it is clear that long-term familiarity with specific facial identities substantially improves our behavioral ability to individuate these faces (e.g., Bruce, 1982; Bruce et al., 2001; Jenkins et al., 2011; see Ramon \& Gobbini, 2018; Young \& Burton, 2018 for reviews), our ability to individuate unfamiliar faces is particularly important, since picking up the idiosyncratic visual features of faces (Figure 2) when we first encounter them forms the basis for subsequently recognizing the face of a familiar person. Indeed, even for unfamiliar faces, FI implies that the specific neural response elicited by the face of a unique individual can be repeated reliably although not necessarily identically - when this individual is encountered again. Moreover, contrary to familiar faces, individuation of unfamiliar faces cannot be based on encoded multimodal associations (i.e., semantics), allowing the isolation and thus better characterization of the visual processes involved in this function (Rossion, 2018a).

\section{Measuring face individuation}

\subsection{The desired virtues of a face individuation measure}

Characterizing FI in neurotypical human adults is an important goal of cognitive neuroscience research and requires addressing many questions: which stimulus properties drive face individuation?; how are facial features combined into integrated visual representations?; how fast is FI?; how much does it depend on attentional processes?; etc. Ultimately, we aim to understand human FI in terms of its neural mechanisms, and to characterize how this function develops in the normal population and how it is affected in neurological and neuropsychiatric conditions.

To achieve these objectives, a proper measure of human FI must fulfill a number of criteria. First and foremost, it must be valid, i.e., it must measure a specific brain function that is behaviorally relevant in the real world. Consequently, even though explicitly recognizing familiar facial identities in a natural environment depends on many factors beyond FI, the FI measure should be able to clearly identify someone who has marked difficulties at recognizing facial identities in 
real life. Second, this measure must be reliable, i.e., stable over time at both the group and individual level. Reliability is the pedestal of scientific research, since it is key for replicating results and for building solid knowledge. Reliability is particularly important in clinical and developmental studies, which aim to identify genuine impairments and the specific evolution of a function across time, respectively. Yet, perhaps because of the constant drive for novelty in cognitive neuroscience research, the reliability of behavioral and neural measures is often neglected. The FI measure should also be as objective as possible, leaving little room for subjective methodological decisions leading to variability in the same measure taken by different experimenters. Last but not least, the measure should be highly sensitive, i.e., clearly discriminating the process of interest (FI) from noise in a minimal amount of time. This latter point is especially critical in the context of clinical and developmental studies.

\subsection{The difficulties of explicit behavioral measures}

Traditionally, unfamiliar FI is measured in terms of the accuracy (and response time) of observers asked to judge whether two pictures represent the same facial identity or not, or to match two or more pictures of the same facial identity presented alongside different distractor identities. Hundreds of studies have reported such experimental measures of FI since their appearance in the mid-1960s, initially as paper-and-pencil assessment tools for patients with occipital and temporal brain damage (de Renzi \& Spinnler, 1966; Benton \& Van Allen, 1968; Milner, 1968). Subsequently, researchers developed behavioral tests with schematic faces or photographs of unfamiliar faces in order to evaluate and characterize FI ability in neurotypical populations (e.g., Yin, 1969; Laughery et al., 1971; see Ellis, 1975 for a review of early studies). Many laboratories developed a variety of computerized assessments of this function in the following decades, and a number of these digital tests are now widely available, together with normative data (e.g., the Cambridge Face Memory Test (CFMT): Duchaine \& Nakayama, 2006; Bowles et al., 2009; see also Russell et al., 2009 for a longer version of the test, the CFMT+; the Cambridge Face Perception 
Test (CFPT): Duchaine et al., 2007; the Glasgow Face Matching Test (GFMT): Burton et al., 2010; the Caledonian Face Test: Logan et al., 2016; the Kent Face Matching Test (KFMT): Fysch \& Bindemann, 2018; and the computerized version of the Benton Face Recognition Test (BFRT): Rossion \& Michel, 2018, adapted from the original test of Benton \& Van Allen, 1968). However, whether these behavioral tests possess the virtues listed above for adequately measuring FI, especially in terms of high validity, is questionable.

The validity of these tests is problematic foremost because explicit behavioral performance at any face recognition test depends on many general factors besides FI, namely task understanding, motivation, general visual memory encoding, short-term memory maintenance and retrieval, spatial attention, visual search, decision making, motor coordination, etc. Hence, low accuracy, or particularly long response time, at these behavioral tests may not necessarily imply poor FI ability. This may particularly be the case for performance at the widely used CFMT, a (purposefully) very difficult test involving many of the cognitive processes listed above (see Duchaine \& Nakayama, 2006; Russell et al., 2009 for descriptions of the test requirements). For instance, although it has been claimed that the CFMT captures a highly domain-specific ability (Wilmer et al., 2012), performance at this test depends on general intelligence, and more specifically short-term memory and lexical knowledge ability (Gignac et al., 2016). Performance and speed at other explicit behavioral face recognition tests also depend considerably - although not completely - on general cognitive abilities (Wilhem et al., 2010; Hildebrandt et al., 2011). As for the BFRT, it has long been known that brain-damaged patients with substantial impairment for language comprehension show a notably high frequency of defect at this test (Hamsher et al., 1979). As a consequence of this uncertain validity, it is unclear whether the large inter-individual variability observed with these tests (e.g., in the CFMT: Bowles et al., 2009; Palermo et al., 2017; CFMT \& GFMT: McCaffery et al., 2018; BFRT: Rossion \& Michel, 2018) or with other explicit behavioral measures of FI (e.g., Wilhem et al., 2010; Bindemann et al., 2012; Estudillo \& Bindemann, 2014; Stacchi et al., 2020) in the normal population reflects genuine variability at the FI function or variability of 
these general factors ${ }^{2}$. Since performance is only modestly correlated across different behavioral tests in the normal adult population (e.g., Wilhem et al., 2010; McCaffery et al., 2018; Stacchi et al., 2020), the weight of general factors involved in these various tasks could be predominant.

Even when the task is simplified to simultaneous unfamiliar face matching in order to reduce the contribution of general cognitive factors, as in the GFMT (Burton et al., 2010), these validity issues remain, and can be exacerbated by stimulus selection and control. For instance, stimuli are often selected to artificially regulate similarity, which sometimes differs between targets and distractors to decrease performance and avoid ceiling effects (e.g., a selection of face pictures of the same target individual that are markedly different and face pictures of different distractor individuals that are highly similar in the GFMT, see Bruce et al., 1999) (see also Duchaine et al., 2007; Logan et al., 2016).

Importantly, these explicit behavioral tests are further limited in their ability to validly capture two key aspects of FI: automaticity and rapidity. While FI is sometimes performed explicitly in a natural setting (e.g., when actively searching for somebody in a crowd), typical human adults generally individuate faces automatically, i.e., unintentionally and involuntarily. In fact, spontaneously recognizing the face of an unexpectedly encountered familiar person ("the butcher-in-the-bus" phenomenon) is perhaps the most challenging operation, and failure to do so is a common mistake reported by neurological patients with prosopagnosia. Although we may seldom be conscious of individuating unfamiliar faces in natural settings, we nevertheless recognize unfamiliar faces as unfamiliar, and we readily notice when this process is challenged,

\footnotetext{
${ }^{2}$ Attempts to control for inter-individual variability of other factors can be made by asking participants to run the same behavioral task with another material, e.g., pictures of cars (Dennett et al., 2012) and normalizing the data obtained on faces by the data obtained with pictures of the other material. However, this procedure assumes that the general factors are independent of the material used, which is unlikely. Most importantly, instead of eliminating them, such procedures are likely to introduce additional sources of inter-individual variability in the measures.
} 
e.g., when we see faces of identical twins for the first time. Automaticity is not measured when an explicit behavioral task is imposed. Moreover, such non-automatic tasks may introduce response biases (e.g., being influenced by feedback) that vary across populations (e.g., White et al., 2014).

In regards to rapidity, in a real-world social setting, FI must take place within a few hundreds of milliseconds to facilitate smooth interactions with others. Yet, most behavioral tests, as referred to above, typically use long stimulus presentation durations that do not account for speed of processing, because time pressure in explicit unfamiliar FI tasks can deteriorate behavioral performance even in healthy adult participants (Bindemann et al., 2016; Fysh \& Bindemann, 2017), and would be particularly problematic when testing children or clinical populations (e.g., Powell et al., 2019). Additionally, the absence of time constraints in these tests could also favor unnatural, analytical processing of the face images or other deviant task strategies (e.g., focusing exclusively on the mouth or hairline). Sensitivity is also limited in behavioral tests that do not consider response time, which certainly carry relevant information about FI ability (Wilhelm et al., 2010; Rossion \& Michel, 2018; but see Meyer et al., 2019). However, accuracy and response time are difficult to combine or interpret together (Bruyer \& Brisbaert, 2011; Liesefled \& Janczyk, 2019).

For all these reasons, despite their usefulness in fundamental research and clinical evaluation, behavioral tests relying on explicit instructions and observer responses are limited at adequately capturing the FI function. This makes it particularly challenging to compare FI ability between individuals (see Stacchi et al., 2020), as well as to assess this ability across development and in clinical populations. For instance, these tests are often not suitable to capture the development of FI in children: instead of being used in their original format, they have to be adapted to test children from 5 years of age (e.g., the "CFMT-C", Croydon et al., 2014), yet again, general developmental factors and stimulus limitations could still heavily influence performance (Croydon et al., 2014). These limitations also impair behavioral tests' usefulness to understand the neural basis of the FI function. For instance, an extensive lesion-deficit mapping technique on a large group of focal brain-damaged patients performing the BFRT points primarily to the posterior- 
inferior parietal region (specifically, in the angular gyrus), i.e., a region that is typically not associated with prosopagnosia but may be critical to support (visuospatial) attention and visual search processes that are required for the BFRT (Tranel et al., 2009) ${ }^{3}$.

The limitations of behavioral tests have led to endless scientific debates and contradictions in the field of human face recognition. Most significantly, while the vast majority of studies use pictures of unfamiliar faces, these pictures are more difficult to match for their identity than pictures of familiar(ized) faces (Bruce, 1982), even for simultaneously presented stimuli (Bruce et al., 1999; Megreya \& Burton, 2006; Jenkins et al., 2011). These observations have led a number of prominent researchers in the field to consider that unfamiliar FI ability of human adults is relatively poor and essentially based on low-level, "pictorial" cues of images (Hancock et al., 2000; Megreya \& Burton, 2006; Jenkins et al., 2011). In contrast, developmental studies in newborns and young infants often describe their ability to individuate unfamiliar faces as being impressive (e.g., Pascalis \& de Schonen, 1994; Turati et al., 2008), albeit using only simple tasks with very few stimuli and no quantitative measure of performance, thereby making it virtually impossible to compare their FI ability to that of older children and adults. This is an example of a clear contradiction that is not addressed in the field of human face recognition. Moreover, while behavioral studies point to a long developmental trajectory of FI (Carey, 1992; Mondloch et al., 2006; Hills \& Lewis, 2018; Mardo et al., 2018), it is still completely open to debate whether this development is due to general factors or specific to FI, and whether it essentially depends on genetic factors or experience (Mondloch et al., 2006; Crookes \& McKone, 2009; McKone et al., 2012; Mardo et al., 2018).

In the same vein, behavioral studies point to a large degree of inter-individual variability in the unfamiliar FI ability of neurotypical human adults (e.g., Bowles et al., 2009; Wilmer et al.,

3 The late Arthur Benton himself acknowledged that accuracy scores at his test were not diagnostic of prosopagnosia, but attributed that to the use of unfamiliar rather than familiar faces in his test (Benton, 1990). This does not appear to be the key contributing factor (see section 5.1.4 on Neural specificity and sources). 
2010; Bindemann et al., 2012; McCaffery et al., 2018; Stacchi et al., 2020), but the source of this variability remains unknown and may essentially reflect general task factors, as noted above. Hence, finding a significantly higher correlation of scores between monozygotic twins than dizygotic twins at the CFMT (Wilmer et al., 2010) should not be taken as evidence for a high genetic contribution specific to FI ability. Finally, the ability to individuate pictures of unfamiliar faces has been reported as being deficient in various neurological and neuropsychiatric pathologies, such as Alzheimer's disease (Lavallée et al., 2016), or Autism Spectrum Disorder (Weigelt et al., 2012), but these observations and their interpretations remain constantly questioned, essentially due to difficulties of comparing explicit behavioral performance across populations (Tang et al., 2015; Powell et al., 2019).

\subsection{The difficulties of event-related-potential measures of face individuation}

Implicit neural measures may be used to try to overcome the issues outlined above to advance our understanding of human FI. Recording electrical waves from the scalp, namely electroencephalography (EEG, since Berger, 1929), is a technique with high extendibility across populations tested. Standard EEG paradigms consist of presenting stimuli at relatively long, random intervals to isolate voltage changes over time, termed Event-Related Potentials (ERPs), after averaging across trials in the time-domain (Dawson, 1954; Luck, 2014). This approach, also applied to magnetoencephalography (MEG) to identify Event-Related Magnetic Fields (ERMF), can provide highly valuable information about the time-course of face recognition in general, including FI (for reviews: Rossion \& Jacques, 2011; Schweinberger \& Neumann, 2016).

However, we argue that this typical approach is limited in providing sensitive, reliable, and objective FI measures at the individual level. For instance, a number of studies have found that the face-sensitive, occipito-temporal N170 ERP is reduced following the immediate repetition of the same individual face (e.g., Heisz et al., 2006; Jacques et al., 2007; Caharel et al., 2009; Vizioli et al., 2010). Although the N170 itself is a sharp and readily identifiable face-selective deflection in 
the EEG signal, with reliable amplitude and latency within participants (Kaltwasser et al., 2014; see also Gaspar et al., 2011), the N170 face identity repetition effect remains relatively small at the group level (Rossion \& Jacques, 2011). Moreover, test-retest reliability of this FI effect is unknown, and it is virtually impossible to obtain at the single-subject level in a large majority of individuals, even when tested with a large number of trials (Jacques et al., 2007). A later ERP component, the N250, has been also used in similar repetition paradigms as a FI marker (i.e., the N250r, "r" for repetition effect; e.g., Schweinberger et al., 2002; Tanaka et al., 2006; Schweinberger \& Neumann, 2016 for review), but suffers from the same limitations. Additionally, it is sometimes difficult to identify at the individual level, and in contrast to the N170, the faceselectivity of the N250 time-window is unclear (Engst et al., 2006; Kaltwasser et al., 2014).

For these reasons, while EEG measures obtained during slow, jittered, stimulation can sometimes distinguish subgroups of good and poor face identity recognizers (Towler et al., 2012; Parkteny et al., 2015; Turano et al., 2016; Fisher et al., 2017) and are undoubtedly useful for characterizing the time-course of FI processes at the group-level ${ }^{4}$, they cannot serve as robust diagnostic markers at an individual level. It is especially difficult to objectively compare ERP components or time-windows of interest across developmental groups due to changes in latency, shape, and/or polarity (e.g., Taylor et al., 2004; Kuefner et al., 2009). In general, the weak sensitivity to FI and low reliability of such ERP components/time-windows also prevents their use as measures of immature or deficient FI processes during typical and atypical development (Vettori et al., 2019a).

\footnotetext{
${ }^{4}$ In recent years, measures of the time course of unfamiliar face individuation in EEG or MEG have also been reported, capturing differences between responses to individual faces across time and space using multivariate pattern analysis techniques (e.g., Nemrodov et al., 2016; Vida et al., 2017). However, since there is no reason to expect differences in absolute global EEG/MEG signals between different facial identities, these measures essentially capture, just above chance level, image-based low-level effects that are found usually very early (e.g., onset latency $<100 \mathrm{~ms}$ ) and inconsistently across studies.
} 


\subsection{The value of fast periodic visual stimulation}

A potential solution is to turn to an alternative stimulation and data analysis approach in EEG. This approach takes advantage of a periodically presented stimulus driving a periodic response of the brain's electrophysiological activity. Resultantly, the temporal frequency of the brain's response matches that of the stimulus presentation (e.g., a flickering light at $17 \mathrm{~Hz}$ in the initial study of Adrian \& Matthews, 1934). As Adrian himself noted, this stimulation mode provides high objectivity in identifying the response of interest: “...by making the field more or less uniform and lighting it with a flickering light, the nerve cells are forced to work in unison at the frequency of the flicker, and we can record their electrical activity through the skull up to frequencies of about 30 a second. This gives us a method of tracing the visual messages in the brain, for by means of the flicker rhythm they can be made easy to recognize." (Adrian, 1944, p.361). Regan (1966) subsequently named this approach "steady-state visual evoked potentials" (SSVEPs) and applied the Fourier Transform to express these digitally-captured periodic EEG responses (typically located over medial occipital channels) in the frequency-domain. Regan and others noted the substantial advantages of this EEG approach in terms of objectivity ("easy to recognize") and sensitivity (high signal-to-noise ratio, SNR) of the responses. Yet, although this stimulation approach preceded the report of the first ERPs (following the invention of the trial averaging method in EEG by Dawson, 1954), until recent years, SSVEPs remained essentially confined to studies of low-level sensory responses (i.e., following changes of stimulus luminance, contrast, and orientation) and their modulation by attentional factors (for reviews, see Regan, 1989; Norcia et al., 2015).

Over the last decade, this "frequency-tagging" or "fast periodic visual stimulation" (FPVS) approach $^{5}$ in human EEG has been extended to measure higher-level functions, and in particular

\footnotetext{
5 The term "SSVEP" is not used here because it refers to the type of response obtained rather than the approach, and is a loaded term, with different researchers having different views on what is and what is not a "SSVEP" (as opposed
} 
FI (Rossion \& Boremanse, 2011). The present paper focuses on a specific FPVS paradigm based on a periodic "oddball" sequence developed over the past few years (since Liu-Shuang et al., 2014), that aims to go beyond overt behavioral or ERP measures to investigate FI.

\section{Face individuation with an oddball FPVS paradigm and EEG}

\subsection{The oddball FPVS paradigm}

The current oddball FPVS design draws inspiration from several sources. First, so-called adaptation/habituation paradigms, in which responses to repeated visual stimuli are contrasted with responses to differing stimuli, have been used for a long time in human EEG research (Ritter \& Vaughan, 1969) in particular to describe visual discrimination responses (Simson et al., 1977). In the domain of face recognition, specifically, ERP repetition paradigms leading to adaptation/habituation effects have been used for decades, with various stimulation parameters (e.g., Barrett et al., 1988; Kovacs et al., 2006; Jacques et al., 2007; Schweinberger \& Neumann, 2016). Single neuron recordings to face stimulus repetition have also been described in the monkey superior temporal sulcus (Rolls et al., 1989; Li et al., 1993), and in human ventral occipito-temporal face-selective brain regions with functional magnetic resonance imaging (fMRI; Gauthier et al., 2000; Grill-Spector \& Malach, 2001). Second, a study by Rossion and Boremanse (2011) showed that FPVS and EEG, coupled with stimulus repetition, could be used successfully to study higherlevel processes such as FI, with high sensitivity in a short amount of time. In that study, a periodic 3.5 Hz EEG response was much larger for a stimulation sequence in which different facial identities were presented at every $3.5 \mathrm{~Hz}$ cycle compared to a sequence in which the same facial identity was repeated at that rate (see also Rossion et al., 2012; Alonso-Prieto et al., 2013). Third, these two methodological EEG approaches, adaptation/habituation and FPVS, have been previously

to a transient ERP; e.g., see Retter \& Rossion, 2016 for discussion). The term "frequency-tagging" is more appropriate, although often used in the context of spatially distinct stimuli flickering at different frequencies (Regan \& Heron, 1969; see Norcia et al., 2015) and this is why the term "FPVS" is preferred here. 
combined in oddball designs in a handful of studies in which periodic visual changes are introduced within a stream of periodic repeated stimuli (Braddick et al., 1986; Braddick et al., 2005; Heinrich et al., 2009; Hönegger et al., 2011). However, only simple grating stimuli and salient low-level stimulus changes (e.g., orientation) were used in these studies, and in some studies participants were required to explicitly attend to oddball stimuli (Heinrich et al., 2009; Hönegger et al., 2011) .

In the specific oddball FPVS-EEG paradigm reviewed here (Liu-Shuang et al., 2014; Figure 1), one facial identity is randomly selected from a large set of faces (usually $25-100$ individual exemplars, e.g. Figure 2). This "base" facial identity (A) is repeated at a constant rate $F \mathrm{~Hz}$, through contrast modulation (from 0 to $100 \%$ luminance contrast; either graded, e.g., sinusoidal, as in most studies so far, or abrupt, e.g., squarewave) over a stimulation sequence of about one minute. Throughout this sequence, "oddball" facial identities (i.e., those remaining in the original face set: identities B, C, D, E..., selected at random) are inserted among the base face presentations at regular intervals of every $n^{\text {th }}$ face (e.g., often 1/5). As a result, facial identity changes occur periodically at $F / n \mathrm{~Hz}$ (e.g., if $F=6$, and $n=5$, at $6 / 5 \mathrm{~Hz}=1.2 \mathrm{~Hz}$ ). Hence, in a design with a base frequency of $6 \mathrm{~Hz}$ and an oddball frequency of $1.2 \mathrm{~Hz}(6 / 5)$, the pattern of face identities is $A A A A E A A A A C A A A A F A A A A H A A \ldots$ (usually with a different base facial identity used in each stimulation sequence). Typically, observers complete an orthogonal task while viewing the image sequences (i.e., detecting brief and random color or shape changes of a central fixation cross overlaid on the image stream). Two to four sequences are usually presented for each experimental condition, leading to a total of only 2-4 min. testing duration per condition. See again Figure 1 for the full illustration of this paradigm.

Crucially, each the $F$ and $F / n$ frequency selectively "tags" the responses evoked at that rate in the EEG signal (see Figure 3A\&B). That is, $F \mathrm{~Hz}$ is the general visual stimulation frequency and $F / n \mathrm{~Hz}$ is the facial identity change frequency: the EEG responses to visual stimulation thus occur at $F$, and to facial identity change occur at $F / n$. Since $F / n$ is a proportion of $F$, if the identitychange response is not different from the responses to the repeated base facial identity, no $F / n$ 
response would be produced (e.g., this is the case in a well-known brain-damaged case of prosopagnosia: Liu-Shuang et al., 2016; see Section 5.1.3). For this reason, the identity-change response is a differential response, reflecting the difference in neural activation to oddball vs. base faces. Note that any systematic (i.e., periodic) deviation between the responses to the oddball faces $v s$. the responses to the base face could generate a response at $F / n$. Theoretically, this deviation could be an increase of response amplitude, a decrease and/or even a phase shift (i.e., a delay or acceleration of the response) introduced by the appearance of the different facial identity (see Section 6 below, on mechanisms).

In order to identify the frequency-tagged EEG responses, following minimal preprocessing steps, EEG data is transformed from the time-domain into the frequency-domain via Fourier analysis (Regan, 1966; this may be done rapidly with a standard Fast Fourier Transform (FFT)) (Figure 3C). 
A

\section{Stimulation sequence}

- 5 s sequence duration

- $\mathbf{5 1 2} \mathrm{Hz}$ sampling rate

B

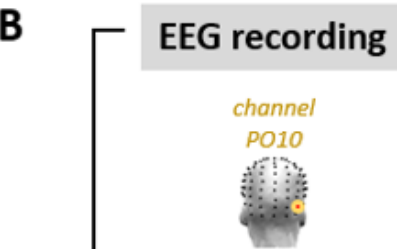

D

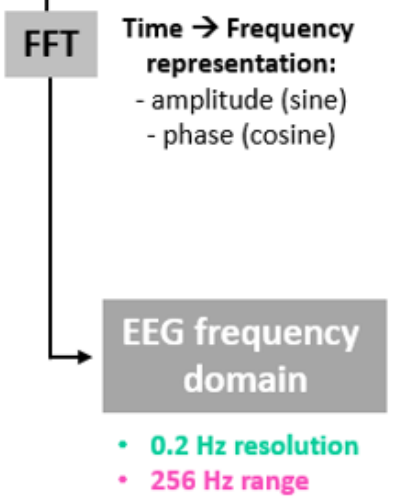

E

Multi-harmonic
combination
e.g., summation
with baseline subtraction
$0 \mathrm{~Hz}=$ target signal
with relative noise surround
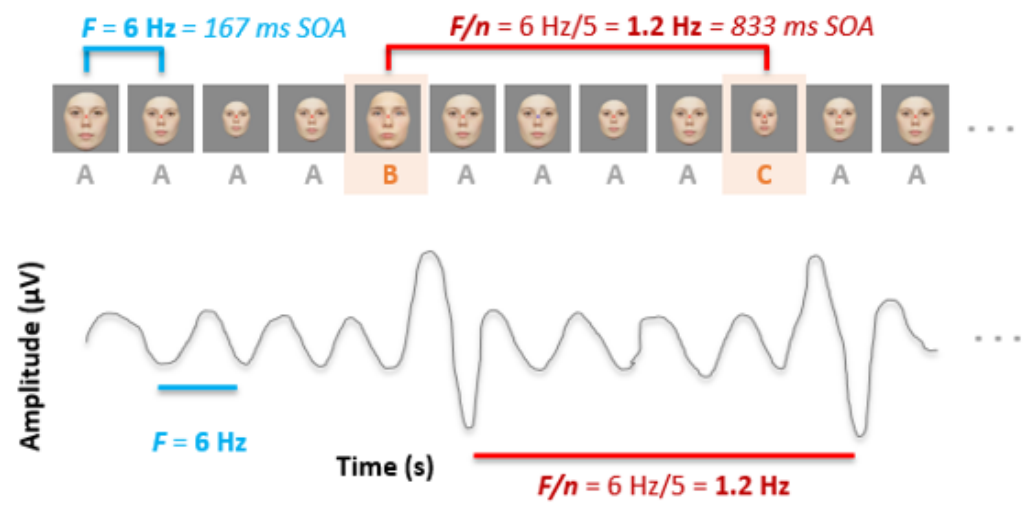

$\mathrm{F} / \mathrm{n} \mathrm{Hz}$

$2 \mathrm{~F} / \mathrm{n} \mathrm{Hz}$

$3 F / n \mathrm{~Hz}$

$4 F / n \mathrm{~Hz}$

$\mathrm{FHz}$

$6 \mathrm{~F} / \mathrm{n} \mathrm{Hz}$

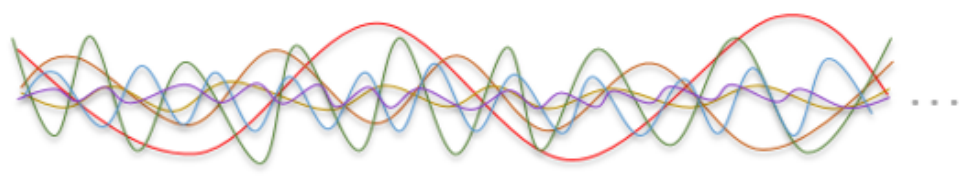

..
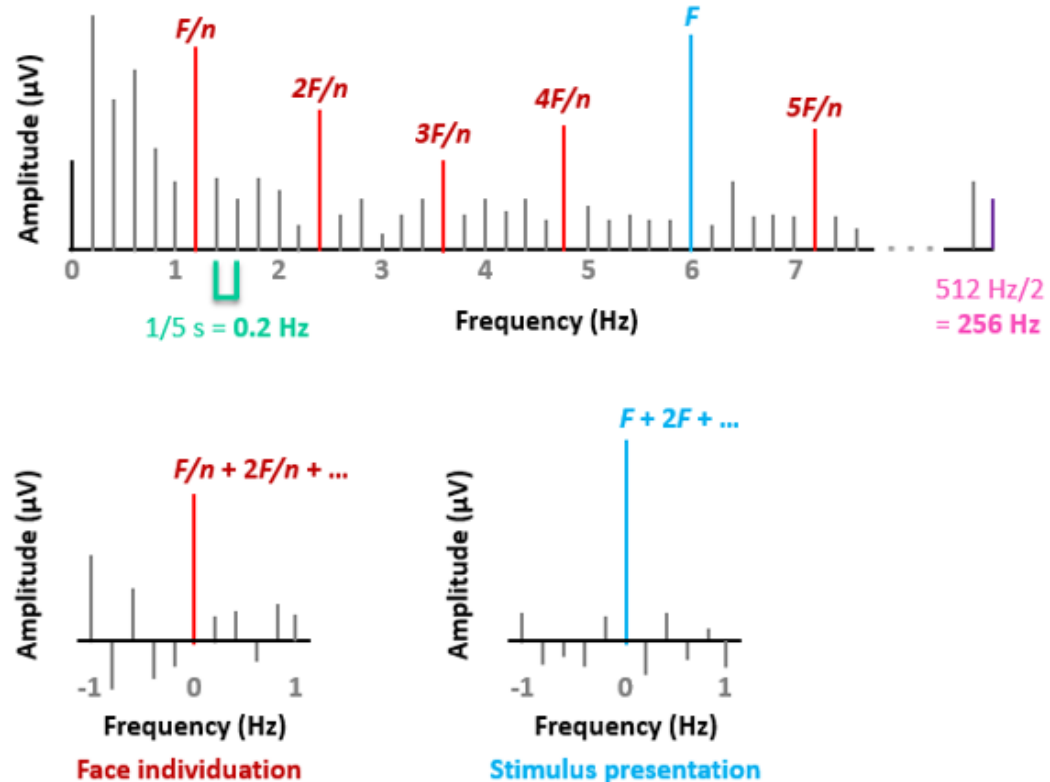

Figure 3. Basics of time to frequency domain representations for oddball FPVS with EEG.

A. The stimulation sequence, with face images presented at frequency $F$, and facial identity changes occurring every $n$ images, i.e., at frequency $F / n$. B. EEG recording. Stimulus-driven electrophysiological responses occur to each stimulus presentation, with distinctive responses to oddball as compared to repeated base faces. Neural responses are thus "tagged" at each the $F$ and $F / n$ stimulation frequencies (e.g., Fig. 6 of Liu-Shuang et al., 2014). C. The discretely sampled EEG signal is transformed into the frequency domain by means of a Fast Fourier Transform (FFT). The frequency-domain signal is constructed with a combination of sinewaves, each with its own amplitude and phase properties. A perfectly sinusoidal response would be captured in a single harmonic (at $1 \mathrm{~F}$, i.e., the fundamental frequency). More complex neural responses are described in the spread of the signal at not only $F$, but across its harmonic frequencies, that are uniquely periodic in terms of $F$ (i.e., occurring at the second harmonic frequency $=2 F$, third harmonic $=3 F$, etc.; see Box 1). The sum of these sinewaves represents the original signal. D. The resulting frequency amplitude spectrum (note that the corresponding frequency phase spectrum can similarly be 
computed). Its range is equivalent to $1 / 2$ of the sampling rate, and its frequency resolution is equivalent to the inverse of the sequence duration (e.g., $1 / 60 \mathrm{~s}=0.0166 \mathrm{~Hz}$; here, $1 / 5 \mathrm{~s}=0.2 \mathrm{~Hz}$ was used for display simplicity). E. Multi-harmonic response amplitudes can be combined (here, summed) along with a range of their surrounding frequency bin "noise" amplitudes (here, 5 bins below and above the target signal, defining a range of -1 to $1 \mathrm{~Hz}$ ), with a baseline correction (here, a subtraction, such that the noise level $=0 \mu \mathrm{V}$ ), in order to quantify/test the complete EEG response (Retter \& Rossion, 2016 see also Box 2). Note that harmonics of $F / n$ coinciding with $F$ are left out of the FI measure.

In the resulting frequency amplitude spectrum, peaks (i.e., stand-out amplitude values) are predicted at the relevant base $(F \mathrm{~Hz}$, e.g., $6 \mathrm{~Hz})$ and oddball $(F / n \mathrm{~Hz}$, e.g., $1.2 \mathrm{~Hz})$ frequencies, as well their harmonic frequencies (i.e., integer multiples of these fundamental frequencies; Figure 3D; see Figure 4 for actual data; see also Box 1). To quantify and evaluate these distributed multiharmonic responses, the fundamental and harmonic responses are combined: the amplitude of the combined "signal" is considered relative to the combined "noise" amplitude of nearby frequencies, indicative of baseline EEG activity (Figure 3E; see also Section 8.2.2).

Responses at the $F$ stimulus presentation frequency and its harmonic frequencies, reflecting the visual system's responses to the image stream, are a mixture of both low-level (e.g., periodic luminance changes that are not intrinsically characteristic of face stimuli, size changes) as well as higher-level processes (i.e., shape-related change and face-specific responses). This general $F \mathrm{~Hz}$ response is typically centered over medial occipital channels (Figure 5A), similar to SSVEP studies using low-level visual stimuli, or even for face stimuli when the exact same facial image is periodically repeated (e.g., Gruss et al., 2012; Wieser et al., 2012; Alonso-Prieto et al., 2013). By contrast, the response at the $F / n$ facial identity change frequency and its harmonic frequencies directly index FI and is typically located over lateral occipital and occipito-temporal channels, with a right hemisphere dominance (Figure 5B). Thus, throughout this review, we refer to responses at $F$ and its harmonics as general visual responses, and responses to $F / n$ and its specific harmonics as FI responses. 


\section{Box 1. Harmonic frequency responses}

The harmonic frequency responses observed at in the current paradigm are due to multiple factors. In general, harmonic EEG responses can be interpreted as the result of non-linearities of stimulus presentation and/or neural responses. That is, under the ideal conditions of perfectly sinusoidal stimulus presentation, a perfectly linear neural response (i.e., sinusoidal, amplifying or decreasing input without distortion) would be generated in the time domain, which would be represented by a single frequency in the frequency domain. This is certainly not the case here. By using complex stimuli (i.e., images of faces containing numerous pixels of varying luminance values) instead of a uniform light field, and due to technical limitations (i.e., monitor refresh rate and mode), the visual stimulation is not perfectly sinusoidal and inherently contains power at multiple harmonic frequencies. Moreover, brain processes at multiple levels of organization (synaptic transmission, single neuron firing, neural population responses, etc.) are to a large extent non-linear (e.g., Regan, 1989; Carandini \& Heeger, 2011; Zhu \& Rozell, 2013). These non-linearities in stimulus-factors and the brain's responses both predict multi-harmonic responses.

Empirically, the harmonic responses observed in the oddball FPVS paradigm do not appear to be driven by stimulus-factors: similar harmonic response amplitude distributions have been produced for (imperfect) sinewave $v s$. squarewave stimulus presentations (compare, e.g., Figure 4A (sinewave) to Yan et al., 2019 (squarewave); for different within-study comparisons, see: Retter et al, 2016: Dzhelyova et al., 2017). The FI harmonic responses may thus be considered mainly in light of the non-linear, complex neural responses elicited from the presentation of an oddball face in the time-domain (Figure 19; LiuShuang et al., 2014; Dzhelyova et al., 2014b; Yan et al., 2019). Crucially, such complex neural responses in the time domain predict complex harmonic amplitude distributions in the frequency domain. This is in line with complex signals being captured in Fourier analyses through the combination of sinewaves at multiple frequencies: in the case of periodic stimulus presentation, the only relevant frequencies are those periodic to the stimulation and its harmonics.

Importantly, the complexity of the EEG response is affected by the amount of processing time allowed, which is related to the stimulus presentation frequency, $F$. In the case of high frequencies of stimulation, interference occurs between consecutive neural responses that overlap in time (Keysers $\&$ Perrett, 2002), i.e., when the duration of the recorded neural response (e.g., about $400 \mathrm{~ms}$ for faces among visual objects) is longer than the duration of the stimulus presentation (i.e., $F>1 / 0.4 \mathrm{~s}=2.5 \mathrm{~Hz}$ for faces among objects; Retter \& Rossion, 2016). This neural interference between successive evoked responses suppresses the response amplitude and distorts latency, driving responses to follow an oversimplified, i.e., more sinusoidal, pattern (see Keysers et al., 2001; Retter et al., 2016; Retter et al., 2018). Thus, when $F$ is relatively high, the bulk of the response amplitude will typically be generated at the fundamental 
harmonic (i.e., $1 F$ ), with little response at the subsequent harmonic frequencies (e.g., see the moderately sinusoidal time-domain responses to fast stimulus presentation, $F=6 \mathrm{~Hz}$, in Figure 19A, which are represented in the frequency domain with a predominant first harmonic amplitude, in Figure 14A; for further examples across stimulus presentation rates: Alonso-Prieto et al., 2013; Retter \& Rossion, 216; Zhou et al., 2016; Bekhtereva et al., 2018 ; Retter et al., 2020). Indeed, at $15 \mathrm{~Hz}$, EEG responses to visual stimulus presentation recorded on the scalp can appear as nearly perfect $15 \mathrm{~Hz}$ sinusoids. Such sinusoidal responses are sometimes considered to be "true SSVEPs", i.e., as having reached a "steady-state". However, this is not a requirement for recording frequency-tagged responses (see again Footnote 2).

In contrast, slower stimulus presentation rates, with less neural interference, lead to more complex temporal responses, with rich harmonic frequency amplitude distributions. This is the case with the present FI responses at $F / n$ using the oddball FPVS paradigm, in which the $F / n$ is typically at a low rate (e.g., $1.2 \mathrm{~Hz}$; see Figure 4 for distributed harmonic response amplitudes; together with Figure 19 for the corresponding, complex time-domain responses). Again, these harmonic frequency responses (each defined by amplitude and phase) reflect the shape (deflections of voltage across time) of the neural response, the combination of which reconstructs the dynamics of the original neural response (see Figure 3C). To measure the complete neural response, these distributed harmonic responses may be summed (Retter \& Rossion, 2016; see Figure 3E; section 8).

\subsection{Large-scale group analysis}

Including the initial study published six years ago (Liu- Shuang et al., 2014), this paradigm has been used in 20 published studies to date, with a number of reports in preparation (see Table 1). One objective of this review is to provide deeper insight into the properties of FI as measured with the current paradigm. To this end, we included findings from yet unpublished studies from our group (the Face Categorization Lab) in our discussion.

\begin{tabular}{|l|l|l|}
\hline & Study & Topic \\
\hline 1 & Liu-Shuang et al., 2014, Neuropsychologia & $\begin{array}{l}\text { Original paradigm, effects of inversion and contrast } \\
\text { reversal }\end{array}$ \\
\hline 2 & Dzhelyova et al., 2014a, BMC* & Parametric effect of size variation \\
\hline 3 & Dzhelyova et al., 2014b, Journal of Vision & Effect of surface and shape cues \\
\hline 4 & Liu-Shuang et al., 2016, Neuropsychologia & Single-case of prosopagnosia (patient PS) \\
\hline 5 & Xu et al., 2017, JOCN & N=50, Correlation with CFMT \\
\hline 6 & Lochy et al., 2017, JOCN & Individuation of artificial objects \\
\hline 7 & Feuerriegel et al., 2018, Biological Psychology & Effects of expectation on the FI response \\
\hline 8 & Jonas et al., 2018, Cortex & Palinopsia (intracerebral electrical stimulation) \\
\hline
\end{tabular}




\begin{tabular}{|l|l|l|}
\hline 9 & Barry-Anwar et al., 2018, Neuropsychologia & FI to monkey faces in 6- and 9-month olds \\
\hline 10 & Hagen \& Tanaka 2019, Neuropsychologia & Bird experts \\
\hline 11 & Dwyer et al., 2019, Vision Research & Autism Spectrum Disorder (adults) \\
\hline 12 & Vettori et al., 2019, NeuroImage Clinical & Autism Spectrum Disorder (8-12 years old) \\
\hline 13 & Dzhelyova et al., 2019, Visual Cognition* & Test-retest reliability at 2 months interval \\
\hline 14 & Stacchi et al., 2019, NeuroImage & 6 months test-retest, variation of fixation position \\
\hline 15 & Yan et al., 2019, Neuropsychologia & Task-related effects \\
\hline 16 & Stacchi et al., 2019, J. Neuroscience & Link between foveation of features and FI \\
\hline 17 & Damon et al., 2020, NeuroImage & $\begin{array}{l}\text { FI to human and monkey faces, upright and } \\
\text { inverted, in adults }\end{array}$ \\
\hline 18 & Dzhelyova et al., 2020, Perception & Correlation with the BFRT \\
\hline 19 & Lochy et al., 2020, Developmental Science & Pre-schoolers, effect of inversion \\
\hline 20 & Verofsky et al., 2020, Biological Psychology & Comparison of unfamiliar and familiarized faces \\
\hline 21 & Jacques et al., submitted & Human intracerebral recordings \\
\hline 22 & Bottari et al., submitted & Congenital Deaf signers \\
\hline 23 & Hagen, Laguesse et al., in preparation*+ & Inversion training \\
\hline 24 & Retter et al., in preparation & Viewing time for face individuation \\
\hline 25 & Rossion et al., in preparation & Electrophysiological composite face effect \\
\hline 26 & Or, Liu-Shuang et al., in preparation* & Sensitivity to head orientation variations \\
\hline
\end{tabular}

Table 1. List of the 26 studies reviewed here, in which the oddball FPVS paradigm was used to measure FI. * Data included in the large-scale group analysis; + inverted face data included in the largescale group analysis. The dataset of the group analysis is provided in the form of pre-processed cropped sequences, before time-domain averaging and FFT (http://doi.org/10.4121/uuid:8e8cfaf2-ab00-45b2-90a0623fabf75ca9).

Moreover, we performed a large-scale group analysis on data compiled from seven different studies (total $\mathrm{N}=130 ; 79$ females, mean age $=21.58 \pm 2.21$ ) within the Face Categorization Lab using the oddball FPVS technique outlined above with high-density EEG recordings. Two of these studies are published and two are in preparation for publication: these studies are starred in Table 1; another three studies represent currently unpublished data: please see the Acknowledgements). A subset of 79 participants (48 females) also completed a condition with faces presented inverted, i.e., upside-down (the studies containing inverted face data used in this analysis are labeled with a plus sign in Table 1). The data were analyzed together for an integrated presentation of the results (see Supplementary Material for details; see section $\mathbf{8}$ for methods; dataset available here upon 
publication of the paper: http://doi.org/10.4121/uuid:8e8cfaf2-ab00-45b2-90a0-623fabf75ca9). Upfront, this large-scale group analysis provides a rich characterization of the FI response's amplitude distribution across harmonic frequencies (Figure 4), summed-harmonic amplitude quantification across the scalp (Figure 5; and occipito-temporal ROI: see Figures 7D \& 12A), and hemispheric lateralization (Figure 6), at both the group- and individual-participant levels.

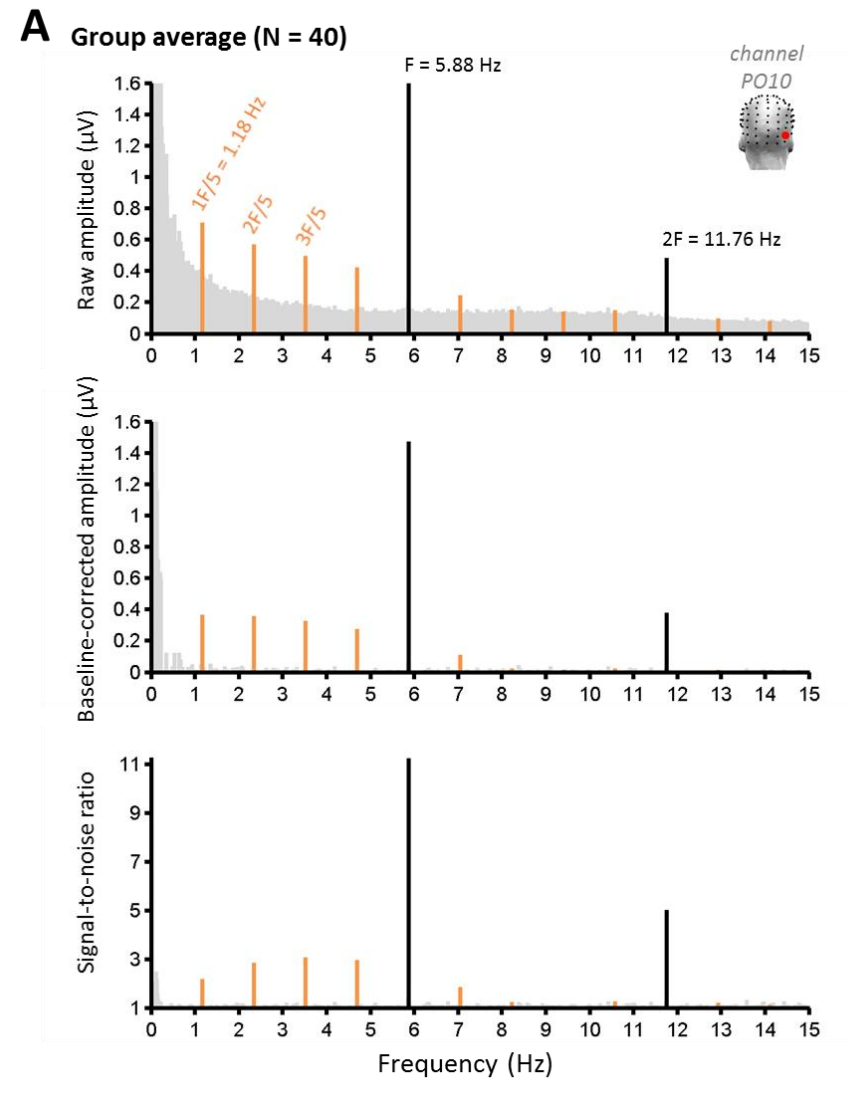

C

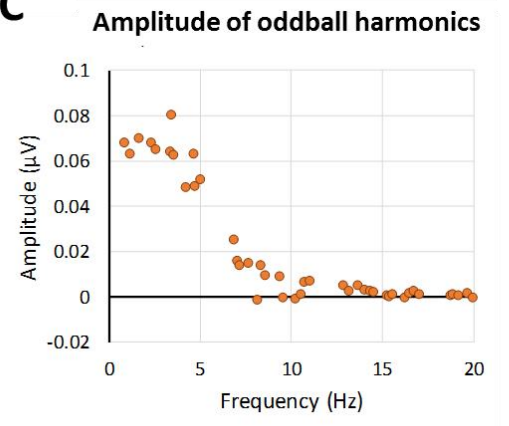

Distribution of harmonic amplitudes
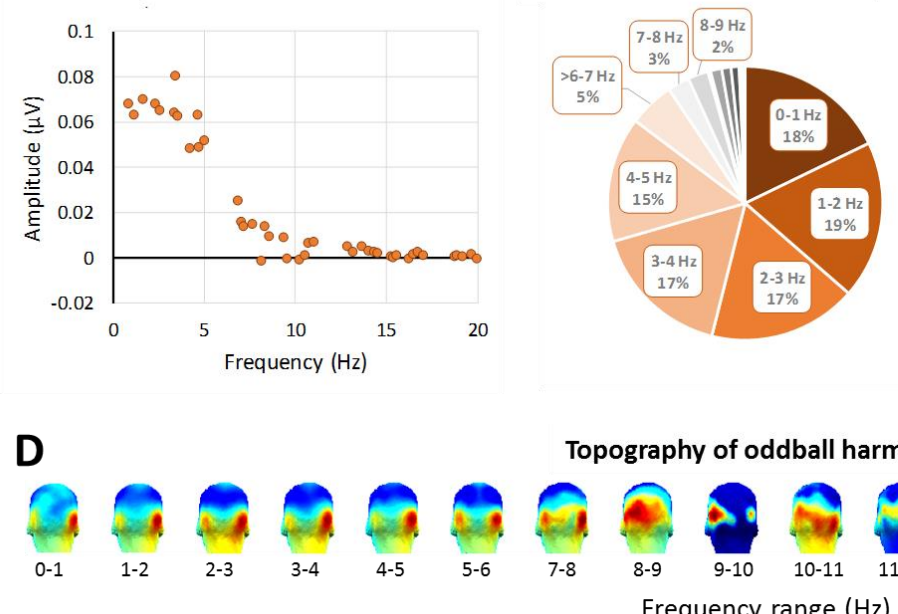

\begin{abstract}
Topography of oddball harmonics
\end{abstract}

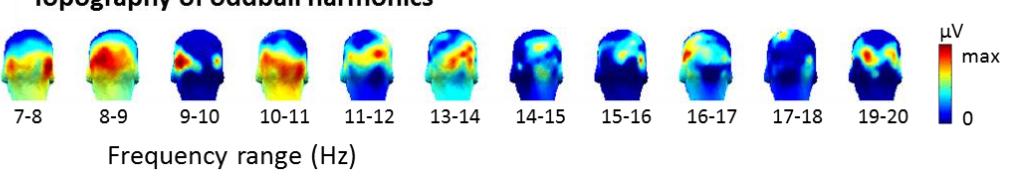

B Example individual participants

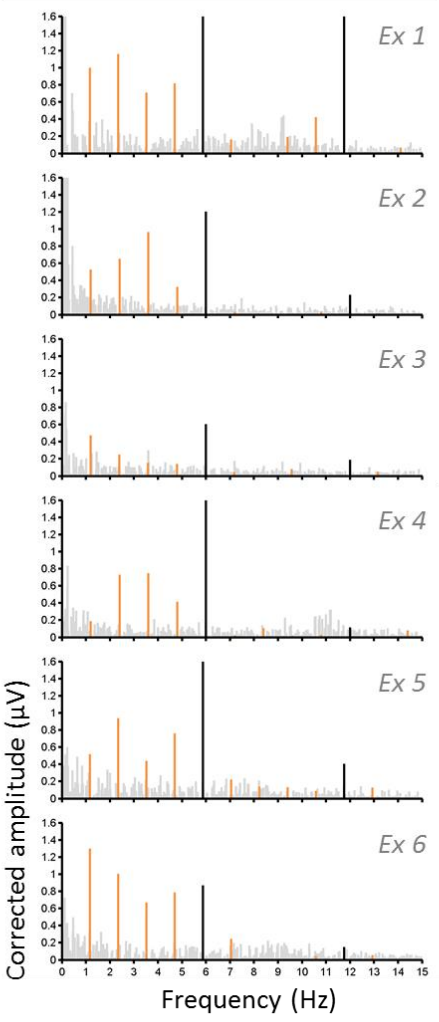

Compare oddball ratios

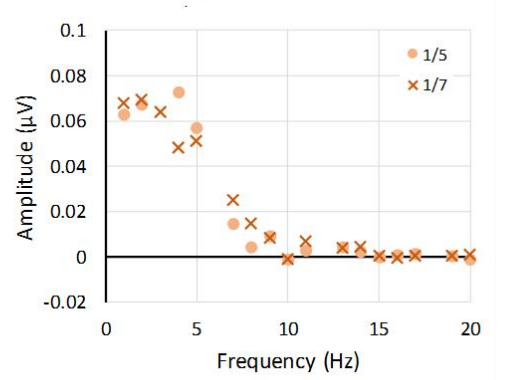

Figure 4. Harmonic frequency composition of the face individuation response (large-scale group analysis). 
A. Frequency spectra of occipito-temporal channel PO10 from an example subset of 40 participants who were shown stimulation sequences with a base rate $F=5.88 \mathrm{~Hz}$ (adjusting to a $100 \mathrm{~Hz}$ refresh rate monitor) and an oddball rate of $F / n=5.88 / 5=1.18 \mathrm{~Hz}$. Responses at these frequencies and their specific harmonics are highlighted in black $(F)$ and orange $(F / n)$, respectively. The top row displays the raw EEG amplitude values while the middle row displays the baseline-corrected amplitudes (i.e., with the average surrounding noise subtracted; see section 8). Unless specified otherwise, all further mentions of response amplitude refer to these corrected amplitudes. The bottom row displays the signal-to-noise ratio (SNR) of the EEG amplitude (see Methods), with SNR=1 being equivalent to noise level. B. Example amplitude spectra from 6 individual participants, shown to convey the amount of individual variability in the composition of the EEG spectrum and the robustness of the evoked periodic EEG responses. The different patterns of harmonics reflect the various shapes of the FI response in the time domain. C. Analysis of the harmonic frequency amplitude composition of the oddball frequency $F / n$ reflecting face individuation (based on scalp average amplitudes). The amplitude of the harmonic frequencies decreases sharply after $5 \mathrm{~Hz}$, such that the bulk of the FI response (86\%) can be accounted for by harmonics within the 1-5 Hz range. Note that the harmonic frequency amplitude distribution is identical across the $1 / 5(1.2 \mathrm{~Hz})$ and $1 / 7(0.86 \mathrm{~Hz})$ oddball ratios used here (far right panel). D. Topographical distribution of individual oddball harmonics within relevant frequency ranges, scaled according to their individual maxima. Note that a bilateral occipitotemporal topography persists up to $\sim 15 \mathrm{~Hz}$, after which the harmonic response amplitudes become negligible.

In regards to the group-level FI scalp topographies, across all of the EEG studies that have used this paradigm so far, the FI response consistently peaks in amplitude over bilateral occipitotemporal electrodes, more strongly over the right than the left hemisphere (for examples: Figures 5-9; but see also all studies reported in Table 1). In the present large-scale analysis, $43 \%$ of the overall scalp response is accounted for by relatively focal bilateral regions over the occipitotemporal cortex: $25 \%$ in the right hemisphere, and 18\% in the left hemisphere (Figure 5B\&C). Importantly, given the inherent uncertainty regarding the localization of the generators of electrophysiological responses recorded on the scalp (Nunez \& Srinivasan 2005; Luck 2014), this topographical pattern does not imply that the sources of the scalp response originate only or mainly from (right lateralized) ventral occipito-temporal regions thought to be responsible for FI from lesion studies of prosopagnosia (e.g., Meadows, 1974; Sergent \& Signoret, 1992; Bouvier \& Engel, 
2006; Barton, 2008). Yet, responses recorded on other electrode sites are not only weaker ${ }^{6}$ but also more broadly distributed, suggesting that they may merely reflect source diffusion on the scalp ("signal leakage"), dipolar scalp projections of neural activity in face-individuating regions, or an effect of the common average reference calculation in EEG (Luck, 2014). The neural sources of the FI response, as explored more appropriately with intracerebral EEG, are addressed in Section 5.1.4.

At the individual level, in $90 \%$ of individuals tested, the channel producing the maximal FI response amplitude is one of only 18 bilateral occipito-temporal channels (out of 128 channels, i.e. $14 \%$ of channels; for individual scalp topographies, see Figure 6B). Further, right lateralization is found in the majority of individuals: $73 \%$ of observers in the current group analysis, with a $\pm 8 \%$ standard deviation (Figure 6A). Overall, the FI response scalp topography is quite homogenous across individuals (e.g., Xu et al., 2017; Figure 6B; Figure 9), indicating that similar neurophysioanatomical networks are recruited to individuate faces with this paradigm.

${ }^{6}$ Technically, one could argue that, at the group level, the majority (i.e., 57\%) of the FI EEG response is recorded outside of the typical occipito-temporal ROIs, thus carrying "information" about individuation of faces. This forms the rationale for analyses carried out across the whole scalp with multivariate pattern analysis (MVPA) in EEG or MEG (e.g., Grootswagers et al., 2017), which could be applied to responses measured in the presently reviewed approach. However, these fluctuations of amplitude outside of the ROIs associated with the maximal response may be essentially due to signal leakage, which is problematic for MVPA analyses (Gohel et al., 2018). In line with this, in the present paradigm the responses of these "outside-ROIs" show little reliability (Figure 13). Accordingly, contrary to responses measured in the ROIs, there is no evidence that they could account for significant modulations of the FI response with experimental variables as tested across studies (Table 1). 
A

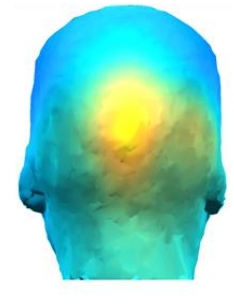

General visual response

B

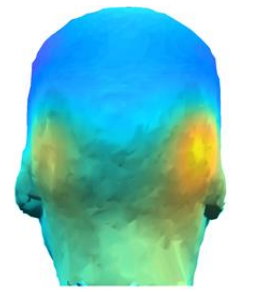

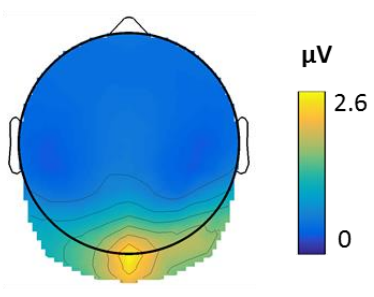

FI response

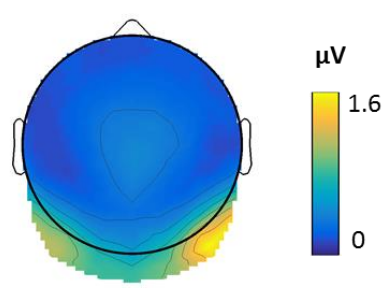

C Proportional distribution of FI response amplitude

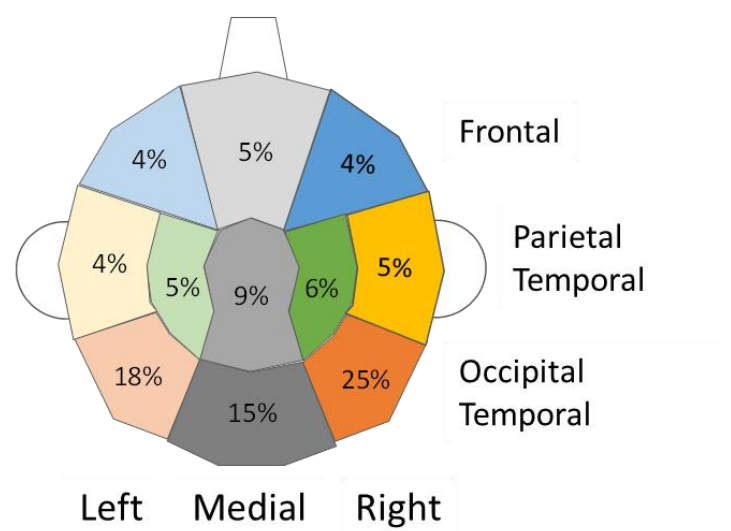

Figure 5. Scalp topographies of face individuation EEG responses (large-scale group analysis).

A. Group-averaged 3D and 2D scalp topographies of the general visual response (stimulus presentation rate $F$ and its summed harmonics), showing a peak over medial occipital channels (often channel Oz of the 1020 system). On average, responses within a medial occipital (MO) ROI reach $1.99 \mu \mathrm{V}( \pm 0.91 \mu \mathrm{V}$ standard deviation; see Supplementary Material). This scalp topography is typical of low-level "SSVEP" responses that are observed even for complex stimuli when the same image is repeated at a relatively high frequency. B. Group-averaged 3D and 2D scalp topographies of the face individuation response (oddball rate $F / n$ and its summed specific harmonics), displaying bilateral occipito-temporal activity with a right hemisphere lateralization (mean \pm standard deviation in the OT ROI used in further analysis $=1.19 \pm 0.81 \mu \mathrm{V}$; see Supplementary Material). C. Proportional distribution of the face individuation response across scalp regions (each subdivision contains 8 to 18 channels). The largest amount of response amplitude is concentrated in the right occipito-temporal region, and the bilateral occipito-temporal regions account for $43 \%$ of the total face individuation response. 
A

Histogram of face individuation response lateralisation
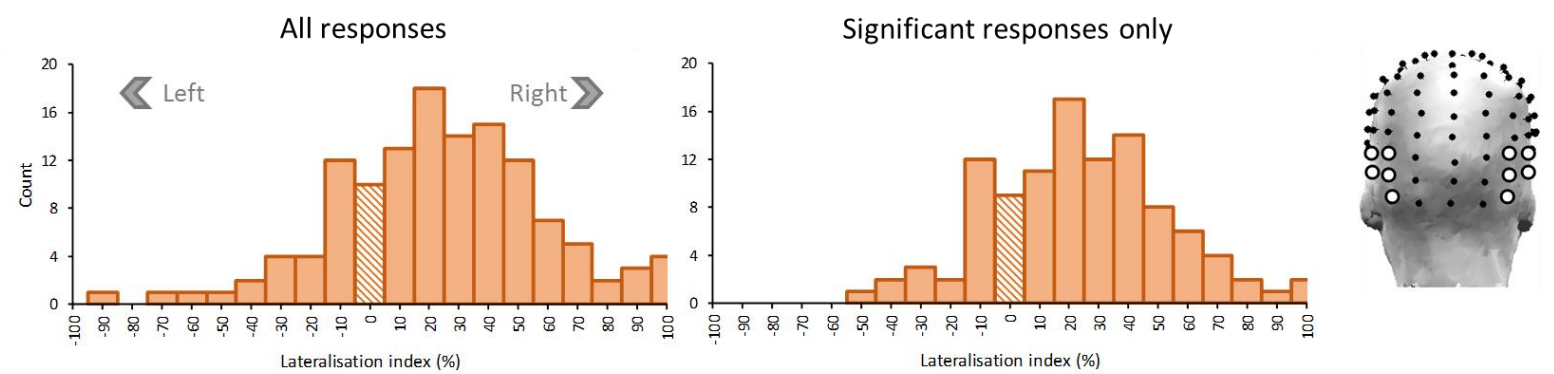

B Individual subject face individuation response topographies

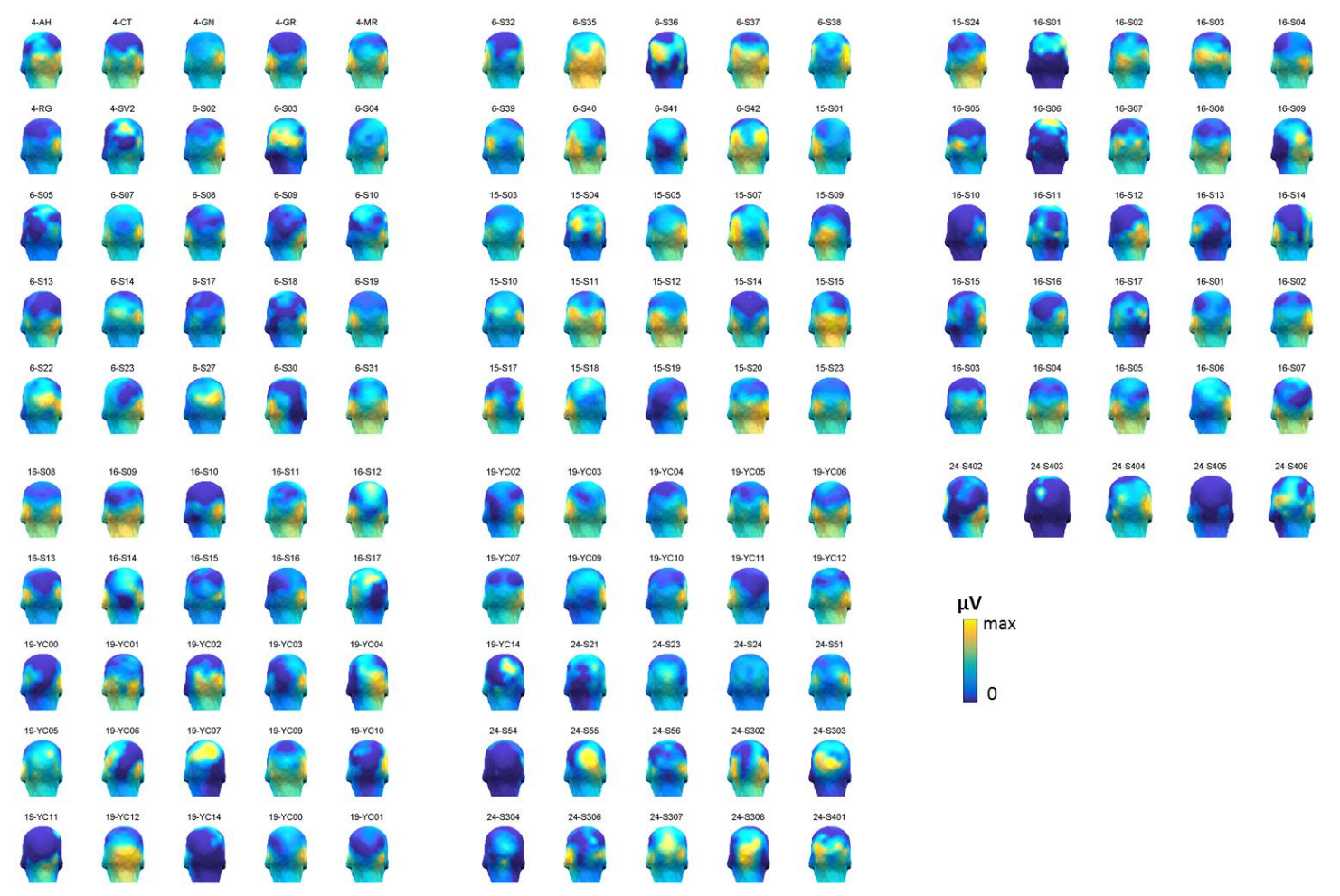

Figure 6. Lateralization of the face individuation response (large-scale group analysis).

A. Distribution of the lateralization index of the FI response ((Right-Left)/(Right+Left)x 100) across either all 130 participants (left) or only participants with significant responses at a conservative threshold (Z-score threshold=3.1; $\mathrm{N}=106)$. In both cases, the bulk of participants ( $70 \%)$ show right-lateralized responses, with an amplitude increase on average between 32\% (only significant responses) and 63\% (all responses) of hemispheric response difference. In contrast, left-lateralized responses are much rarer and show less strong hemispheric amplitude differences (18 to $23 \%$ on average). Note that all individuals here are right-handed, as assessed with an electronic version of the Edinburgh Handedness Inventory. The left and right channels composing each ROI are shown on the topography display on the right. B. 3D back view scalp topographies of the FI response across individual participants (scaled to individual maxima), demonstrating the consistency of the (right) lateralized occipito-temporal response. 
In the remainder of this review, we discuss the strengths and limitations of the oddball FPVS approach at the theoretical and methodological levels, including further results from the current large-scale group analysis, and draw implications for future research. Importantly, we present the oddball FPVS approach of measuring FI as an alternative or complementary measure to overt behavioral measures, rather than a measure that directly provides information about the neural basis and time-course of this function. However, specific recording and analysis procedures can be applied to also provide information about these aspects of FI, as will be illustrated in Sections 5.1.4 and 7.5.

\section{Advantages of the approach}

Is the FPVS oddball approach advantageous in terms of the criteria listed in Section 3.1, for providing a valid, reliable, objective, and sensitive measure of human FI? In the first section (Section 5.1), we will give an in-depth overview of the validity of the approach (i.e., does it truly measure FI?), which it is arguably the most important aspect and yet has the most ambiguous criterion, requiring an accumulation of evidence from multiple sources. We begin with a discussion of validity in view of the key characteristics of the $F / n$ FI response as an automatic, visual recognition process in neurotypical human adults (Section 5.1.1). We will also critique the ability of the paradigm to effectively exclude FI driven by low-level image-based cues, both from a theoretical (Section 5.1.2) and an empirical perspective. Thus, we will present evidence from lowlevel controls with inversion and contrast reversal, generalization across head orientation, as well as the significantly reduced FI responses in prosopagnosia (Section 5.1.3). We will also examine validity in regards to the specificity of neural FI signals and their potential neural sources (Section 5.1.4). Finally, we will comment on the validity of this oddball FPVS paradigm in terms of the neural mechanisms of frequency-tagged responses more generally (Section 5.1.5). The remaining criteria for a useful FI measure will be addressed in Section 5.2: objectivity (5.2.1), sensitivity (5.2.2) and reliability (5.2.3). 


\subsection{Is it a valid measure of face individuation?}

\subsubsection{Discrimination and generalization, automaticity, and time-constraints}

Can the electrophysiological response recorded in this paradigm be taken as a valid index of FI, i.e. does it truly measure this function? FI, as defined above, refers to the ability to discriminate, i.e., to provide different responses to different signals (here, different facial identities), while generalizing, i.e., providing the same (or a similar) response to different signals (the same individual face appearing under different viewing conditions). In the current paradigm (Figure 1), a $F / n$ (e.g., $1.2 \mathrm{~Hz}$ ) response can only emerge in the EEG spectrum if the individual facial identities appearing every $n^{\text {th }}$ stimuli generate a discriminable neural response, compared to a response evoked by the repeated facial identity at $F$ (e.g., $6 \mathrm{~Hz}$ ). As mentioned above, if no discrimination occurs at $F / n$, only a response at $F$ and its harmonics is expected, as is, for example, the case of the prosopagnosic patient PS (Liu-Shuang et al., 2016). The paradigm also measures generalization because the response generated by each instance of facial identity change has to consistently differ (i.e., be larger, smaller, or qualitatively different, see Section 6 on mechanisms) from the response to the repeated facial identity (Figure 3). In this way, the paradigm can be said to truly capture a neural categorization/recognition response, as defined at the beginning of the present review.

The FI response reflects automaticity as well, largely because FI is measured implicitly, i.e., in the absence of an explicit task related to face individuation (participants usually perform an orthogonal fixation cross task, as described in Section 4). Additionally, the fast presentation rate (e.g., $F=6 \mathrm{~Hz}$ equals an $\mathrm{SOA}=167 \mathrm{~ms}$ ) only allows for a single fixation per face presentation, forcing the system to individuate faces under tight temporal constraints and thereby preventing unnatural, feature-by-feature analysis strategies to discriminate the facial identities. 


\subsubsection{A high-level FI response despite identical base face images?}

Since the typical FPVS FI paradigm is based on repeating the exact same picture of an unfamiliar base facial identity, in contrast to different, unfamiliar oddball facial identities, mere image-based processing at the level of the retina, the lateral geniculate nucleus, or the early visual cortices could potentially contribute to the $F / n$ response recorded on the scalp. While the processes performed in these structures are critical for face individuation, the goal of the research is to isolate higher-level processes, ideally specific processes, involved in this function. Several aspects of the present paradigm minimize the influence of low-level image cues to the neural FI response.

First, typically all face images (both base and oddball) vary randomly and substantially in size for each presentation (generally between $80-120 \%$ of the original size, i.e., about $\sim 5 \times 3-\sim 8 \times 5$ degrees of visual angle; Figure 1). Hence, the paradigm is not strictly an oddball paradigm (where the exact same stimulus is repeated), and is sometimes described as an "oddball-like" paradigm. This stimulus size change leads to large fluctuations of pixel intensities at every stimulus presentation that are orthogonal to the facial identity changes. Hence, a cell in the retina or the primary visual cortex with a limited receptive field is stimulated with very different inputs at every base stimulus presentation, and is therefore "blind" to the identity change at the oddball rate. Empirically, this manipulation effectively decreases the FI response by about $23 \%$ for a $10 \%$ size variation (e.g. 95-105\%), with no further decrease for a $20 \%$ size variation (Dzhelyova \& Rossion, 2014a). A balance needs to be found between minimizing pixel overlap and reducing the sensitivity of the paradigm to capture FI. For example, larger size variations of $80 \%$ reduce response amplitude by half, but probably affected both low-level and higher-level processes (Dzhelyova \& Rossion, 2014a).

Second, the paradigm requires the repeated base facial identity (e.g., face A) to be individuated from multiple different facial identities (i.e., face B, face C, face D, etc.; Figures $1 \&$ 2). Hence, the neural discrimination response is based on different cues at every oddball presentation, and cannot be due to the specific features differentiating only two individual faces. 
Of course, if, by chance, the randomly selected base face differs from all other face identities in terms of global luminance level or contrast, these cues will contribute to the FI response. However, a systematic contribution of these cues is minimized by averaging data recorded across several stimulation sequences with different base faces, and also varying the selection of base faces across observers. Note that even when these cues are available for individuating faces, there is no reason to expect that a neurotypical human adult, who is an expert at FI, would predominantly rely on such cues rather than on higher level face-specific processes (Sergent, 1989).

Finally, the FI response with this paradigm has been obtained with various stimulus sets, such that it is not tied to the repetition of certain image-specific physical features (e.g., custom inlab face set: Liu-Shuang et al., 2014; human faces from the PrimFace database: Damon et al., 2019; Max Planck 3D scanned faces: Or et al., in preparation).

\subsubsection{A high-level FI response: empirical evidence}

In the following subsections, empirical evidence demonstrating the effectiveness of this paradigm to measure high-level FI is reviewed and explored through the current group analysis (see Section 4.2). Specifically, we address the impact of inversion and contrast negation, the generalization of FI across head views, and the (lack of a) prosopagnosic FI response.

\section{Contrast negation \& inversion}

One approach to demonstrate that the FI response reflects high-level processes is to preserve physical differences between the stimuli but show that the FI response is nevertheless severely deteriorated (a complimentary approach to that in which physical differences between stimuli are varied and the FI response is preserved, as described in the next section).

Contrast negation, whereby the luminance values of an image are flipped (i.e., black pixels become white and vice versa), is an image manipulation that preserves global contrast but significantly worsens the ability of neurotypical adults to perceive facial identity compared to other object categories (Bruce \& Langton, 1994; Russell et al., 2006). When comparing sequences 
containing greyscale and contrast negated face stimuli, even though physical differences between faces remain identical, the FI response amplitude to contrast negated faces is substantially decreased (Liu-Shuang et al., 2014). This observation supports the involvement of high-level processes in the FI responses. A drawback of contrast negation is the exclusion of relevant color information. Also, contrast-reversed faces typically have a lower overall luminance than normal faces, biasing direct comparison between the two types of stimuli. To overcome this issue, mean luminance could be equalized between positive and negative contrast faces (see Liu-Shuang et al., 2015 for a discussion of this issue).

By comparison, picture-plane inversion (i.e., turning an image upside-down either by mirroring the image along the midline or rotating by $180^{\circ}$ ) retains the natural appearance of a face and reflects a change of appearance that can occur in the real world, albeit very rarely. This image manipulation is extremely useful to exclude the contribution of low-level visual cues to the FI response. Indeed, even if low-level cues are not fully preserved by stimulus inversion, the physical difference between different individual faces is strictly preserved across inversion. Despite this, inverting image orientation substantially deteriorates the ability of neurotypical adults (who have developed expertise at individuating upright faces only) to recognize facial identities. This "face inversion effect" has been demonstrated across a wide variety of experimental paradigms and stimulation conditions, both for familiar and unfamiliar faces (Hochberg \& Galper, 1967; Yin, 1969; Freire et al., 2000; Collishaw \& Hole, 2000; for review see Rossion, 2008).

Here we summarize the effect of inversion on the FI response in the oddball FPVS paradigm with our large-scale group analysis (79 neurotypical adult participants; see Supplementary Material for details). When faces are inverted, the EEG FI response amplitude reduces to only $47 \%$ of the response to upright faces (Figure 7; see also Damon et al., 2020). Critically, this does not mean that $47 \%$ of neural FI response is elicited by low-level visual cues (a common misinterpretation)! Indeed, inverted faces are structured stimuli that also activate high-level visual regions of the human brain, including face-selective areas of the fusiform gyrus (Haxby et al., 
1999) or non-face high-level visual brain regions (Rosenthal et al., 2017), and these regions may also contribute to the inverted FI response (see Section 5.1.4 on the neural basis). Nevertheless, these findings show that more than half (i.e., 53\%) of the FI EEG response cannot be attributed to objective physical differences between images. Compared to the typical size of inversion effects reported in behavioral studies (a drop of around 20\% in accuracy, see Rossion, 2008), the effect observed in the present paradigm is particularly large, potentially thanks to the fast presentation rate, which prevents detailed exploration of local features (see Section 5.1.1.). Interestingly, when removing the common FI response to upright and inverted faces, the right hemispheric lateralization of OT regions increases (i.e., $29 \%$ for right OT vs. $15 \%$ for left OT as compared to 25\% vs. $18 \%$ as shown in Figure 5).

Another interesting observation is that the (Pearson) correlation of FI amplitude across individuals $(\mathrm{N}=79)$ between upright and inverted faces, while being highly significant $(\mathrm{p}<0.001)$, is relatively modest $(\mathrm{r}=0.55))$. Given that the test-retest reliability of the measure for upright faces with 4 sequences is very high (section 5.2.3), this indicates that a large part $\left(1-r^{2}\right.$, i.e. $\left.70 \%\right)$ of the variability in response amplitude between individuals is not accounted for by low-level cues. In comparison, correlations observed between performance for upright and inverted faces in behavioral face matching tests such as the GFMT are significantly higher (Megreya \& Burton, 2006), probably due to the contribution of general cognitive factors in these tasks. 

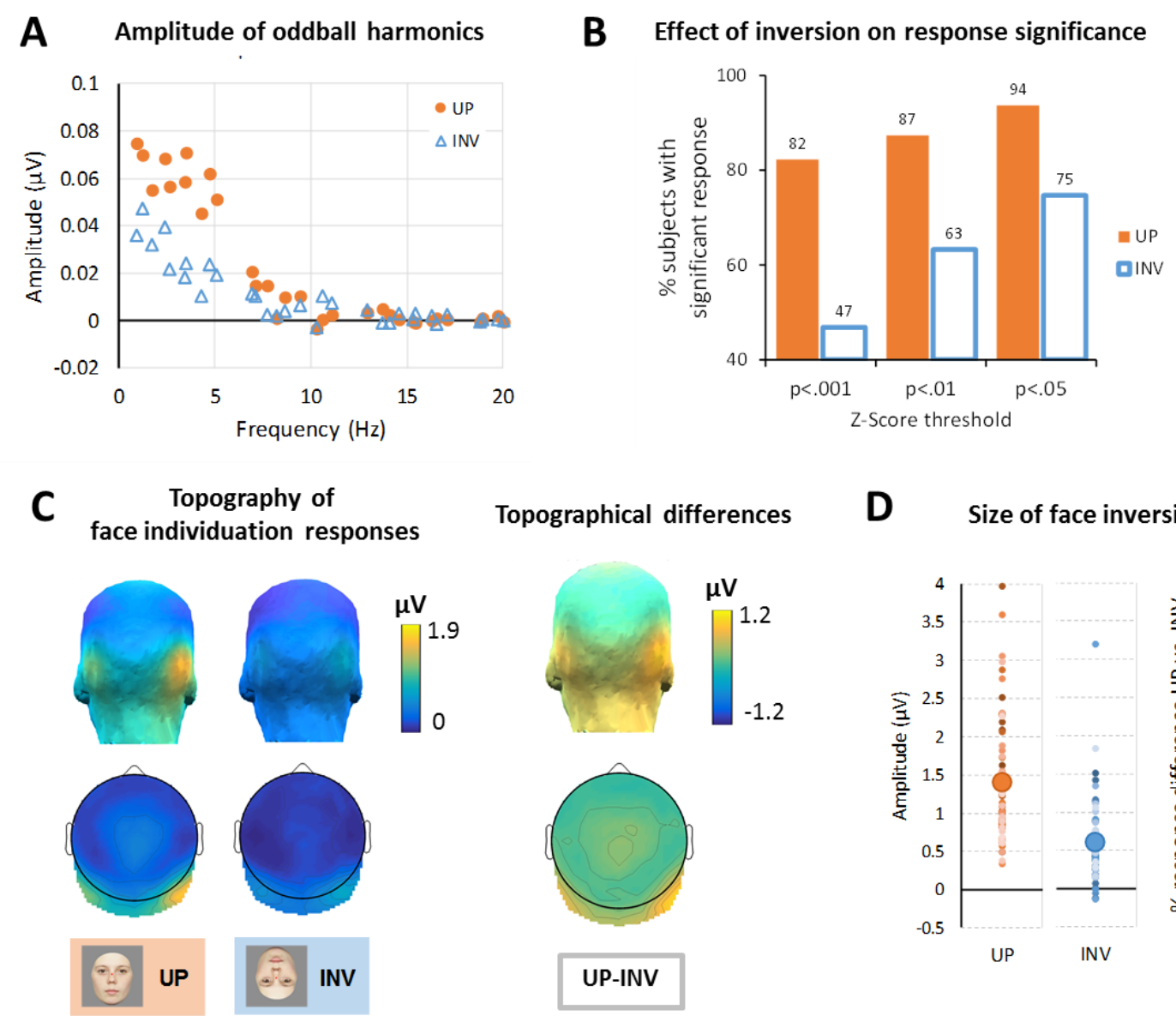

D Size of face inversion effect

Figure 7. Effect of picture-plane inversion on the face individuation response (large-scale group analysis).

A. The distribution of amplitude at oddball harmonic frequencies is similar for upright and inverted faces, but inversion significantly reduces the amplitude of harmonic frequency amplitudes below $10 \mathrm{~Hz}$. These multi-harmonic responses were summed for the following panels (see Section 4.1). B. Relative to the upright condition, inversion also decreases the proportion of participants with significant FI responses by about 20$30 \%$. C. Topographical analysis of the FI response for upright and inverted faces. Left: 3D \& 2D scalp topographies scaled to the maximum amplitude in the upright condition, demonstrating a substantial decrease in response amplitude. Right: Difference between scalp topographies for upright $v s$. inverted faces, showing that inversion mostly attenuates responses over the right occipito-temporal channels. D. Left: distribution of individual subject FI responses for upright and inverted faces (only subjects with significant responses in the upright condition at a threshold of $Z>3.1$ are included). Mean values are indicated by the large dot. Right: distribution of the percentage of response decrease for inverted faces relative to upright faces ((UP-INV)/UP). On average, inversion decreases FI EEG responses over the right occipito-temporal cortex by $\sim 53 \%$. 
Recent studies have shown that the FI inversion effect is due to knowledge of stimulus orientation derived from experience. In a developmental study, 5-year-old children had significant FI responses but showed only a small amplitude reduction with inversion (11\%; Lochy et al., 2020). In contrast, 8 to 12-year-olds tested with the same stimuli already show a much larger, almost adultlike face inversion effect (Vettori et al., 2019b; see Section 7.4 on development below). In a study with human adults, Damon et al. (2020) found that the FI response was substantially reduced following inversion for human faces but not for unexperienced monkey faces. Finally, observers who were intensively trained to individuate inverted faces over two weeks showed a significant increase of the response for inverted faces (reducing the effect of inversion by about half; Hagen, Laguesse et al., in preparation), in line with previous behavioral evidence (Laguesse et al., 2012). Taken together, these results support the view that the face inversion effect is essentially due to experience, as opposed to a pre-disposed (i.e., inborn) sensitivity of high-level visual regions to differences between upright facial patterns.

\section{Generalization across head view variations}

Another way to test the high-level nature of the FI response in this paradigm is to use pictures of the same unfamiliar facial identity taken under different viewing conditions. For instance, although the low-level visual input is quite different between a frontal and a side view of an unfamiliar face, the FI response should generalize across these view variations even for unfamiliar faces (Figure 8). Damon and colleagues (2020) presented oddball FPVS sequences containing human faces that varied in head pose (e.g., tilted down, looking upward, to the side, etc.). Critically, head pose was manipulated orthogonally to identity change: while facial identity changes were introduced at regular intervals, pose varied randomly at every stimulus presentation. Hence, a FI response could only arise if the system was both able to generalize across the variable poses of base faces (i.e., to consider them as being of the same individual) and to detect the identity changes despite unrelated head pose changes. The results of this study see also Bottari et al., submitted for changes of facial expressions at every base rate cycle) not only show a robust neural 
FI response for human faces that validates the involvement of high-level processes in this paradigm, but also underline the specificity of such image generalization to human faces, as FI responses were absent for monkey faces presented under the same conditions.

To more systematically examine the extent to which the neural FI responses generalize across head view variation, multiple amounts of head view variation, ranging from $0^{\circ}$ (no change, all full-front faces) to $\pm 90^{\circ}$ (faces could appear at any view between the left and right profile), were tested in $5^{\circ}$ increments in a recent study (Figure 8; Or et al., in preparation). Importantly, here too facial identity change occurred independently from view change. Results showed that FI responses remained significant for all viewpoint variation conditions, again demonstrating that the $F / n$ FI response goes well beyond simple low-level image discrimination. There was, however, a linear decrease of the response over the right occipito-temporal cortex with increasing viewpoint variation (Figure 8), indicating that this response is viewpoint-dependent to some extent. Note that this result does not imply the existence of viewpoint-dependent representations of unfamiliar face identities (i.e., neurons coding for facial identity only under specific views), as sometimes interpreted ${ }^{7}$. A more parsimonious explanation is that the same population of neurons carries out FI across various change in head views, but is less successful at associating identities when fewer cues match between the unfamiliar face exemplars.

\footnotetext{
${ }^{7}$ This interpretation of viewpoint-dependent representations of unfamiliar face identities comes essentially from nonbiologically plausible computational models of vision (e.g., Chung et al., 2006; Abudarham \& Yovel, 2020) as well as from monkey physiology studies, with face-selective neurons in the monkey superior temporal sulcus (STS) often responding to specific views of faces (Perrett et al., 1988; Freiwald \& Tsao, 2010). However, these neurons may have nothing to do with a coding of facial identity - they may instead code for head orientation (in relation to gaze direction), a much more important cue for social interactions in macaques (Emery et al., 1997; see Rossion \& Taubert, 2019).
} 


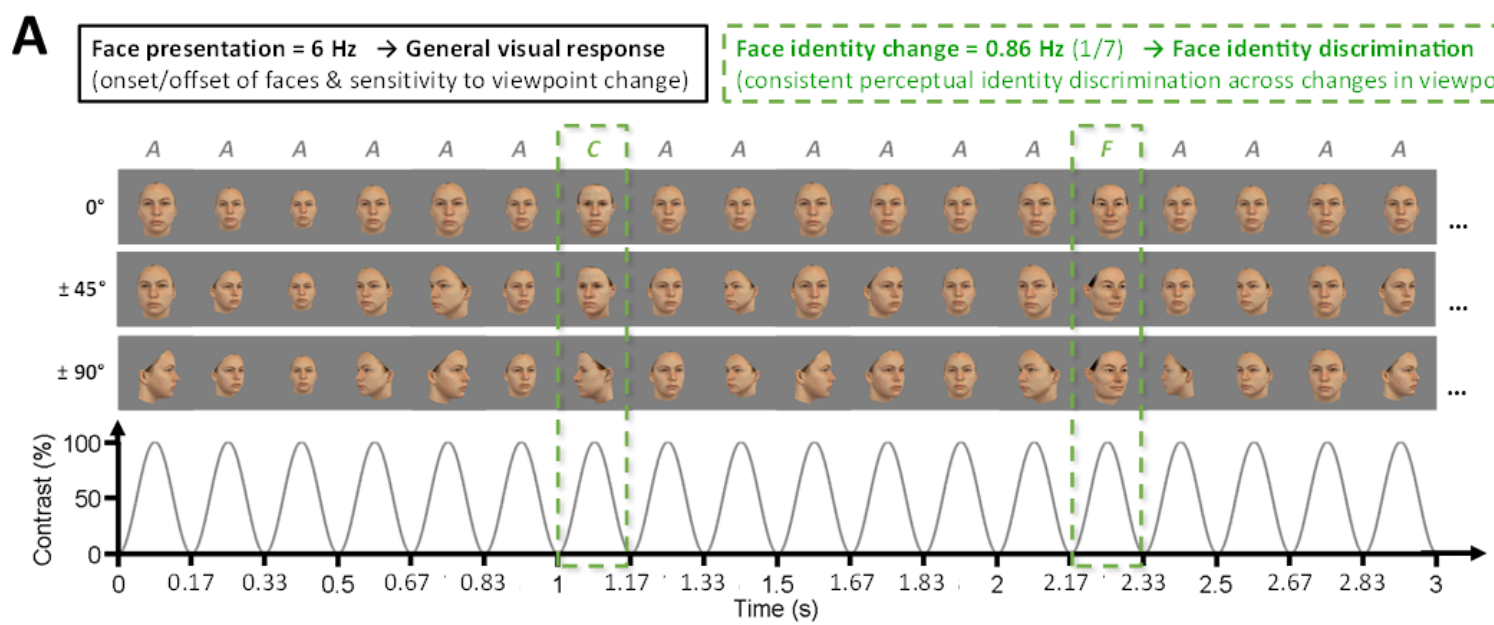

B Summed harmonics as a function of viewpoint variations

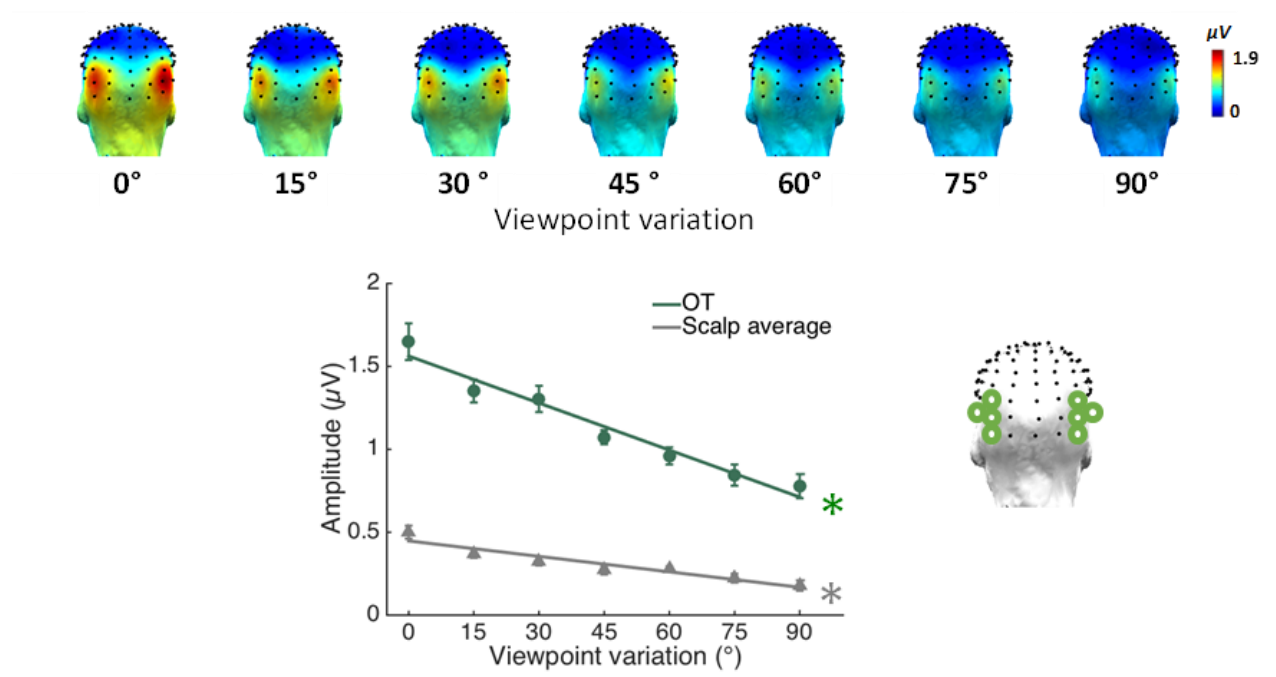

Figure 8. Generalization of the face individuation response across changes in head views (from Or et al., in preparation).

A. Stimuli were a set of 11 female facial identities from the MPI database of 3D scanned faces. For each face, 37 head views were used: $-90^{\circ}$ (left profile) to $90^{\circ}$ (right profile) in steps of $5^{\circ}$. In the experimental paradigm, face stimuli were shown at a rapid $6 \mathrm{~Hz}$ rate (i.e., 6 images/s) through sinusoidal contrast modulation, and varied randomly in size at each presentation. A randomly-chosen base facial identity (A) was repeated throughout the sequence, with different facial identities $(C, F \ldots)$ inserted as every 7 th face $(0.86 \mathrm{~Hz})$. Example sequences from three view conditions $\left(0^{\circ}, \pm 45^{\circ}, \pm 90^{\circ}\right)$ are shown here $(7$ conditions in the actual experiment: $0^{\circ}, \pm 15^{\circ}, \pm 30^{\circ}, \pm 45^{\circ}, \pm 60^{\circ}, \pm 75^{\circ}, \pm 95^{\circ}$ ). In the $0^{\circ}$ condition, all face stimuli were shown at a full-front view without head view change. In the $\pm 45^{\circ}$ and $\pm 90^{\circ}$ conditions, each face stimulus varied randomly within the range of $-45^{\circ}$ to $+45^{\circ}$, and $-90^{\circ}$ and $+90^{\circ}$, respectively. Periodic EEG responses at the stimulus presentation frequency $(F=6 \mathrm{~Hz})$ and its harmonics reflect responses to the onset and offset of face stimuli, whereas responses at the face identity change frequency $(F / n=0.86 \mathrm{~Hz})$ and harmonics specifically reflect FI across head view variations. B. The effect of head view on the FI response. Top panel: Observer-averaged scalp topographies showing response amplitude reduction with increasing view 
variation. Note that the spatial distribution of responses remained consistent. Bottom panel: Observeraveraged responses for the occipito-temporal (OT) ROI and for the scalp average. Channels composing the OT ROI are shown on the right. The error bars denote \pm 1 normalized SEM. Note that some error bars are too small to be visible. The asterisks reflect significant response reductions with head view variation based on linear regression analyses.

\section{Prosopagnosia}

The involvement of high-level process in this paradigm can also be validated with classical cases of prosopagnosia, i.e., patients who can no longer recognize facial identity following brain damage, despite intact object recognition skills and sufficiently preserved low-level visual processes (Bodamer, 1947; see Rossion, 2018 for other criteria of prosopagnosia). We tested the present paradigm on the patient PS, a well-known and extensively documented case of prosopagnosia (more than 30 scientific publications, from Rossion et al., 2003 to Gao et al., 2019). Strikingly, even when averaging 4 one-minute stimulation sequences, PS showed no significant FI response, contrary to all her age-matched control participants (Figure 9; Liu-Shuang et al., 2016; see also Gao et al., 2019 for a fMRI version of the paradigm tested with PS). Importantly, the absence of a FI response in the prosopagnosic patient PS cannot be due to a lack of sensitivity to rapidly presented stimuli, since she shows a large and significant response to image presentation at $F(=6 \mathrm{~Hz})$. Furthermore, when tested in another paradigm measuring the categorization of faces among non-face objects at the same stimulation frequencies as the FI paradigm, PS did exhibit a robust oddball response (Liu-Shuang et al., 2016).

It is particularly interesting to note that patient PS is able to match size-varying images of unfamiliar faces well above chance-level in behavioral studies (and in fact performing much better than macaques or other non-human species in such tasks; e.g., Rossion et al., 2003; Busigny et al., 2010). One important factor to consider is that the long presentation durations (most of the time until the patient provided a response) of the face stimuli in the behavioral studies allows her to slowly analyze the individual faces on a feature-by-feature basis in order to discriminate them (as indicated by her eye movement exploration on faces during a facial identity recognition task, Orban 
de Xivry et al., 2008). However, this is simply impossible to do in the oddball FPVS paradigm, which is temporally constraining (i.e., one fixation/face), without putting the patient under stress to perform an explicit task. Interestingly, when PS was presented with the $6 \mathrm{~Hz}$ stimulation sequence outside of EEG recordings and informally asked to tell experimenters how many facial identities she perceived, she appeared unable to detect the rapid (periodic) changes of facial identity and reported only seeing one identity throughout the one-minute stimulation. These observations further suggest that the present paradigm could prove highly valuable towards the diagnosis of FI impairments in prosopagnosia.
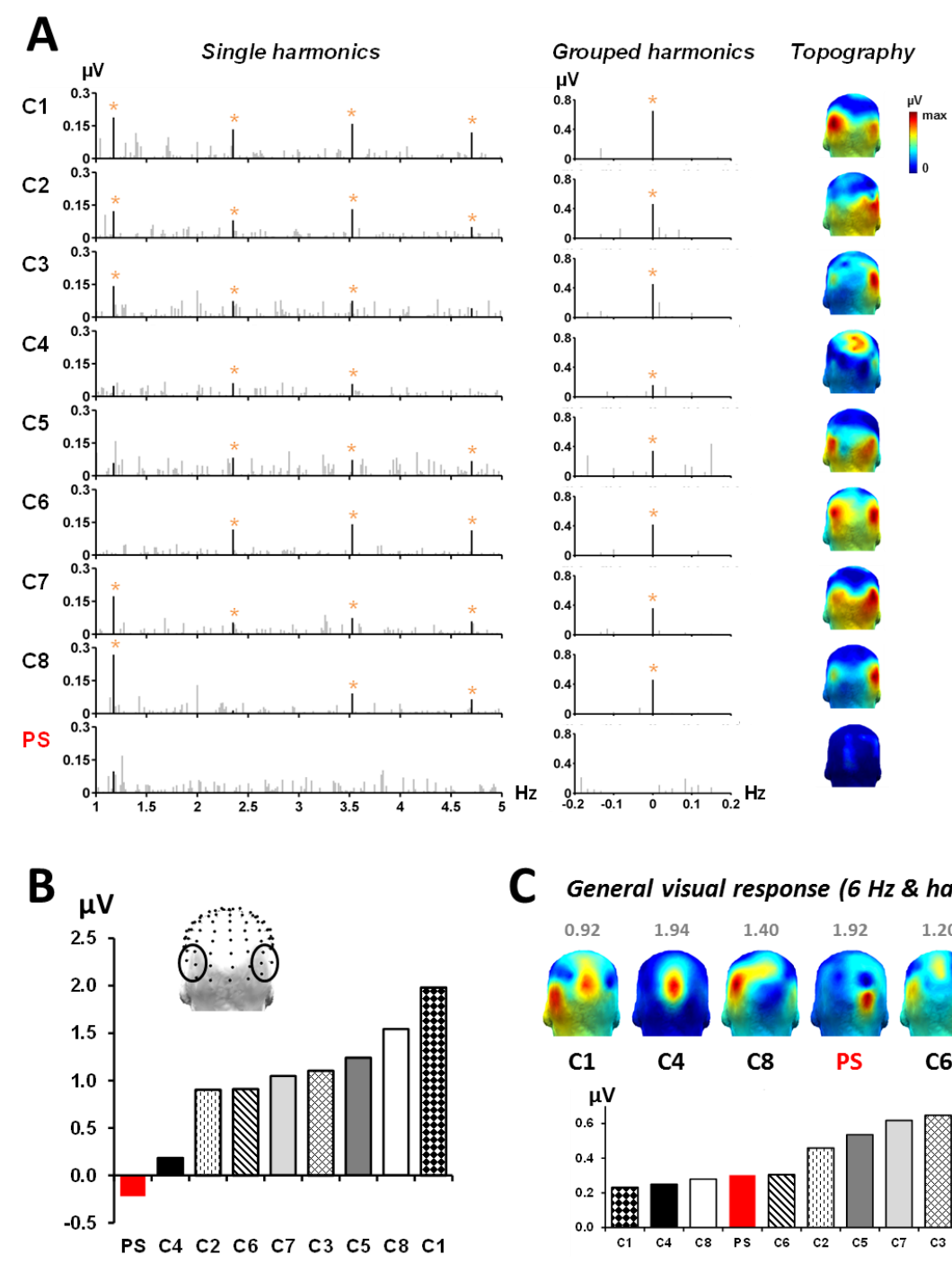

C General visual response (6 Hz \& harmonics)

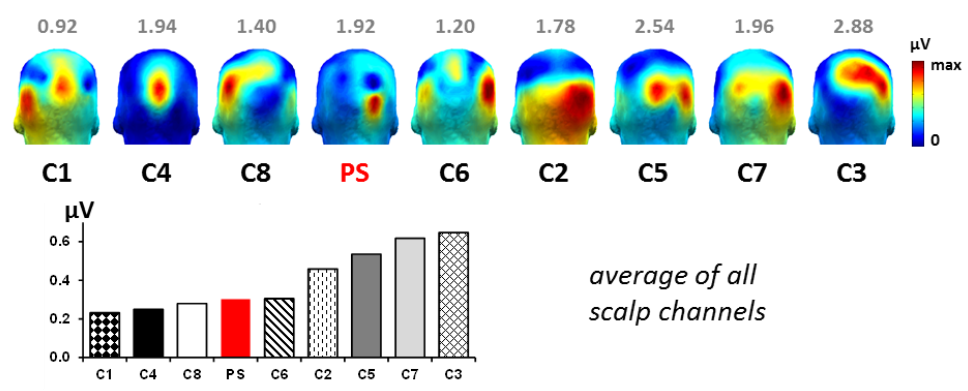

Figure 9. Responses of the prosopagnosic patient PS in the oddball FPVS FI experiment (from LiuShuang et al., 2016). 
A. Individual frequency-domain spectra and summed harmonics of the FI response at the bilateral occipitotemporal ROIs, and scalp topographies, for age- and gender-matched control participants (C1 - C8) and PS. For control participants, colors are scaled according to their individual maxima, whereas PS's topographical maps are scaled according to the lowest occipito-temporal response among control participants (C4). Responses were distributed around bilateral occipito-temporal channels in controls, with a right hemispheric dominance in the majority of participants (6/8), as in Liu-Shuang et al. (2014). B. Sorted face individuation responses in the bilateral occipito-temporal ROIs (channel composition shown on the blank 3D head). Contrary to controls, PS does not show any FI response above noise level. C. Sorted general visual responses at $6 \mathrm{~Hz}$ (average of all channels). PS had a significant general visual response at $5.88 \mathrm{~Hz}$, in the normal range. She also had a significant, normal range generic face categorization response (i.e., faces vs. objects; Liu-Shuang et al., 2016).

\subsubsection{Neural specificity and sources}

\section{Specificity of the neural FI response}

How specific is the individuation response to faces in this paradigm, i.e., could a similar response (in terms of summed-harmonic amplitude, scalp topography, harmonic frequency amplitude distribution, etc.) also be observed for exemplars of non-face objects? Note that finding a significant individuation response for non-face object categories would not at all undermine the validity of the approach to measure FI, as long as the neural individuation responses are quantitatively and qualitatively different for facial identity (if physical similarity between oddball and base exemplars is matched across designs). In this context, Lochy and colleagues (2018) tested participants undergoing weeklong training with novel multipart objects (Vuong et al., 2017) and found large individuation responses for exemplars at bilateral occipital sites, but without right hemispheric lateralization. Interestingly, one group of participants instructed to explicitly individuate the objects in a "face-like" stimulus orientation showed an increase in the EEG individuation responses after training. In contrast, such learning effects were absent in a second group trained with the same objects turned upside-down, into a "non-face-like" orientation. These observations indicate that, all other things being equal, perceived face-likeness plays a role in the magnitude of the EEG individuation response. This finding also illustrates how the adult visual 
recognition system exploits familiar spatial configurations when learning new object categories (Lochy et al., 2017; see also Vuong et al., 2017).

In another extension of the oddball FPVS paradigm, Hagen and Tanaka (2019) compared the ability of novice and expert bird watchers to categorize birds at a family-level (e.g., oddball crows $v s$. a base pigeon) or at a (finer) species-level (e.g., oddball house finches $v s$. a base purple finch). While robust occipito-temporal differentiation responses were found in both novices and experts, these responses tended to be slightly more broadly distributed and include medial occipital channels compared to the spatial distribution of FI responses in the same participants. Note that in terms of physical differences, the base and oddball bird images differed more than face images (especially in the family-level condition). Exemplar individuation of birds was not tested in that study and, to our knowledge, individuation within other familiar object categories has not been tested so far with this paradigm.

Finally, a recent study (using a higher $12 \mathrm{~Hz} F$ rate) found that the human FI response to upright human faces was twice as large as to monkey faces (Damon et al., 2020). Importantly, this effect cannot be merely attributed to larger physical differences between human faces than between macaque faces (although this is likely to be the case in general, since the face might not be highly diagnostic for identity in macaques; Rossion \& Taubert, 2019) given that the amplitude difference between conditions disappeared when faces were presented upside-down.

Taken together, these findings show that the $F / n$ individuation responses for faces are specific (qualitatively and quantitatively), relative to other types of individuation/categorization responses.

\section{Neural sources}

The right hemispheric occipito-temporal topography (as described in Section 4.2) strengthens the validity of the present approach, as it is theoretically consistent with the localization of the face-selective cortical regions in the human brain reported by numerous neuroimaging studies (i.e., in the ventral occipito-temporal cortex (VOTC) and superior temporal sulcus (STS); 
Sergent et al., 1992; Haxby et al., 2000; Duchaine \& Yovel, 2015; Grill-Spector et al., 2017; Gao et al., 2018). More importantly, the regions that cause a selective inability to specifically recognize facial identity when lesioned are also located in the VOTC, with a clear right hemispheric dominance (Meadows, 1974; Sergent \& Signoret, 1992; Bouvier \& Engel, 2006; Barton, 2008; Jonas et al., 2012; Rossion, 2014; Jonas et al., 2015).

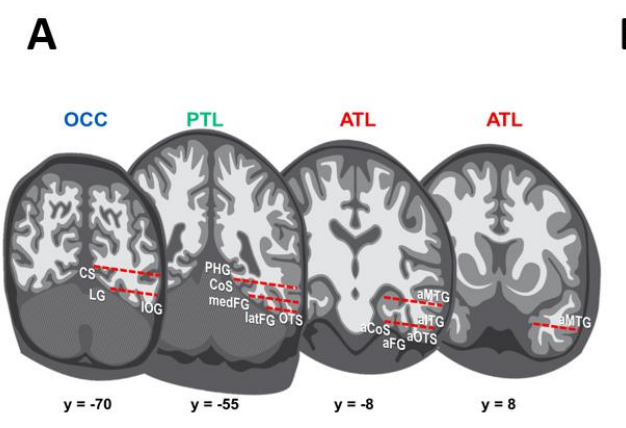

B
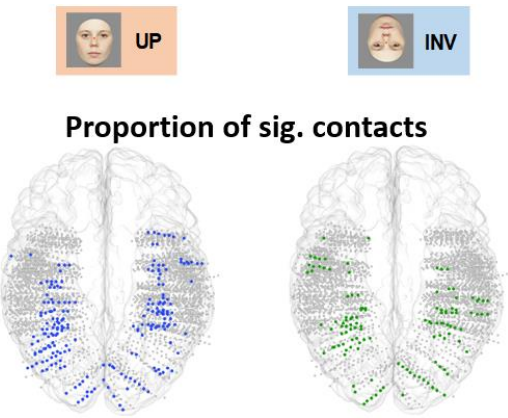

Amplitude of sig. contacts
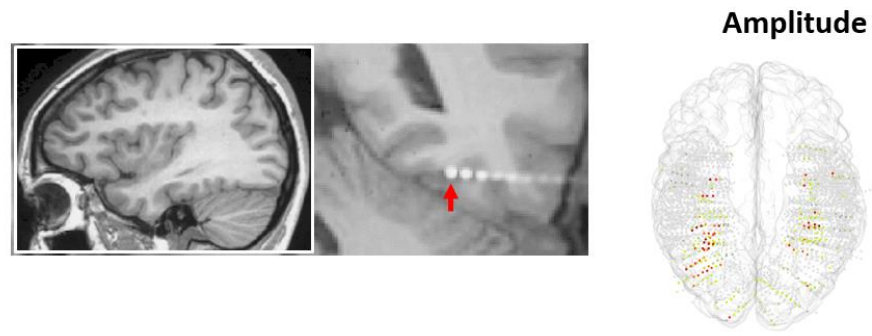

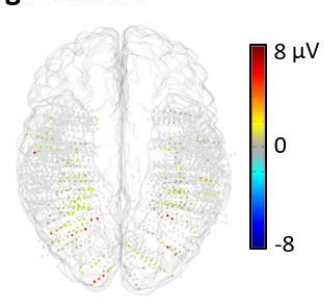

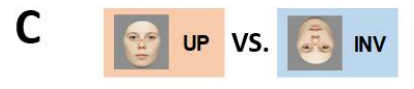

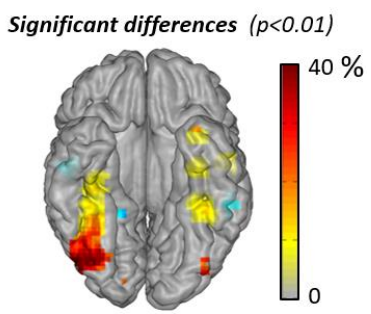

Significant differences (q FDR<0.05)

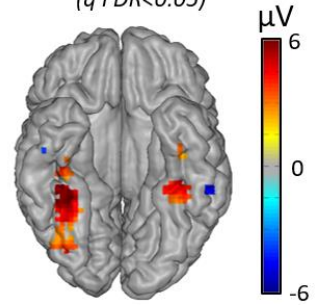

Figure 10. The neural basis of face individuation (from Jacques et al., submitted).

A. Intracerebral recordings in a large cohort $(\mathrm{N}=69)$ of temporal epilepsy patients presented with the oddball FPVS FI paradigm, at upright and inverted face orientations (Jacques et al., submitted; see Jonas et al., 2016; Rossion et al., 2018 for general methodological information). Across individual brains, a total of 3,825 electrode contacts were recorded in the grey matter of the VOTC. The bottom panel shows an electrode contact in the right lateral fusiform gyrus. Patients were tested with 2 to 4 stimulation sequences. B. Proportion of significant FI contacts relative to recoded contacts ( $<<0.001$, against noise level) for upright and inverted faces, and significant differences $(\mathrm{p}<0.01)$ in the proportion of significant FI contacts between upright and inverted faces. C. Amplitude of the FI response on the significant contacts, and significant difference maps $(\mathrm{p}<0.05)$ between upright and inverted faces. The FI response selective to upright faces is distributed, but confined to a strip of cortex outside of low-level retinotopic areas, from the lateral inferior occipital gyrus to the anterior fusiform gyrus (anterior to the so-called "Fusiform Face Area", "FFA"), with a strong right hemispheric dominance. In the individually, anatomically defined right lateral inferior occipital gyrus and fusiform gyrus, the amplitude decrease is of 53\% and 56\% respectively, in striking correspondence with scalp data in the neurotypical population (Figure 7). Note the near absence of response 
in the temporal pole, possibly due to the use of unfamiliar faces (i.e., facial identities devoid of semantic associations). $\mathrm{OCC}=$ Occipital region; $\mathrm{PTL}=$ Posterior Temporal Lobe; ATL $=$ Anterior Temporal Lobe .

The neural localization of the FI response was recently, more precisely explored by testing the paradigm in a large group $(\mathrm{N}=69)$ of temporal epileptic patients implanted with intracerebral EEG electrodes in the VOTC (Jacques et al., submitted; Figure 10). Interestingly, significant responses found in lower-level visual regions were eliminated when computing the difference between responses to upright $v s$. inverted faces. When isolating high-level (upright) FI responses this way, mapping the proportion of significant contacts and their amplitude both clearly identified a strip of cortex running from the lateral inferior occipital gyrus through the lateral fusiform gyrus, including its anterior section (Figure 10). However, despite extensive coverage in the most anterior sections of the ventral anterior temporal lobe (vATL), there were nearly no responses in this region, in particular in the temporal pole. The lack of FI responses in anterior sections of the vATL may be explained by the use of unfamiliar faces, which are not associated with semantic information. Although this exploration was limited to the VOTC, these observations are strikingly in line with the localization of brain lesions causing prosopagnosia (Meadows, 1974; Sergent \& Signoret, 1992; Bouvier \& Engel, 2006; Barton, 2008) and the effect of intracerebral stimulation (Jonas et al., 2012; Jonas et al., 2015) or even transcranial magnetic stimulation (TMS; Pitcher et al., 2007; Ambrus et al., 2016) on FI.

Note that these findings on the neural basis of FI with intracerebral recordings with an oddball FPVS paradigm are not redundant with findings from other approaches, such as fMRI, relying either on adaptation/repetition suppression (Grill- Spector et al., 2006) or more recently on Multivariate Pattern Analysis (MVPA; e.g., Nestor et al., 2011). MVPA-fMRI studies have been based on the rationale that there must be reliable differences in raw signal across a number of distributed locations (voxels) to different facial identities. If this is the case, patterns of activation - usually across a large chunk of cortex - for two presentations of the same face identity (e.g., face 
A) should be better correlated than for two different face identities (face A vs. face B) (i.e., the pattern for face A allows to "predict/decode" above chance level whether face A is presented again as compared to face B or face $\mathrm{C}$, etc.). Unfortunately, at this level of organization, with every voxel in conventional fMRI containing millions of neurons (Logothetis, 2008), there is no reason whatsoever to expect reliable differences in the raw signal to different face identities. Therefore, while MVPA studies are sometimes considered successful at "decoding facial identity", their decoding accuracies for individual faces in the human VOTC often reflect image-based discriminations of a few images (i.e., little or no generalization across image changes), and have been rather low and found in fairly inconsistent regions across studies, including low-level visual regions (Kriegeskorte et al., 2007; Nestor et al., 2011; Anzellotti et al., 2014; Goesaert and Op de Beeck, 2013; Verosky et al., 2013; Guntupalli et al., 2017; VanRullen \& Reddy, 2019; see also Davidesco et al., 2014 for ECoG studies; for critical views, see Rossion, 2014; Kanwisher, 2017). This is the reason why fMRI-adaptation to unfamiliar facial identities, which is based on the same principle as the paradigm reviewed here (see Section 6 below) constitutes a much more reliable approach to measure FI (Rossion, 2014) ${ }^{8}$.

Yet, fMRI-adaptation effects have been described essentially in pre-defined face-selective regions of the VOTC, such as the inferior occipital gyrus (IOG, or "Occipital Face Area”, OFA) and the mid-fusiform gyrus (FG or " "Fusiform Face Area", FFA) (e.g., Gauthier et al., 2000; Schiltz et al., 2006; Davies-Thompson et al., 2009; Ramon et al., 2010; Ewbank et al., 2013; Hermann et al., 2017; Hugues et al., 2019; see also Puce et al., 1999; Engell \& McCarthy, 2010 for such effects with human electrocorticography, ECoG) but with little evidence for clear right

\footnotetext{
${ }^{8}$ Note that MVPA is simply an analysis approach that could also be used with fMRI-adaptation data or data obtained in the present paradigm with scalp or intracerebral EEG. Usually, however,, this approach relies on the assumption that "information" is widely distributed in the brain, against the view of localized centers, or even of specialized neural networks.
} 
hemispheric lateralization. Moreover, fMRI only indirectly records neural activity, and the severe magnetic susceptibility artifacts arising from the ear canals seriously limits the ability of this method to explore VOTC regions located anteriorly to the mid-fusiform gyrus, i.e., the vATL (Wandell, 2011; Axelrod \& Yovel, 2013; Rossion et al., 2018). Hence, the more global exploration of the neural basis of FI afforded by the current oddball FPVS approach combined with human intracerebral recordings is especially valuable (Jacques et al. submitted).

A final line of evidence supporting the validity of our paradigm is the relationship between the oddball FI response and the effects of intracerebral electrical stimulation. Following such stimulation, a patient included in the study of Jacques et al. (submitted) experienced transient palinopsia limited to faces: she perceived facial features from a (variable from trial to trial) known familiar facial identity overlaid on the currently perceived face (a person or a picture in front of the patient; Jonas et al., 2018). The eloquent site of stimulation was located in the lateral section of the right middle fusiform gyrus, corresponding to the so-called FFA (Kanwisher et al., 1997). Strikingly, this stimulation site was also associated with the largest FI amplitude in our paradigm, compared to more than a hundred other electrode contact sites (Figure 11). Observations such as these, even based on a single case, again strongly support the functional relevance of the oddball FPVS approach to measure human FI. 
A

Localization of sEEG contacts (with functional face localizer results)

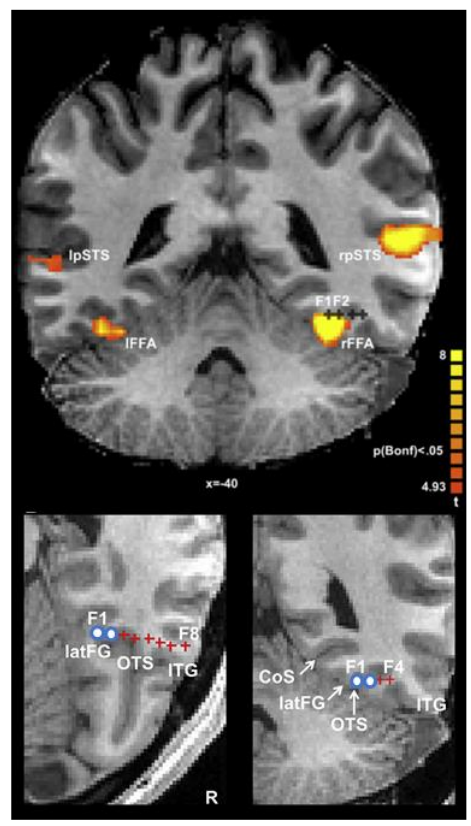

C

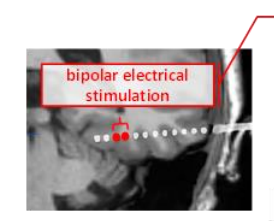

Famous face naming task

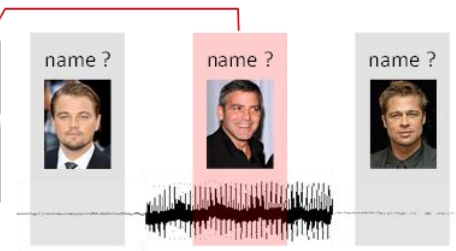

B

FPVS face individuation experiment
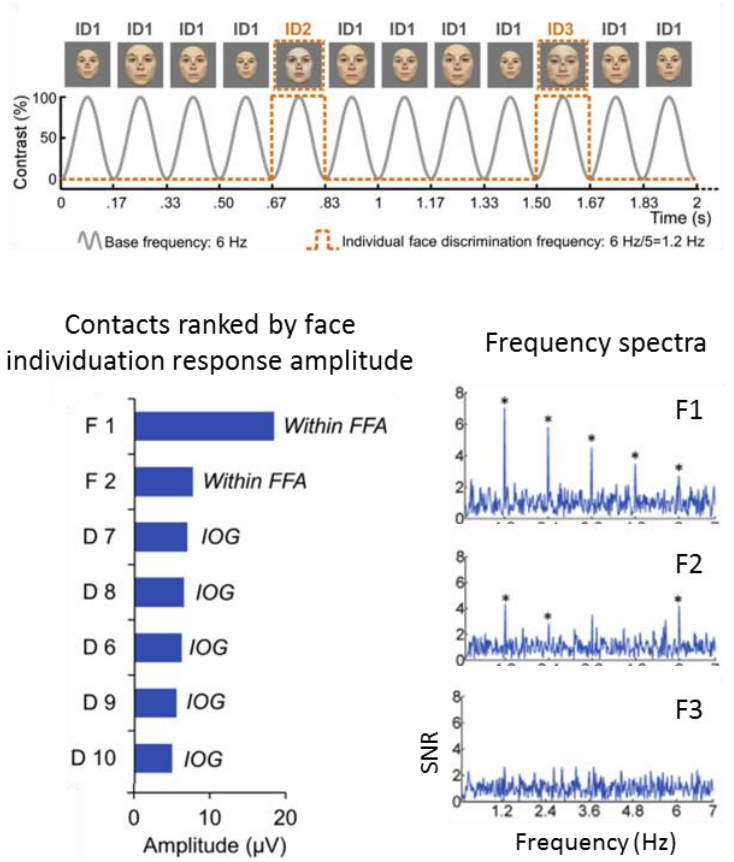

Frequency spectra

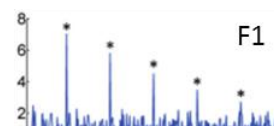

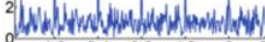
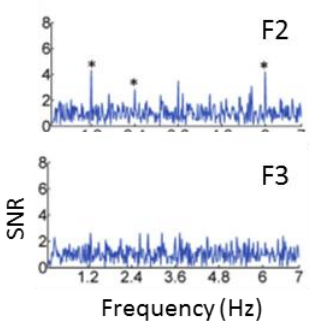

"I saw you with eyes and ears which were not yours"

"...the ears were not yours, they were those of somebody else"

"...they were not your eyes, they were the eyes of someone I had already seen, maybe coming from the images you showed me earlier"

Figure 11. Face individuation response localization predictive of transient palinopsia provoked by electrical stimulation during intracerebral recording (from Jonas et al., 2018).

A. Localization of intracerebral electrode contacts implanted in a patient with pharmaco-resistant epilepsy. The localization of the electrode $F$ is shown, relative to face-selective regions, defined with an fMRI functional localizer in this patient. The contacts F1-F2 fall within the right FFA. B. The patient completed 4 sequences of the FI paradigm reviewed here. Contact F1 showed the strongest FI response, followed by F2, as demonstrated by the clear peaks at the oddball face identity change frequency and its harmonics in the frequency-domain SNR spectra. C. Electrical stimulation of contacts F1-F2 only elicited vivid face hallucinations in the patient, who reported seeing familiar facial identities (that she was unable to identify) mixed with currently viewed faces (palinopsia) (see Jonas et al., 2018 for methodology). 


\subsubsection{Summary}

In summary, a wealth of evidence supports the validity of the FI measure in this oddball FPVS paradigm. Even though it is often based on the repetition of a single image of an unfamiliar facial identity (which varies across stimulation sequences), the response is far from being driven by low-level physical image properties. Hence, it is strongly reduced by contrast negation and inversion, two manipulations that preserve physical differences between the base and oddball stimuli but reduce the sensitivity to facial identity in neurotypical human adults. The FI response also generalizes across changes of size, luminance, and even head view, is absent in a welldocumented case of (acquired) prosopagnosia, and directly relates to cortical stimulation sites leading to impairments in face identity recognition, as well as changes in face perception (i.e., the subjective experience of the face). Scalp topographies and intracerebral recordings show that the FI response elicited with this paradigm does not occur anywhere, or over low-level visual regions, but is instead in agreement with the known right occipito-temporal dominance of the neural sources of human FI. Admittedly, the validity of a behavioral or neurofunctional measure in Systems Neuroscience cannot be established once and for all: it is based on a progressive accumulation of data, and the limits of this validity will only become clearer with further studies using the oddball FPVS framework.

\subsection{Objectivity, sensitivity, reliability}

\subsubsection{Objectivity in $\mathrm{Fl}$ response identification and quantification}

Objectivity in cognitive neuroscience research is important for reducing experimenter bias and for producing unambiguous results. According to a current popular view, objectivity is attained by reducing a priori experimenter selections, such as pre-defined spatial regions or time/frequency ranges of interest. This view favors data-driven methods, such as MVPA decoding approaches in EEG or MEG (e.g., Nestor et al., 2011; Carlson et al., 2013; Cichy et al., 2014; Vida et al., 2017), that often assess the brain's responses across all recording channels and all variations of space and 
time (and frequency), in order to find differences between stimulation conditions. The paradigm presented here is based on a radically opposite view of objectivity: that a priori assumptions may enable the unambiguous identification of processes of interest. Specifically, in this latter view, objectivity derives from the responses being specifically "tagged" at a priori, experimenter-defined frequencies (e.g., $1.2 \mathrm{~Hz}$ and $6 \mathrm{~Hz}$, and their harmonics). While there is experimenter choice in selecting the stimulation frequencies, there can be no bias in data analysis at this level: the stimulation frequencies define the exact frequencies-of-interest for analysis (unlike in standard ERP studies, in which identification of responses depends on a subjective definition of signal components against noise in the time-domain). Thus, in the absence of a response at those predefined frequencies, it is unnecessary - and incorrect - to explore other frequencies in the EEG spectrum in the hope of finding another response of interest.

Evaluating response significance at the frequencies-of-interest can be done in a straightforward manner by means of a quantitative/statistical computation relative to the neighboring frequency bins (see Box 1; see also Section 8 for methodological guidelines). Thanks to the high frequency resolution (afforded by the Fourier transform of a long EEG epoch, e.g., 60 $\mathrm{s}$, the response at frequencies-of-interest ("signal") are readily visible in the form of "peaks" (Figure 3) and can be compared to the amplitude of the surrounding frequencies that reflect baseline neural activity and potential recording artifacts (collectively termed "noise") ${ }^{9}$. Thus, the signal can be easily assessed against noise via baseline-subtraction, signal-to-noise ratio (SNR) calculation, or a statistic (typically a Z-score ${ }^{10}$ ) transformation (Srinivasan et al., 1999; Meigen \&

\footnotetext{
${ }^{9}$ A non-linear interaction of signal and noise at the frequencies-of-interest can lead to overestimation of the signal, although this may be negligible for high SNR signals (e.g., Bach \& Meigen, 1999).

${ }^{10}$ When neighboring bins are used, under the null hypothesis, the value at the frequency bin of interest is considered as being sampled from the same distribution of the noise. However, in practice, the EEG spectrum transformation can be performed by removing the frequency bin with the highest value to avoid projecting the signal into the noise (creating local symmetric decreases around the signal, e.g. figures in Liu-Shuang et al., 2014). The frequency bin with the smallest value would also be removed to balance out the effect of this procedure. Moreover, the two closest
} 
Bach, 1999; Rossion et al., 2012) ${ }^{11}$. These Z-scores values can also be used to determine the significance of FI response according to a statistical criterion (e.g., Z>3.1 $=p<0.001)^{12}$. Since the hypothesis is of signal>noise level, a one-tailed statistical test should be used (even if reviewers tell you otherwise!).

Since the initial report of the paradigm (i.e., Liu-Shuang et al., 2014), we have developed means to address multi-harmonic responses. While response quantification and significance assessment had originally been applied separately at each harmonic frequency-of-interest $(F, 2 F$, $3 F$, etc.), we now combine the amplitudes at these harmonics along with their respective noise, such that an overall aggregated FI response can be quantified and statistically evaluated (Retter \& Rossion, 2016). Hence, for the quantification of response amplitude (expressed in microvolts), the amplitude of each signal harmonic is summed along with its respective baseline noise and then isolated from noise by computing baseline-corrected amplitudes (typically a simple baselinesubtraction; Figure 3; Box 1). Note that while some subjectivity remains during data analysis (e.g., which EEG channels to consider, how to compare responses across conditions and individuals, etc.), these issues typically concern electrophysiological research in general, and are not specific to this paradigm (see Figure 21 for analysis steps).

frequency bin neighbors are often removed from the calculation to avoid any potential remaining effect of spectral "leakage" across frequency bins (see e.g., Yan et al., 2019).

${ }^{11}$ Typically, in the studies using the oddball FPVS paradigm to measure FI, 20 frequency bins - 10 on each side of the frequency bin of interest (a bandwidth of $\sim 0.3 \mathrm{~Hz}$ ) - are used to estimate the noise. Assuming that the EEG noise is randomly distributed in this small frequency window, finding the highest response at the frequency bin of interest is by chance would occur less than $5 \%$ of the time (i.e., $1 / 21=0.048$ or a $\mathrm{p}$ value $<0.05$ ). However, the number of frequency bins should be adapted to the spectral resolution (i.e., a smaller number of bins can be used if spectral resolution is lower, e.g., Leleu et al., 2019; Retter et al., 2020). Note that in contrast, in standard ERP paradigms, it is unclear how to objectively evaluate responses (e.g., in terms of SNR), due to a lack of objective signal vs. noise definition. It may also be noted that a non-linear interaction of signal and noise at the frequencies-of-interest can lead to overestimation of the signal, although this may be negligable for high SNR signals (e.g., Bach \& Meigen, 1999).

${ }^{12}$ If the noise is not normally distributed, for instance when using a small number of frequency bins or if the first harmonic is contaminated by particularly high amplitudes at low EEG frequencies, a non-parametric statistical test of signal vs. noise could also be implemented. 


\subsubsection{Sensitivity}

In standard ERP studies, while the absolute magnitude of the components in microvolts is typically higher than the responses elicited in the current oddball FPVS paradigm, the modulations of these ERP components by experimental manipulations are relatively small (e.g., on the N170 or N250r, as discussed above). Due to this lack of sensitivity, significant effects are often not present at the level of single participants and relatively long testing durations are required. In contrast, FPVS generally provide high SNR responses, since the signal of interest is concentrated at specific frequencies while the noise is distributed across numerous frequency bins (Regan, 1989; Norcia et al., 2015). SNR is typically further bolstered by a high event repetition count, afforded by the use of relatively high stimulus presentation rates (e.g., 360 faces per minute at $6 \mathrm{~Hz}$ and 72 identity changes per minute at $1.2 \mathrm{~Hz}$ ).

In the oddball FPVS paradigm, further sensitivity is provided by two factors. First, the $F / n$ response benefits from adaptation to the repeated base facial identity (see section 6 on Mechanisms). Second, the response at $F / n$ is defined differentially from base face responses (see Section 4.1), i.e., it is a direct measure of FI that does not require the comparison of different conditions a posteriori (e.g., one in which the same face is repeated and one in which different faces are presented, as in, e.g., Rossion \& Boremanse, 2011; Rossion et al., 2012). Indeed, in this paradigm, general visual responses related to pixel intensity, shape, or faces are captured at $F$, while only responses unique to detecting identity changes are captured at $F / n$. By setting the baseline activity to a rich face-related response, rather than to a uniform visual field, only the FI response projects to $F / n$ and the contrast is maximized. This compares favorably to an experimental situation in which a repeated face is indirectly contrasted to a novel face (i.e., novel face $v s$. uniform background, compared to repeated face vs. uniform background). In the latter case, most of the signal fluctuations are due to face stimulus onset, decreasing sensitivity for specifically measuring FI. 
We used the current large-scale group analysis to quantify the sensitivity of this paradigm to measure FI ( $=130$; Supplementary Material for details). The SNR was high overall: at the level of individual oddball $F / n$ frequency harmonics $\left(2^{\text {nd }}, 3^{\text {rd }}\right.$, or $\left.4^{\text {th }}\right)$, the SNR could reach 2.5 to 3 , corresponding to a $150 \%$ to $200 \%$ amplitude increase relative to noise (Figure $\mathbf{4 A}$ ). Combining the harmonics, the group-level FI SNR went above 2, corresponding to a $100 \%$ signal increase (Liu-Shuang et al., 2014; Figure 12). Moreover, the individual level FI SNR, although quite variable (range: $0.91-3.22$ ), exceeded 1 in all but one subject in our sample. Here, only four 60 -s stimulation sequences were sufficient to obtain significant responses in $93 \%$ of neurotypical individuals, at a one-tailed threshold of $\mathrm{p}<0.05$ (Figure 12). Even when only considering a single minute of stimulation, significant and high-SNR FI responses were recorded in most individuals (although SNR is of course substantially augmented by increasing the number of stimulation sequences).

Overall, the sensitivity of the FPVS-oddball approach allows for a meaningful FI response to be obtained: 1) in a short amount of testing time; 2) with minimal and straightforward data processing (sometimes without artifact rejection/correction, e.g., Xu et al., 2017); and 3) with significance often attained at the individual subject level, such that results are highly reproducible across studies (see the next section). 
A

Histogram of face individuation response amplitudes

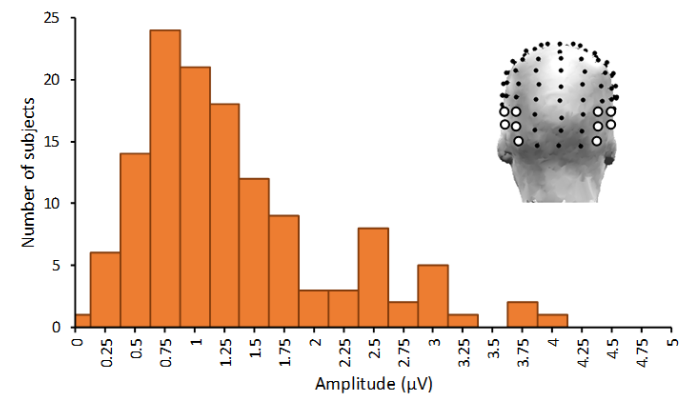

B Response significance

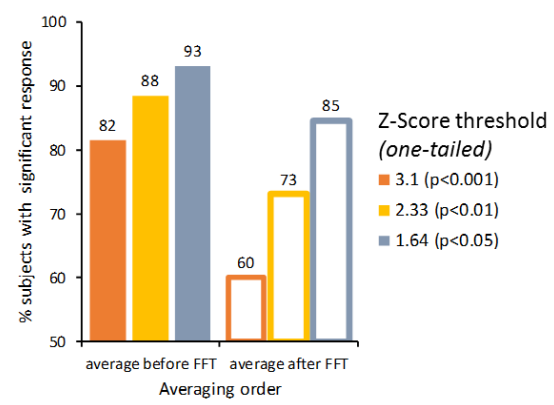

C

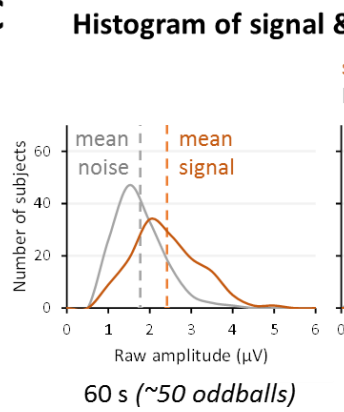

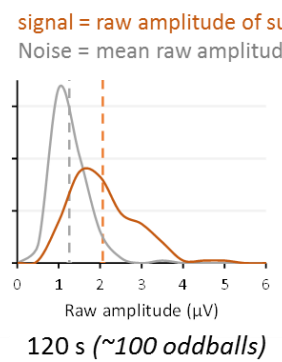
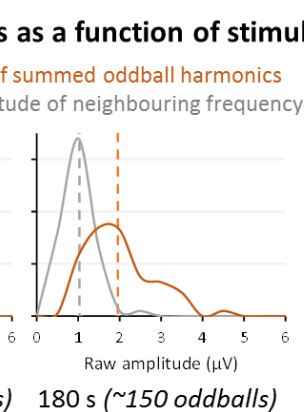

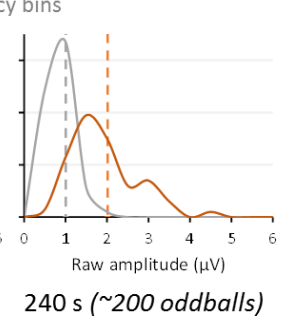

SNR as a function of stimulation duration

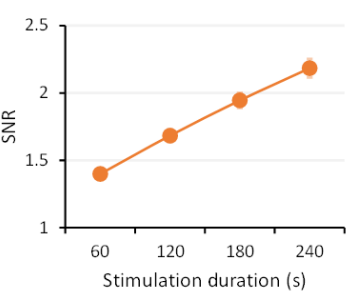

Figure 12. Face individuation response size and significance (large-scale group analysis).

A. Histogram of the FI response amplitudes across 130 participants, measured over a bilateral occipitotemporal ROI (channels shown on the head model). On average, the amplitude of FI responses is centered over $0.75-1 \mu \mathrm{V}$. Note that amplitudes are not normally distributed, but rather skewed towards lower amplitudes with a long rightward tail. B. Proportion of participants with significant FI responses at three levels of statistical significance, and for two analysis procedures: either averaging the EEG signal across the 4 sequence repetitions within subject data in the time-domain before the frequency-domain transform, or inversely, transforming data into the frequency-domain and averaging afterwards. Averaging in the timedomain reduces the contribution of responses that are not in phase across stimulation sequences (predominantly noise). Significant responses in a range of 80-90\% of individual participants can be reached when averaging before FFT even with only 4 stimulation sequences of 1 minute. Averaging after FFT reduces response amplitude and as a consequence, response significance. Note that robust differences between groups or conditions could still be found with this paradigm even if significant responses were not observed at the individual level (i.e., if a majority of participants had a non-significant FI response). C. Effect of the stimulation duration (number of sequence repetitions) on the FI response (here summed over oddball harmonics up to $6 \mathrm{~Hz}$ ), when time-domain averaging is done before FFT (the typical procedure). The distribution of raw amplitudes shows that increasing the stimulation duration decreases EEG noise in the surrounding frequency bins. As a result, signal-to-noise ratio (signal/noise) increases with the number of sequences, with an average increase of $\sim 50 \%$ from $60 \mathrm{~s}$ ( $\mathrm{SNR}=1.4$ ) to $240 \mathrm{~s}(\mathrm{SNR}=2.2)$ of stimulation. Note also that the SNR of single harmonics can be higher than the overall SNR (see Figure 4A). 


\subsubsection{Reliability}

An important issue is whether the oddball FPVS measure of FI is reliable at the group level and, most importantly, at the individual level. The test-retest reliability of this paradigm has been examined in three recent studies. Using a low-density channel system, Xu et al. (2017) showed that the variations of FI amplitudes of 49 individuals tested within the same recording session were highly reproducible (Cronbach's alpha $=0.87$ ). More recently, Dzhelyova et al. (2019) went further by testing 30 individuals and comparing their high-density EEG responses to four one minute sequences between recording sessions, two-months apart. They found a high correlation between participants' FI response amplitudes across sessions ( $r=0.79$; intraclass correlation coefficient $\mathrm{ICC}=0.87,95 \% \mathrm{CI}=[0.730 .94])$ (Figure 13). Impressively, the between-session reliability was almost identical to the within-session reliability.

Dzhelyova et al. (2019) also modeled the relationship between the number of stimulation sequences and reliability. Results suggested that high reliability could be obtained for even a single one-minute sequence, and that including five or more sequences would lead to near-perfect reliability of FI amplitude measures (Figure 13C). Additionally, classification analyses indicated that, despite some variability in the FI amplitude distribution across harmonic frequencies in individual observers, the overall profile was relatively stable within a given individual subject and provides an additional measure of single-subject reliability (Dzhelyova et al., 2019). Finally, this study also demonstrated that the scalp topography of the FI response was highly stable across time. On group-level data, the correlation coefficient across scalp electrodes (i.e., 128 data points per session) reached 0.96 . For individual data, response topography was most reliable across sessions over (right) occipito-temporal regions (range across right ROI electrodes: $r=0.64-0.79$, Figure 13), where the maximal face individuation response was observed (Figure 13). This is important because it validates the focus on these occipito-temporal regions of interest for comparisons across conditions: even if they only account for a (substantial) portion of the response (Figure 5), other electrodes on the scalp do not record highly reliable responses. 
The reliability of the (right) occipito-temporal topography of the FI response was also tested by Stacchi et al. (2019), after an even longer interval, 6 months, between recording sessions. These authors additionally manipulated the fixation position across various locations of the face (e.g., left eye, right eye, nose...) to take into account idiosyncrasies in preferred fixation position during face identity processing (e.g., Peterson \& Eckstein, 2013). Overall, despite acquiring only two stimulation sequences by condition, their data confirmed individual differences in preferred viewing positions, and notably also the reliability of the FI response pattern across viewing positions within each observer.

Taken together, these findings indicate that the oddball FPVS FI response is stable at the group level and also at the level of individual observers, although it can be recommended to concentrate the measurement on ROIs/channels carrying the largest amplitude, and to include at least 4 sequences of about 1 minute of testing (Figure 13). Unlike behavioral tests such as the CFMT (Wilmer et al., 2010), responses do not improve/increase at retest because subjects become familiar with the face stimuli or the task. In fact, while the same set of faces are used in the test and retest sessions in the FPVS studies above, it is worth mentioning again that the base face (i.e., the repeated identity) is randomly selected for each trial, as are the oddball facial identities. Hence, stimulation sequences differ between test and retest sessions for each individual participant, making the high reliability coefficients all the more remarkable. 
A
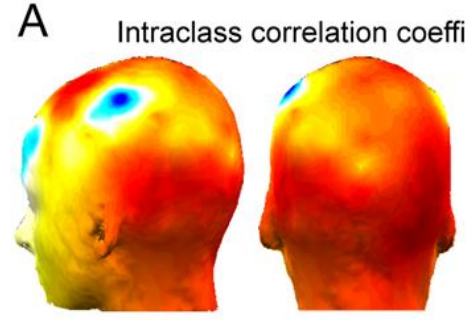

ICC $p$-values
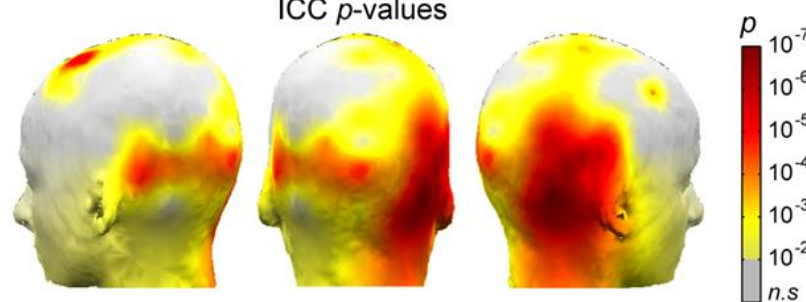

C

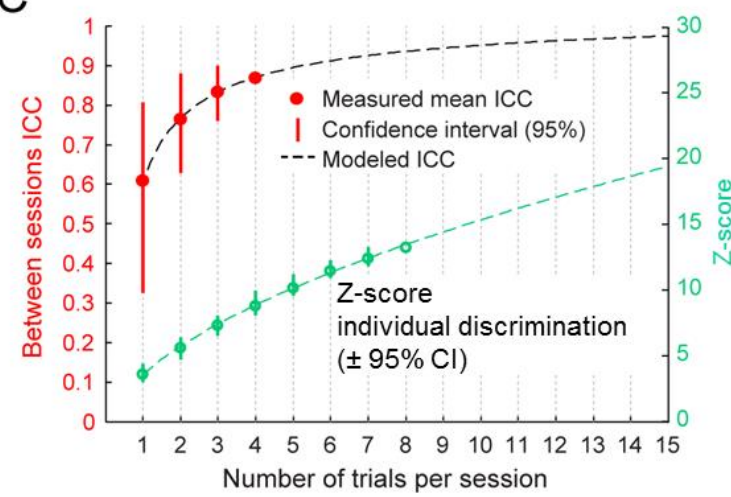

B

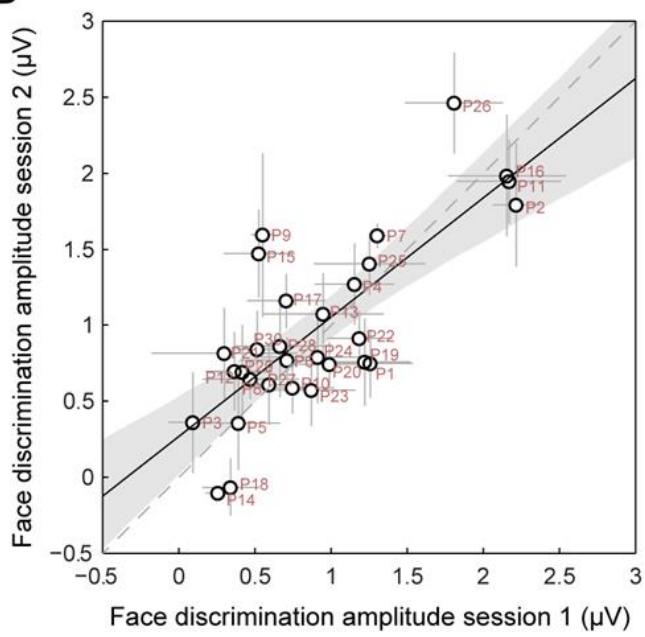

$\mathrm{D}$

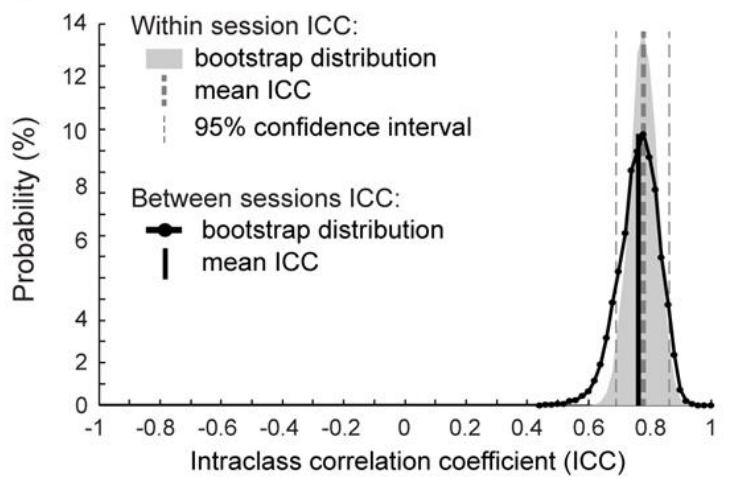

Figure 13. Test-retest reliability of the face individuation response (from Dzhelyova et al., 2019).

A. Pearson correlation coefficients across the two sessions ( 2 months apart) for each of the 128 channels, displayed as topographical maps (upper row) and the corresponding p-values (bottom row). Significant correlations ( $\mathrm{p}<0.01)$ are color-coded; non-significant correlations are shown in grey. B. Scatter plot showing the FI response $(1.2 \mathrm{~Hz})$ amplitude across the two sessions for all participants. Each data point represents the baseline-corrected amplitude summed across harmonic frequencies (up to $8.40 \mathrm{~Hz}$, excluding the $6 \mathrm{~Hz}$ general response) for the occipito-temporal ROI (LOT or ROT) per participant and averaged across the 4 trials of each session. Error bars indicate SEM across the 4 trials of the same session. C. Model estimation of the relationship between the number of stimulation sequences and the between-session reliability. Red dots indicate measured intraclass correlations for 1, 2, 3 or 4 (all) trials as well as the $95 \%$ confidence interval. The dotted line shows the fitted power-law function. D. Comparing within- and between-session FI response reliability. Bootstrap distribution of correlations for within-session (light grey filled) and between-sessions (black line) reliability estimates. Correlations were computed using 2 randomly selected trials either from the same session (within) or different sessions (between) for each bootstrap. Thick vertical lines correspond to the distribution mean and thin dotted lines indicate the $95 \%$ confidence interval for within-session correlations. 


\subsubsection{Summary}

Given its advantages in terms of sensitivity (and thus short experimental testing time), reliability, and objectivity, the oddball FPVS FI paradigm reviewed here is highly extendable to different populations. Its objectivity may be particularly appropriate for relating EEG responses across developmental groups, including infants and young children (for whom ERP components substantially differ) in order to clarify the developmental time course of FI. The paradigm is also well suited to study FI in clinical populations, including patients with prosopagnosia and individuals with autism spectrum disorder.

\section{Mechanisms}

\subsection{General neural mechanisms of frequency-tagged EEG responses}

What are the neural mechanisms driving the frequency-tagged responses observed in the EEG frequency-domain spectrum, both at the base rate $F$ (Figure 3 \& 14) and - most importantly - at the $F / n$ facial identity change rate? Generally, EEG is thought to reflect current flows at the postsynaptic level in extensive populations of neurons (Regan, 1989; Nunez \& Srinivasan 2005; Kirschstein \& Köhling, 2009). The basic neural mechanisms of EEG apply to FPVS responses, i.e., that a robust electrophysiological scalp response at the stimulus presentation frequency $F$ (Figure 14) originates from sufficiently large neural populations (Figure 10) responding at the rate of visual stimulation, to generate postsynaptic potentials in those regions of the cortex. Importantly, responses to each stimulation do not have to be perfectly synchronized in time (i.e., start/end at the exact same time and with the same shape) to generate a response exactly at the $F$ (or $F / n$ ) driving frequency in the EEG spectrum. That is, a series of individual responses occurring every $170 \mathrm{~ms}$ on average, even with a small time jitter in between response onsets, will lead to a narrow peak in the frequency spectrum at $5.88 \mathrm{~Hz}$ insofar as the EEG response is well accounted for by a $5.88 \mathrm{~Hz}$ sinewave in the Fourier decomposition (Figure 3). 

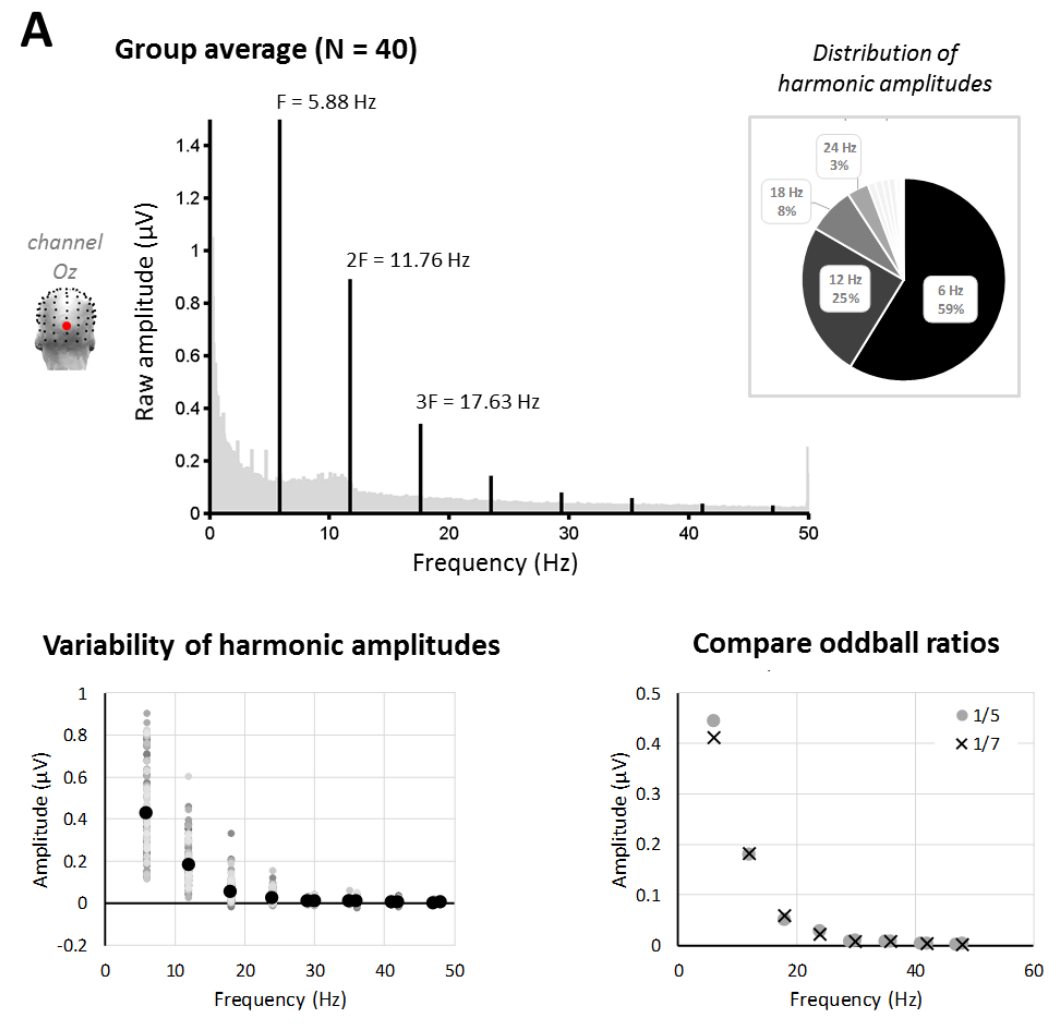

B

Proportional distribution of general visual response amplitude

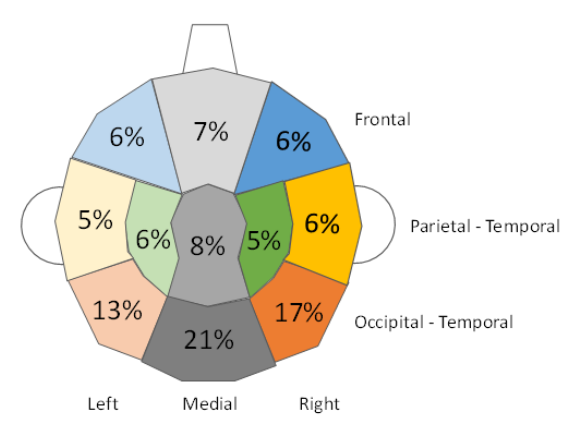

D Effect of inversion on harmonics

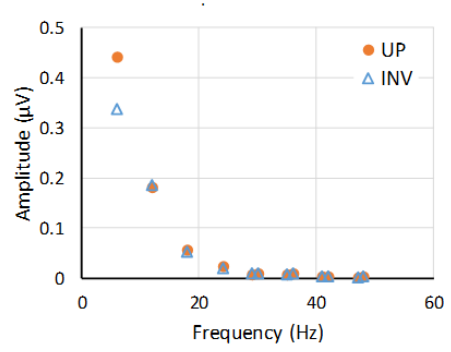

C Distribution of response amplitudes

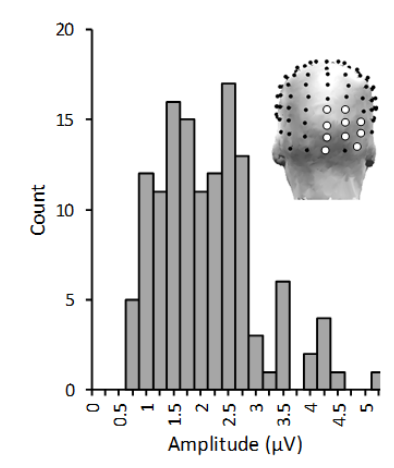

Effect of inversion on general visual response

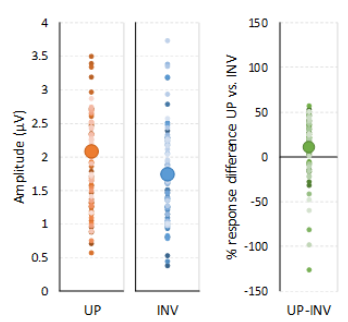

Figure 14. Responses at the face stimulus presentation rate $(F=5.88 \mathrm{~Hz})$, reflecting general visual processing (large-scale group analysis).

A. Properties of the harmonic distribution of the response to stimulus presentation at $F=5.88 \mathrm{~Hz}$. The general visual response decreases exponentially with increasing harmonics, with responses at the 
fundamental $5.88 \mathrm{~Hz}$ frequency accounting for nearly $60 \%$ of the total response, with the majority of the response contained below $30 \mathrm{~Hz}$. Again, there is no difference in the harmonic frequency amplitude distribution between oddball ratios (1/5 or 1/7). B. Distribution of the stimulus presentation rate response (amplitude) over the different ROIs (see Figure 4 for scalp topographies). C. Variability of response amplitudes across individuals. Response amplitudes within the medial occipital ROI are much more uniformly distributed across the 130 individuals than for the FI response (see Figure 4). D. Face inversion leads to a small reduction of this general visual response (10\% on average), driven almost exclusively by the first harmonic at $5.88 \mathrm{~Hz}$. Note that here the modulation by inversion is distinct from the classic face inversion effect shown in Figure 7, since it relates to a general response to face stimuli, not to the individuation of faces.

Researchers have debated whether the neural mechanisms of frequency-tagged responses differ from those of standard ERP responses. More precisely, it is debated whether frequencytagged responses reflect the mere linear summation of successive ERP-like responses, or rather the modulation of ongoing (i.e., pre-stimulus) neural oscillations (e.g., Capilla et al., 2011; Gruss et al., 2012; Keitel et al., 2014; Spaak et al., 2014; Notbohm et al., 2016; Zoefel et al., 2018). When framed as mutually exclusive hypotheses (i.e., "linear superimposition of ERPs" or "oscillation entrainment"), the question about the neural basis of frequency-tagged responses appears to be illposed, for several reasons. First, an EEG recording of a living organism is never flat (i.e., "zero activity"), so that time-locked responses to transient events always superimpose to ongoing neural activity, which can always - at least partly - be described in terms of oscillations (Makeig et al., 2002; see Rousselet et al., 2007).

Second, different stimulation conditions may be more in line with one or the other account, or a mixture of both. For instance, the linear superimposition account of EEG frequency-tagged responses is based on models of basic stimuli and simple stimulations (e.g., checkerboard reversal in Capilla et al., 2011, or responses to simple tones in the auditory domain; Galambos et al., 1981) which may not generalize well to more complex stimulation conditions such as used here. Third, it is well known that frequency-tagged EEG responses are larger in amplitude for certain frequency ranges, e.g., around $10 \mathrm{~Hz}$ for the first harmonic of low-level visual stimulation recorded at medial 
occipital sites (since Regan, 1966). However, contrary to a widespread assumption, this does not necessarily support the account of an entrainment of ongoing EEG (alpha) oscillations by the stimulation. Instead, if a low-level visual response (e.g., to a uniform luminance change, a grating orientation change or a checkerboard reversal) generates a $\sim 100 \mathrm{~ms}$ duration response, the stimulation frequency that will generate the largest first-harmonic EEG response will be of about $10 \mathrm{~Hz}$, fitting about 10 such responses by second. Below this frequency, fewer events are recorded per second, leading to a weaker SNR. Above this frequency, the individual responses overlap, interfering with each other, and potentially leading to a greatly reduced, or even absent, neural response. It follows that certain stimulus presentation frequencies are more or less appropriate for measuring different neural processes, based essentially on the duration (or time-constant) of the neural process elicited (Keysers \& Perret, 2002; Retter \& Rossion, 2016; Retter et al., 2020; see also Alonso-Prieto et al., 2013; Bekhtereva \& Müller, 2015; Collins et al., 2018).

Based on these considerations, instead of either of the two accounts mentioned above, the most parsimonious and plausible explanation of frequency-tagged responses appears to be in terms of nonlinear interactions between transient neural events (i.e., ERPs) that may partially overlap in time (Retter \& Rossion, 2016). Empirically, increasing overlap of successive neural responses has been shown to increase the oscillatory appearance of these neural responses (see Box 1). Importantly, the oscillatory shape of these responses does not necessarily reflect a simple linear response: instead, it could reflect a highly non-linear interaction between complex, ERP-like neural response. For example, while presenting sequences containing different facial identities at $2 \mathrm{~Hz}$ elicits very similar ERP-like response waveforms, the same sequences shown at $6 \mathrm{~Hz}$ lead to a more sinusoidal waveform over occipito-temporal sites (Alonso-Prieto et al., 2013). Note that this will have implications for the frequency-tuning profiles of frequency-tagged responses in the present context: see Section 7 below. 


\subsection{Face individuation mechanisms}

As mentioned in the description of the oddball FPVS paradigm in Section 4.1, the EEG response to oddball identity changes at $F / n$ is a differential response, reflecting electrophysiological activity specific to oddball vs. base facial identities in terms of amplitude and/or latency. However, as noted above, given that the spatial resolution of recorded scalp (or even intracerebral) EEG responses, i.e., at the scale of large populations of neurons, is thought to be much greater than the scale of distinct neural facial identity representations, there is no reason to expect that a given unfamiliar (e.g., oddball) facial identity would generate a different EEG response than another unfamiliar (e.g., base) facial identity. Hence, the most plausible explanation of the FI response observed in the current paradigm is that there is habituation or adaptation of the neural population response to the repeated base facial identity (A), such that the introduction of different facial identities at fixed intervals (B, C, D ...; Figure 1) leads to a periodic rebound (i.e., release from adaptation) of neural responses. Even though, as mentioned above, an oddball face could generate a decrease, increase or phase shift of neural activity, we look to repetition suppression (RS) as the most likely explanation of FI responses in this paradigm for two reasons.

First, RS effects have been well studied and documented with face stimuli in neuroimaging studies, in particular for the repetition of facial identity (Gauthier et al., 2000; Schiltz et al., 2006; Davies-Thompson et al., 2009; Ramon et al., 2010; Ewbank et al., 2013; Hermann et al., 2017; Hugues et al., 2019; see also Puce et al., 1999; Engell \& McCarthy, 2014 for such effects with human electrocorticography, ECoG). As discussed above, RS effects have also been reported at the level of ERP responses to faces, either at the category level (e.g., a decrease of the N170 amplitude to a face following another face compared to a face following an object, Kovacs et al., 2006; Eimer et al., 2010), or the facial identity level (e.g., an amplitude decrease to a repeated face identity, Barrett et al., 1988; Jacques et al., 2007; Vizioli et al., 2010; see Rossion \& Jacques, 2011; Schweinberger \& Neumann, 2016). Moreover, RS effects may also account for the reduction of 
EEG response amplitudes to FPVS sequences of repeated facial identities $v s$. different facial identities (e.g., Rossion \& Boremanse, 2011; Alonso-Prieto et al., 2013; Nemrodov et al., 2015).

Second, the present paradigm utilizes stimulus repetitions and changes at short time scales (hundreds of ms), which are appropriate to produce RS effects (which may be produced across many timescales, potentially relating to different neural mechanisms; Grill-Spector et al., 2006; Henson, 2016). By presenting face stimuli at relatively fast rates, we may produce interference of overlapping neural responses specific to a repeated facial identity (see Section 4.1, particularly Box 1). Interestingly, in the study of Alonso-Prieto et al. (2013), there was no difference in the FPVS EEG response to repeated $v s$. different facial identities at low face stimulus presentation rates (e.g., 1 or $2 \mathrm{~Hz}$ ). In contrast, differences between FPVS EEG responses to repeated $v s$. different facial identities were found at a higher range (at about $4-8 \mathrm{H}$; and also at $3.5 \mathrm{~Hz}$ in an earlier study, Rossion \& Boremanse, 2011). This suggests that potential RS mechanisms in FPVS EEG designs with high face stimulus presentation rates, including the oddball paradigm here, typically with $F=$ $6 \mathrm{~Hz}$, are affected by short identity repetition time intervals, beyond absolute image viewing time.

Further evidence to this effect was provided by Nemrodov et al. (2015), who reported that decreased FPVS EEG responses to a repeated facial identity at $6 \mathrm{~Hz}$ occurred within only hundreds of milliseconds, and afterwards remained stable, over the right occipito-temporal cortex. Finally, with the current oddball FPVS paradigm, the largest FI EEG responses, consistently occurring at $F / n=1 \mathrm{~Hz}$, are found between $F=4-6 \mathrm{~Hz}$ (Figure 20; Retter et al., in preparation). In that case, decreased FI responses at the lowest rate, $F=3 \mathrm{~Hz}$, could potentially be explained by decreased base facial identity RS effects, given that there were only two base repetitions, of $333 \mathrm{~ms}$ each, between each identity oddball (Figure 20; compare to Retter et al., 2020; see also Section 8.1.2).

It should be noted that criticisms of repetition/oddball paradigms, and the inferences drawn from their results, have been made at several levels. First, essentially for fMRI studies, RS effects have been criticized for over-interpreting their findings in terms of revealing the "nature of representations", or what is coded at the level of subpopulations of neurons. This overinterpretation 
results from a reverse inference error. That is, if two different populations of neurons are intermingled within the brain same region, RS effects can potentially reveal this difference (GrillSpector \& Malach, 2001). However, the reverse is not true: the presence of a RS effect does not necessarily imply that there are two distinct populations of neurons, since the responses from a single neural population could also differ for both stimuli, e.g., with different timing parameters. Therefore, one should be cautious when making inferences from large-scale RS effects to single neuronal responses (Sawamura \& Vogel 2006). Provided that this kind of overinterpretation is avoided, RS effects at the larger scale can be valid measures of the brain's sensitivity to a property of interest.

Second, RS effects have sometimes been defined as non-specific, since they supposedly occur in many brain regions beyond face-selective cortical areas (Mur et al., 2010). However, this limitation is not intrinsic to paradigms based on the principle of RS, but rather applicable to poorly controlled designs and stimuli with prominent image-based effects. As discussed above, RS effects to repeated identities appear to largely reflect high-level, facial identity selective responses (see Section 5.1.1). With the present oddball FPVS paradigm, the maximal FI response is located in the lateral inferior occipital and fusiform gyri with intracerebral EEG (Section 5.1.4), and responses on the scalp are maximal over the right occipito-temporal cortex (Figure 5). In agreement, the potential RS effects reported in previous FPVS EEG studies for facial identity were also located maximally over the (right) occipito-temporal cortex (Rossion \& Boremanse, 2011; Alonso-Prieto et al., 2013; Nemrodov et al., 2015). Still, it is possible that these high-level effects are influenced by low-level properties of the face images (see Nemrodov et al., 2015), as discussed earlier in the context of the present paradigm (see Section 5.1).

Finally, it is worth noting that over the past years, RS effects (and other stimulus repetitionrelated effects) have been tentatively explained under the framework of predictive coding (e.g., Summerfield et al., 2008; Todorovic \& de Lange, 2012; Grotheer \& Kovács, 2015), according to which the sensory inputs, together with our prior experiences, are used to form predictions of 
upcoming events to ensure efficient processing (Rao and Ballard, 1999; Friston, 2005). In this context, some authors have referred to change detection or oddball responses, as "prediction errors" rather than as reflecting adaptation (e.g., Lieder et al., 2013; Stefanics et al., 2018). However, even in a highly predictive context (only two unfamiliar face identities as frequent and deviant, with no size change), expectation and surprise do not seem to contribute to the FI response (Feuerriegel et al., 2018).

More generally, in our view, this new terminology - and the computational description that goes with it - offers no increase in explanatory power, especially in the present context, and may in fact cause more confusion than anything else (see also O'Shea, 2015; Walsh et al., 2020). It has long been known that the visual system (and the nervous system in general) does not respond to absolute properties of stimuli (e.g., light intensity) but to spatial and temporal differences of inputs (i.e., contrast), from the retina to at all levels of organization and processing. Changes of input can be detected at many levels of the neural circuitry, depending not only on the changes in physical input properties but also on the long-term and recent experience of these neural circuits.

In the case of the FI response reviewed here, the large effect of stimulus inversion alone makes it quite clear that this response is not purely dependent on physical characteristics of the stimuli but depends on the system's knowledge of facial structure (derived from past experience), in line with a constructivist view of (visual) recognition (Helmholtz, 1867; Gregory, 1980; see also Purves et al., 2015; Rossion \& Retter, 2020). Based on these considerations, instead of a generic functional/computational description in terms of "predictive coding" or "prediction error", what is needed here is a specific mechanistic account in terms of neurophysiological activity of populations of neurons in a large-scale occipito-temporal cortical circuit (i.e., a cortical reflex circuit; Sherrington, 1947), automatically activated to individuate human faces.

While RS phenomena have been described with large-scale recordings, such as described above with human EEG and hemodynamic changes in fMRI, they have also been reported across multiple spatial scales in the nervous system, down to individual cortical neurons in monkeys 
(Grill-Spector et al., 2006; Henson, 2016). Generally, across various levels of brain organization, RS effects reveal neural sensitivity to certain differences between stimuli that cannot be disclosed by simply comparing the responses to the stimuli in isolation. While the precise neural mechanisms of RS effects remain unknown, it is likely that they are best understood at the level of small neuronal populations, a level at which various interesting hypotheses have been proposed (e.g., neuronal fatigue, sharpening, or facilitation; see Grill-Spector et al., 2006). We thus posit that clarifying the exact neural mechanisms of the present FI paradigm will require recordings down to the columnar and single-neuron levels of brain organization, in many regions of the cortical circuit. Given that monkeys are naturally poor at this FI function (Rossion \& Taubert, 2019), such recordings will have to be performed in the human brain (e.g., Rey et al., 2020), particularly in (right) ventral occipito-temporal structures where the largest FI effects are disclosed; Figure 10).

\section{Insights into face individuation}

In the above sections, we have demonstrated the advantages of the oddball FPVS paradigm in terms of its validity, objectivity, sensitivity, and reliability for measuring high-level FI. Now, we will briefly summarize and discuss how this paradigm has contributed to furthering our understanding of human facial identity processing, taking examples from published and unpublished data, as well as the current large-scale analysis.

\subsection{Interindividual variability and relationship with behavior}

The FI response varies substantially across individuals in terms of scalp EEG amplitude (e.g., Liu-Shuang et al., 2014; Xu et al., 2017; Stacchi et al., 2019a; Stacchi et al., 2019b; Dzhelyova et al., 2019; Figures 7D \& 12A), ranging between $\sim 0.25 \mu \mathrm{V}-4 \mu \mathrm{V}$ in our large-scale group analysis $(\mathrm{N}=130$; again, see Supplementary Materials for methods). Thus, individuals can have up to over ten-fold differences in their FI response amplitude. This large variability is likely due to multiple factors. Some factors may relate directly to the FI function, such as the size and spatial extent of the neural population (specifically) involved in FI. Indeed, it may be 
reasonable to assume that within a competitive/selective neural system (Edelman, 1993), the relative size of the neural population specifically involved in the given function is positively related to the individual's ability, although this remains to be demonstrated with respect to human FI and face processing in general. However, other factors contributing to EEG amplitude interindividual variability may be irrelevant to FI, such as the exact localization of the underlying neural populations with respect to cortical gyri and sulci and their orientation relative to the scalp, and interindividual variations in skull/scalp thickness (Herzmann et al., 2010; Luck, 2014). Hence, even though the FI function appears to be particularly well isolated in the present paradigm (see Section 5), variability in EEG amplitude across a homogenous population of neurotypical individual adults should not be expected to directly reflect variability in FI ability in this population.Despite these considerations, studies have reported significant, but relatively low, correlations between the FI response amplitude and the behavioral performance at tests such as the CFMT (accuracy; Xu et al., 2017) and the BFRT (response time; Dzhelyova et al., 2020). In our large-scale analysis with 130 individuals, the correlation with accuracy and the BFRT is quite weak $(r=0.25)$ but nevertheless highly significant ( $\mathrm{p}<0.005$; Figure 15). These weak correlations, which are not unlike correlations found between more traditional ERP responses to faces and behavior (e.g., N170 latency but not amplitude; Herzmann et al., 2010; Kaltwasser et al., 2014), should be interpreted carefully. At first glance, if behavior is considered as "the reference" measure, one would be tempted to conclude that the oddball FPVS measure with EEG does not capture FI ability very well. However, as discussed earlier in this review (Section 3.2), explicit behavioral performance at a (single) test such as the CFMT or the BFRT depends on many general processes beyond FI. Hence, behavioral tests of FI in the normal adult population are also only weakly correlated with one another (e.g., r=0.45 between the CFMT and GFMT in McCaffery et al., 2018; see also Stacchi et al., 2020 for weak to no correlations between several behavioral face recognition tests). Importantly, these weak correlations do not necessarily mean that the two measures (behavior/EEG, or behavior/behavior) reflect different FI processes (e.g., "face perception" vs. "face memory”). Instead, both measures 
may index the same core FI process, but are taken with different task requirements and thus differ in terms of the sources of general variations.
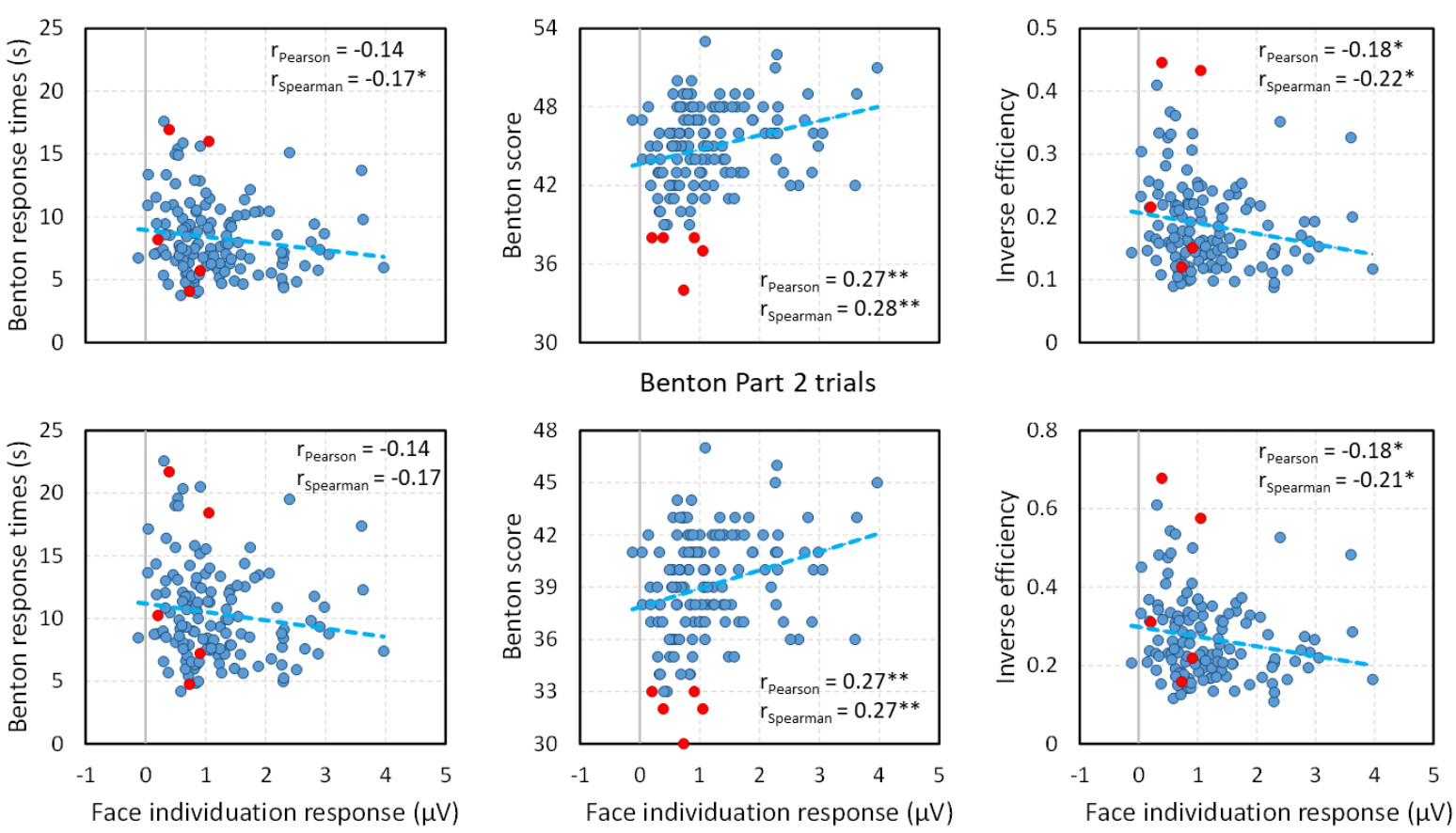

Figure 15. Correlations between the amplitude of the FI response with EEG and the Benton Face Recognition Test (BFRT) (large-scale analysis).

Correlations were run either on all 22 trials of the Benton test (top row) or only on the 16 trials of Part 2, in which faces needed to be matched across image changes (bottom row). This analysis included the 130 participants included in the group analysis, as well as 5 participants who were originally excluded due to low BFRT Scores ( $<39$ overall), shown in red. Relatively low but significant correlations are found between the EEG FI response and the BFRT in accuracy, RTs and inverse efficiency (see also Dzhelyova et al., 2020). The correlation with BFRT accuracy remains significant even after excluding the 5 low performers $\left(\right.$ rearson $=0.24 * *, r_{\text {Spearman }}=0.25^{* *}$ for both number of trials). $*: p<0.1, * *: p<0.01$ (two-tailed).

Our ultimate research goal is to understand human behavior in real life circumstances, yet there is no reason that a behavioral measure taken in a specific cognitive task in the laboratory (or on the internet; e.g., Germine et al., 2012) would be a "better" or "purer" reference measure of FI than the EEG measure reviewed here (see again Section 3.2). Instead, we have provided arguments supporting the view that the present oddball FPVS paradigm provides a highly valid measure of 
the FI function (Section 5.1), perhaps even more so than explicit behavioral tests. In other words, the weak correlation between the EEG FI responses with explicit behavioral performance in the normal population fails to undermine the functional validity of the FPVS FI measure, which is supported by many other lines of evidence (Section 5.1).

These considerations withstanding, what evidence is there that these weak but significant correlations between the FPVS FI responses and behavioral tests are genuine? If they were spurious correlations, there would be no reason to expect them to be higher for the FI EEG response at $F / n$ than for the stimulus presentation response at $F$, measured at its peak over medial occipital sites. Yet, the stimulus presentation response is uncorrelated with any of the BFRT variables ( $\mathrm{r}$ range $=$ $0.014-0.90, \mathrm{p}$-value range $=0.31-0.88)$. A careful look at correlation plots of the FI response with the BFRT indicate that the correlations are essentially driven by extreme individuals, in particular those with low accuracy and/or long response time (RT) and low amplitude. In the most recent study of Dzhelyova et al. (2020), the correlation between correct RTs at the BFRT and EEG failed to reach significance when three individuals with particularly low BFRT scores, who also happened to have the lowest EEG amplitudes for the FI response, were removed from the analysis. In our group analysis, the correlation may be particularly weak because all of the 130 included individuals had a BFRT score above 39, which is the cut-off score for the lower range normal performance, and which was used a criterion for participant exclusion (see Supplementary Material). Reintroducing the data of only 5 excluded individuals with a BFRT score below 39 slightly increases the correlations, both for accuracy and RT (Figure 15). This suggests that in a typical adult population, this paradigm may be particularly useful at rapidly identifying individuals who are poor at FI (i.e., below a certain threshold). Moreover, in a given clinical populations, where differences in ability may be more important between individuals, correlations between behavioral and EEG measures of FI might be increased. 


\subsection{The nature of the face individuation process}

With an advantageous technique in hand, one can start asking, or re-asking, key questions about the nature of the FI function.

\subsubsection{Shape and surface information}

Dzhelyova and Rossion (2014b) investigated the respective contribution of face shape vs. surface cues to FI in a design in which these two sources were independently varied at $F / n$. More specifically, the oddball face changed either in shape or surface cues, or both (see Figure 1 in that study). Face shape is essentially defined by the bone structure and soft tissue of the face, while surface cues reflect variations in color and texture (such as redness, stubble and wrinkles; Bruce \& Young, 1998). Results showed a large response over right occipito-temporal electrodes for FI based on in shape cues, but a more bilateral and larger response for FI based on surface cues, as supported by subsequent behavioral evidence (Itz et al., 2017). Critically, the sum of the FI response amplitude to shape and surface changes alone was lower than when both cues varied simultaneously, revealing a supra-additive effect not disclosed by behavioral studies (O'Toole et al., 1999; Russell et al., 2007; Itz et al., 2017). These observations suggest that the two kinds of cues combine nonlinearly during FI.

\subsubsection{Holistic individuation of faces through the composite face effect}

Identical top face halves appear perceptually different when aligned with bottom halves from different facial identities (Figure 16). This vivid perceptual illusion leads to strong behavioral effects ("the composite face effect") in FI tasks with pictures of celebrities (Young et al., 1987) or unfamiliar faces (Hole, 1994), thought to reflect the automatic integration of face parts (here the two halves) into a unified (i.e., holistic) representation ( Young et al., 1987). To date, more than 80 published studies have exploited this effect to examine the factors subtending holistic face recognition (for reviews see Rossion, 2013; Murphy et al., 2017), including its neural basis (Schiltz \& Rossion, 2006) and its temporal dynamics (Jacques \& Rossion, 2009). Nevertheless, as stated in 
a recent review, "the composite face illusion, whilst easy to illustrate, is deceptively difficult to measure and interpret" (Murphy et al., 2017, p.13). This is particularly the case in behavioral studies and has led to a number of controversies in the literature, most notably the claim that the composite effect has a decisional rather than a perceptual source (see Rossion, 2013).

A

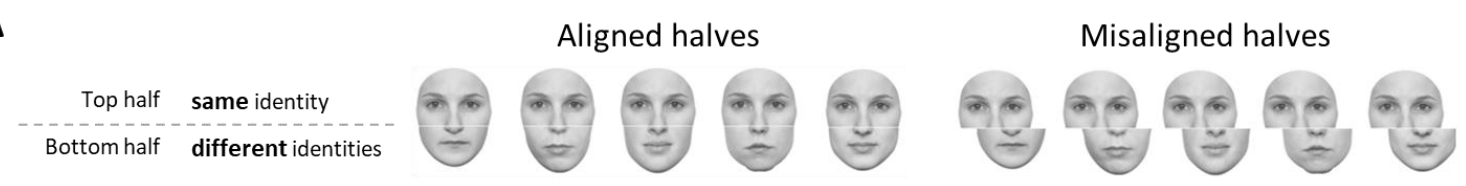

B

Identity
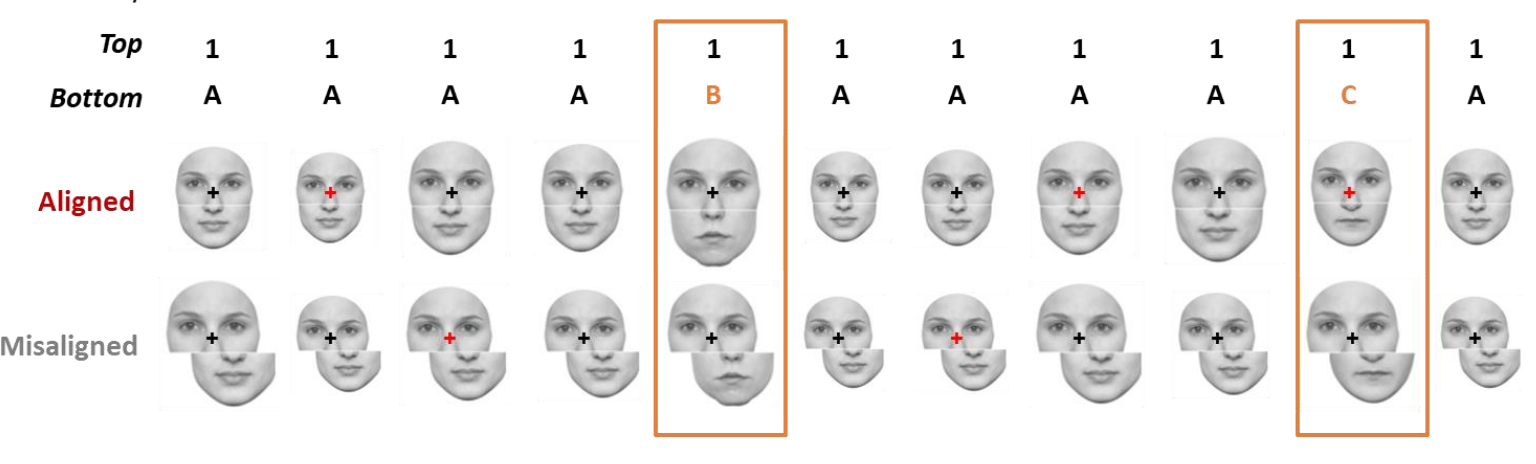

C
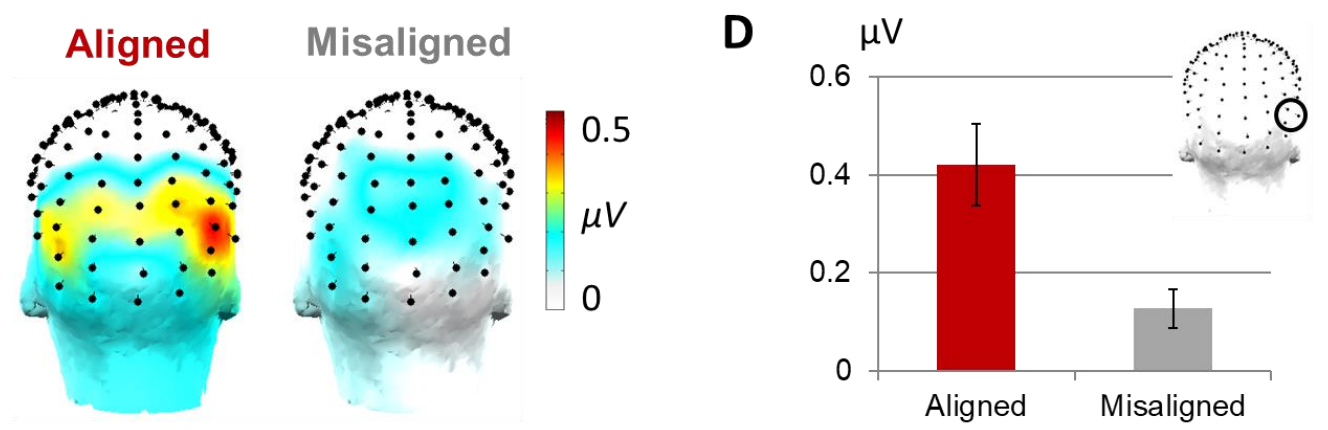

Figure 16. Composite effect (from Rossion et al., in preparation).

A. Example of composite face stimuli. The top half of one facial identity is paired with bottom halves from different unfamiliar facial identities, with a thin white space separating both halves (see Rossion \& Retter, 2015; these stimuli are available at: https://face-categorization-lab.webnode.com/resources/). In total there are 4 top half identities, each paired with 5 bottom half identities, for both aligned and misaligned conditions. B. Experiment design. In each minute-long sequence, a composite face with a randomly selected bottom half identity (e.g. 1A) was repeated as the base stimulus, with different bottom halves identities interspersed as oddballs at a $1 / 5$ interval (e.g. $1 \mathrm{~B}, 1 \mathrm{C} \ldots)(F=6 \mathrm{~Hz}, F / n=1.2 \mathrm{~Hz})$. Importantly, the identity of the top face half was always the same. Across sequences, composites faces were shown in either the aligned or the misaligned condition. Participants performed the usual orthogonal fixation cross task (color change detection). C. Back-view of the 3D scalp topographies of the FI scalp response in each condition. A strong right lateralized response is found to face identity change in the aligned condition, only. D. Mean amplitude 
of the FI response in each condition over a right occipito-temporal ROI. Misaligning face halves significantly reduced the FI response.

To overcome these complications, Rossion et al. (in preparation) measured FI with composite faces with 22 participants using the present paradigm (Figure 16). In this study, participants did not have to explicitly individuate faces, contrarily to a typical behavioral composite face paradigm. Instead, they simply fixated on the top halves of the face stimuli, which were presented at $6 \mathrm{~Hz}$, i.e., for the duration of one gaze fixation $(\mathrm{SOA}=167 \mathrm{~ms})$. When the bottom face half identity changed at $1.2 \mathrm{~Hz}$, a clear and strongly right lateralized oddball FI response was found, within a few minutes of stimulation. The response amplitude was much larger when face halves were aligned than when the halves were spatially misaligned, thereby revealing a neural composite face effect with this paradigm. Since no explicit decision-making regarding FI was required, these findings not only support the perceptual nature of the composite face effect, but demonstrate the capacity of the oddball FPVS paradigm to isolate it rapidly and objectively.

\subsection{Automaticity and task-modulation}

In the FI paradigm, participants usually complete a simple task orthogonal to the measured function, such as detecting rare, non-periodic changes of the fixation cross color (Figure 1). The robust responses found in these conditions indicate that individuation of unfamiliar faces is largely automatic, i.e., not under volitional control (Palermo \& Rhodes, 2007; Yan et al., 2017; see again Section 5.1.1). Nevertheless, it is interesting to evaluate how a face-related task would modulate the FI response in this context.

Yan et al. (2019) compared the FI response when participants performed either the usual orthogonal fixation task or an explicit face-related task (to detect randomly appearing male faces from among female faces; following Quek et al., 2018). Critically, both changes were concurrently present and occurred the same number of times within each sequence, such that visual input was constant and only attentional focus was varied. Results in the fixation task replicated previous 
studies, showing a strong FI response over the right occipito-temporal cortex. During the facerelated task, FI responses also peaked within the right occipito-temporal region, but were significantly increased across the entire scalp. To further examine this enhancement, EEG scalp topographies were normalized within each condition, revealing that when performing a face-related task, FI responses were disproportionately increased over prefrontal and right parietal channels, compared to the fixation cross task (Figure 17). Hence, while an explicit face task might increase absolute FI response magnitude (similarly to selective attention increasing ERPs and frequencytagged low-level responses, see Norcia et al., 2015 for review), it may also reduce the specificity of the response by recruiting additional cortical regions likely associated with general attentional and executive functioning, besides those typically associated with specialized FI (see Section 5.1.4).

A

Face presentation frequency : $F=6 \mathrm{~Hz}$

$\Rightarrow$ General visual response

Face identity change frequency : F/7 $=0.857 \mathrm{~Hz} \Rightarrow$ Face individuation response

\begin{tabular}{|c|c|c|c|c|c|c|c|c|c|c|c|}
\hline$A$ & A & $A$ & $A$ & $B$ & $A$ & $A$ & $A$ & $A$ & $c$ & $A$ & \\
\hline भ & ff & f) & 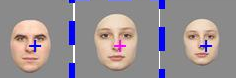 & $\vartheta$ & F & भ & भा बि 1 कि & 甲 & ? & $\%$ & $\begin{array}{l}\text { Fixation task } \\
\text { Detect fixation }\end{array}$ \\
\hline
\end{tabular}

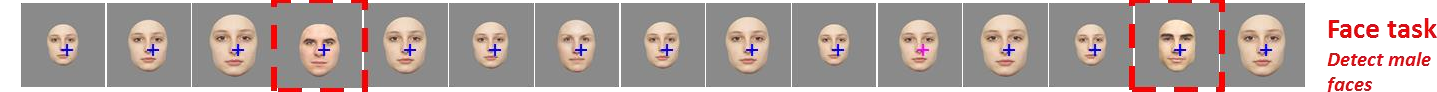

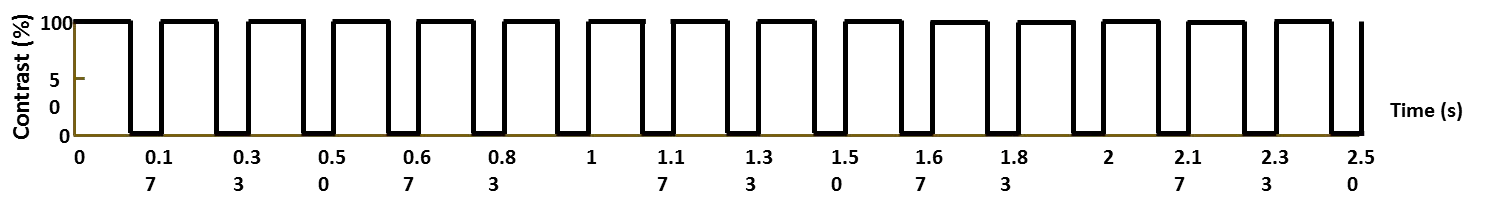

B

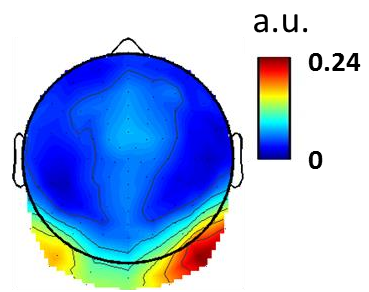

FIXATION

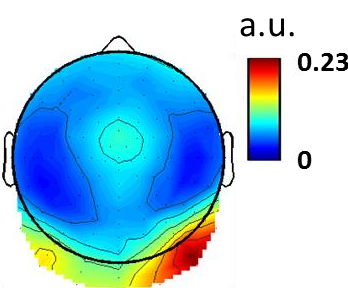

FACE

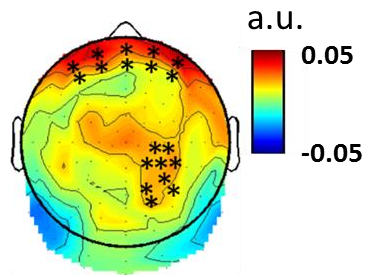

FACE - FIXATION

Figure 17. Effect of a face-related task on EEG face individuation responses (from Yan et al., 2019).

A. Schematic illustration of the experimental design. Here images were presented through squarewave contrast modulation ( $75 \%$ duty-cycle) at a fixed rate of $6 \mathrm{~Hz}$. The 70 s stimulation sequences contained different female faces $(B, C \ldots)$ interleaved at regular intervals (1/7) among a repeated identical female face 
(A). Two types of targets were present concurrently within each sequence: the central blue fixation cross turning red, and male faces replacing (oddball or base) female faces. Each of these target types randomly occurred 6 times within each sequence. Participants were given two different behavioral tasks in an alternated order: detect fixation color changes, or detect the appearance of a male face. B. Task modulation effects on topographically normalized FI responses. The asterisks on the difference map indicate channels reaching significance in a cluster-based permutation test and indicate relative topographical changes between tasks. Color scales are adjusted to the maximum value of each task.

\subsection{Application to typical \& atypical development}

\subsubsection{Typical development}

The typical development of the FI function is contentious. On the one hand, studies performed with newborns or infants often emphasize these observers' astonishing ability to individuate pictures of unfamiliar faces (e.g., Pascalis \& de Schonen, 1994; Scott, et al., 2006; Pascalis et al., 2002; Turati et al., 2008), going far beyond the recognition of a highly familiar face such as that of their mother's (Bushnell et al., 1989; Sai, 2005). ERP studies in infants also emphasize their early abilities at FI (e.g., Courchesne et al., 1981; de Haan et al., 2002; Scott et al., 2006; Righi et al., 2014; Peykarjou et al., 2014; Peykarjou et al., 2015; Adibpour et al., 2018). On the other hand, studies performed in young children, starting from 3-5 years of age, instead emphasize their low performance at behavioral FI tasks, with a progressive improvement noted until adolescence and adulthood (e.g., Carey, 1992; Sangrigoli \& De Schonen, 2004; Mondloch et al., 2006; de Heering et al., 2012; Hills \& Lewis, 2018; Mardo et al., 2018). This discrepancy is also found at the qualitative level: while effects of stimulus inversion on FI have been reported in newborns (Turati et al., 2006) and 3- to 4-month-old infants (Turati et al., 2004), this effect is absent or becomes less consistent in children of a few years of age (Sangrigoli \& de Schonen, 2004; Carey, 1981; Crookes \& McKone, 2009; de Heering et al., 2012). In a recent large-scale study, the 
behavioral face inversion effect has even been reported to emerge only around 8-9 years of age, and to increase progressively from then until adulthood (Hills \& Lewis, 2018) ${ }^{13}$.

Can these observations really be taken as evidence for the initial presence, subsequent loss, and recovery of FI ability throughout development? It appears more likely that the measurements of FI applied across development vary, and thus create discrepancies in their results. While infants are tested with implicit tasks, such as habituation-then-novelty-preference for one of two simultaneously presented visual items, or with ERPs, children from three years of age and adults are tested with explicit behavioral tasks, requiring matching/discriminating pictures of unfamiliar faces. That children's performance at these tasks appears limited relative to adults may well be due to task-related factors (task understanding, manual coordination, attention, motivation, visual search, short-term memory, decisional processes, etc.) that have nothing to do with FI ability per se (Crookes \& McKone, 2009; McKone et al., 2012). Moreover, children's performance is difficult to compare to that of adults due to differences in both accuracy and response time.

These issues of comparing across age groups are not easily resolved, as it makes little sense to test children and adults with visual preference techniques, and ERP studies in infants and children are particularly challenging due to the low SNR of the technique, which necessitates the recording and averaging of a large number of trials (Luck, 2014). Moreover, directly relating ERP findings between infants and children is difficult because the observed ERP components are markedly different. For instance, the P400 in infants is often related to the face-sensitive N170 in adults and children (e.g., de Haan et al., 2002; Halit et al., 2003) but the two components differ substantially in latency, polarity, and scalp topography, and do not share well-documented functional similarity, making this relationship implausible.

${ }^{13}$ While the authors claim that a key factor for inversion effects on FI performance is using faces of the same age as that of the participants (Hills \& Lewis, 2018), they do not provide any evidence supporting this proposition. 


\section{No Fl response in infants with this paradigm}

To resolve these issues and shed light on the development of FI, the present paradigm may be easily extended for testing across age groups. Indeed, a similar FPVS-EEG paradigm contrasting faces with objects at a fast $6 \mathrm{~Hz}$ stimulation frequency has been successfully used in 4- to 6-monthold infants, showing a specific neural response to highly variable natural images of faces as compared to equally variable images of objects (i.e., generic face categorization) (de Heering \& Rossion, 2015; Leleu et al., 2019; see also Peykarjou et al., 2017 for human vs. monkey face categorization in 9-month-olds). Yet, despite extensive testing, our group has so far been unable to find a reliable and significant FI response in 4- to 6-month-olds with the presently reviewed $\operatorname{paradigm}^{14}$.

How can we reconcile a lack of oddball FI responses in young infants with the clear evidence for FI in visual preference tasks? At first glance, it could be claimed that oddball FPVSparadigms with EEG are not sensitive enough to disclose FI in young infants. However, as repeatedly shown and highlighted in the present review, the oddball FPVS paradigm is highly sensitive, identifying significant responses at the individual level within $93 \%$ of neurotypical adults in a few minutes of testing only (Figure 12). Yet this paradigm is also challenging for observers, since it requires discrimination of multiple individual faces without external features, within a glance, and across substantial changes of image size. In comparison, habituation-then-noveltypreference studies often use only a few different face exemplars, present the probe images for a relatively long time, and often use strictly identical images for both the target and adaptor. Hence, evidence for unfamiliar FI in infants with this approach is seriously limited. Note that when using

\footnotetext{
${ }^{14}$ While Barry-Anwar et al. (2018) reported a small (right lateralized) FI response with this paradigm in 9month-olds for monkey faces, the physical differences between the base and oddball facial identities were particularly conspicuous, including differences in head orientation, and could explain the reported effect (see Fig. 1 in that study).
} 
so few stimuli presented in a pairwise fashion, even matching an unfamiliar facial identity across changes of head orientations (Turati et al., 2008; Kelly et al., 2009) could be easily achieved based on low-level visual cues (e.g., amount of white in the sclera, lip thickness, etc.; see Turati et al., 2008, Fig.2).

As for standard ERP studies using repetition of faces in infants, their results are generally inconsistent (e.g., no effect in Peykarjou et al., 2014 in 9 month-olds; effect on a N290 component for female face pictures only in Righi et al., 2014, but a later effect for male face pictures only in Peykarjou et al., 2015), in line with the low test-retest reliability of stimulus manipulations on infant ERP components (Munsters et al., 2019). Moreover, since they rely on the repetition of the exact same images, the effects reported in some studies could also be attributed to differences in global luminance and contrast (see also Adibpour et al., 2018). The localization of the largest ERP effects on medial occipital channels for central stimulus presentation (e.g., channel Oz in Peykarjou et al., 2015) supports this interpretation.

In summary, while it could be argued that the FI function in infants should be probed with more simplified approaches than the present paradigm, we rather favor the view that it is important to measure the FI function with the exact same approach across all ages. So far, there is no convincing evidence with the present paradigm that the FI function - with unfamiliar faces, devoid of external features, at least - is already present in young infants, contradicting behavioral and (some) ERP studies. To illustrate this position, it may be useful to draw a parallel to observations made in prosopagnosia. Despite her profound impairment at FI in real life, the patient PS is able to individuate pictures of unfamiliar faces well above chance level when she is not put under severe time pressure (e.g., Rossion et al., 2003; Busigny et al., 2010). In fact, a number of patients with (acquired) prosopagnosia can reach normative levels of performance in terms of accuracy at explicit unfamiliar face matching tests (Benton \& Van Allen, 1972; Davidoff \& Landis, 1990; Farah, 1990; Delvenne et al., 2004), and such patients would undoubtedly be able to discriminate individual face images in infant visual preference paradigms. However, when tested in the FPVS 
FI paradigm, patient PS showed no FI response at all (Liu-Shuang et al., 2016; Figure 9, Section 5.2.1). Such observations suggest that this paradigm does not trade sensitivity for specificity, and that the absence of a significant response in this paradigm for infants - at least before any convincing evidence is reported - indicates the immaturity of their unfamiliar FI abilities.

\section{A FI response in five-year-old children with this paradigm}

In five-year-old children, a clear FI response at $F / n$ is present over the right occipitotemporal cortex, as for adults (Lochy et al., 2020; Figure 18). Note that a clear right lateralization is present here before formal reading acquisition, thus contradicting the hypothesis that the right lateralization for faces results from a competition with letter and word representations during formal reading acquisition (Behrmann \& Plaut, 2013; Dehaene et al., 2015). However, there are major difference between the children's FI response and the adults' FI response. First, while children's FI response amplitude is quite high (similarly as with ERPs in children, e.g., Kuefner et al., 2010), it is much lower in SNR (amplitude relative to background EEG noise) than for adults, and its amplitude relative to that of the general visual response at $F$ is smaller than in adults. Second, for children, the first harmonic of the oddball frequency $(F / n)$ accounts for the bulk of the FI response (about 60\%, Figure 18A, compared to only $18 \%$ in adults, Figure 4), suggesting a simpler or longer response (Lochy et al., 2020 see Box 1). Third, strikingly, there is almost no reduction of the FI response with picture-plane inversion in children (i.e., a mere $11 \%$ amplitude decrease, just reaching significance in a large sample; Lochy et al., 2020). Interestingly, in an older population of 8- to 12-year-olds, the EEG responses are much more adult-like, with a large and complex FI response distributed over many harmonic frequencies, and a large inversion effect (about 50\%; Figure 18B; Vettori et al., 2019b). 

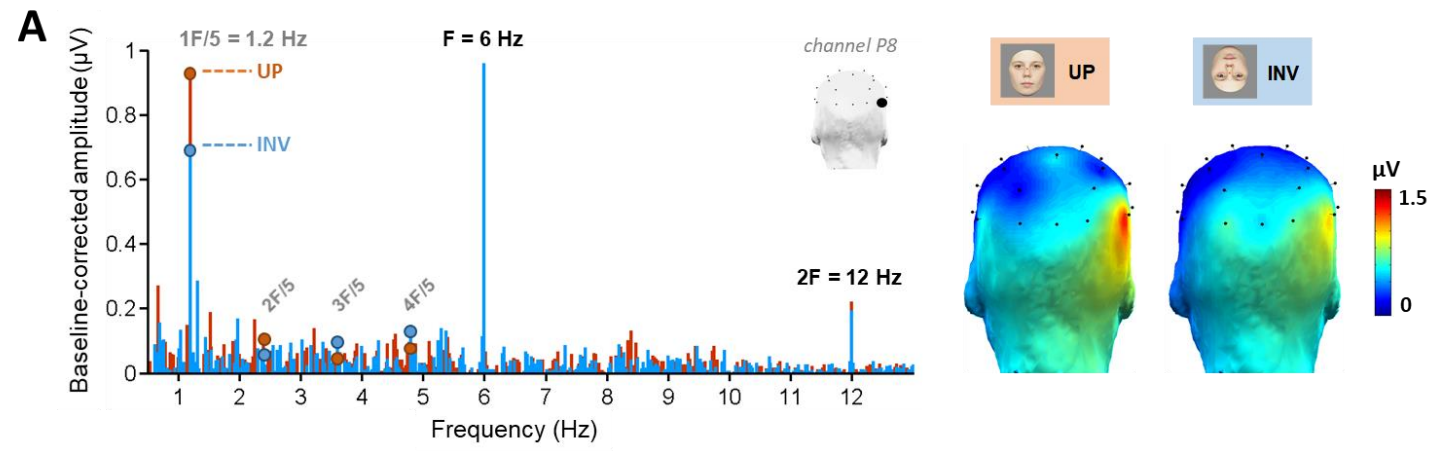

B
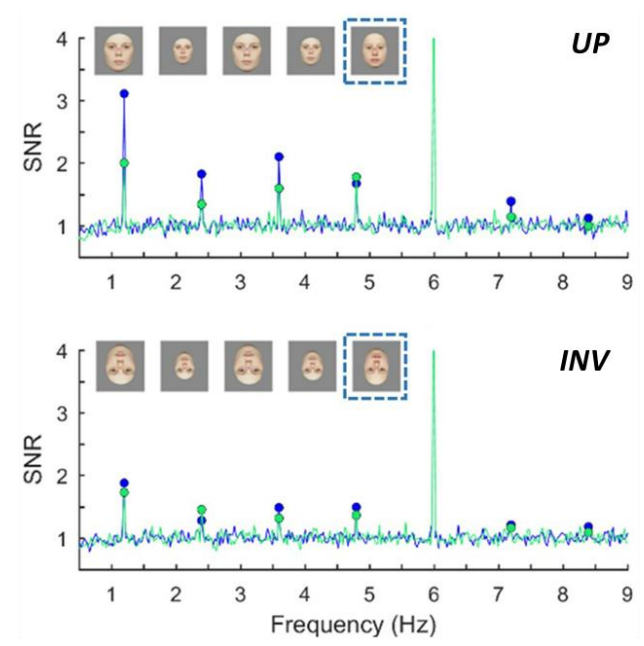

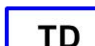

ASD
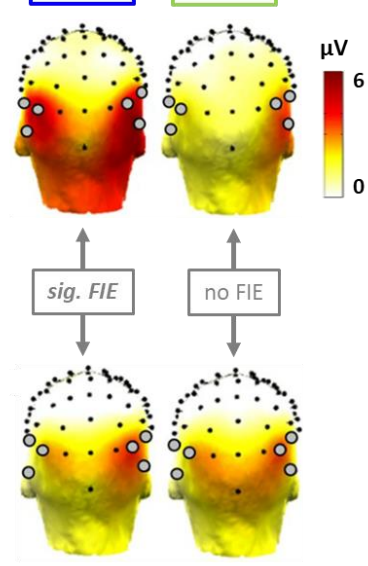

TD - ASD
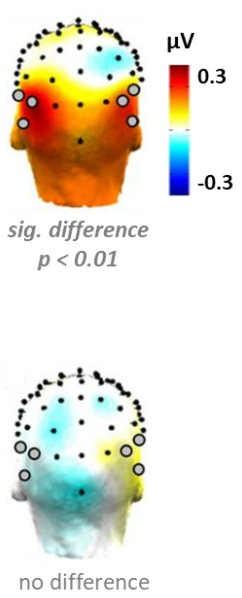

Figure 18. Typical and atypical development of FI (from Lochy et al., 2020).

A. Typical development. The amplitude spectrum shows responses obtained in 5-year-old children (preschoolers) in the oddball FPVS paradigm. Large peaks at $F$ and its harmonics are found for the general visual response $(6 \mathrm{~Hz}, 12 \mathrm{~Hz}$, etc.). However, the $F / n$ FI response is predominantly distributed only over the first harmonic $(1.2 \mathrm{~Hz})$, contrarily to adults (Figure 4). Additionally, the children's FI response is much less reduced with face inversion (11\%) compared to older children (8-12 years old, Figure 18B) and adults (Figure 7). B. Atypical development (from Vettori et al., 2019b). The oddball FPVS paradigm reveals multiple dissociations in the FI response from 8- to 12-year-old boys that are either typically developing (TD) or with autism spectrum disorder (ASD). The FI response for upright faces is significantly larger in the TD than the ASD group (see the subtraction: TD - ASD), but the groups do not differ in response to inverted faces. Moreover, only the TD group show a significant face inversion effect. Data from the frequency spectra are taken from a bilateral occipito-temporal ROI shown on the 3D scalp topographies on the right panel.

Altogether, these observations refute the view that the FI is fully mature early in development (i.e., by 5 years of age) and that all subsequent improvement on experimental task performance results from the development of other, general factors (Want et al., 2003; Crookes \& 
McKone, 2009; McKone et al., 2012). Instead, these results indicate that the FI function undergoes substantial and specific development, with major changes occurring between 5 and 12 years of age, perhaps linked to an increased exposure to the large number of faces encountered in school environments. This is in agreement with neuroimaging investigations showing an increase in faceselective responses in the fusiform gyrus between 7 years of age to adulthood (Golarai et al., 2007; Scherf et al., 2011; Golarai et al., 2017) and more generally with a face-specific recognition development theory (e.g., Carey \& Diamond, 1977; Mondloch et al., 2002). What is currently missing however, is a systematic evaluation across development with the same paradigm, with both upright and inverted faces, to track the emergence and the evolution of the FI response from birth through adulthood.

\subsubsection{Atypical development}

\section{Autism Spectrum Disorder}

Increased knowledge about the typical development of the FI function is important in itself, but also serves to better characterize the atypical development of this function. In particular, deficits in face processing have been put forward as a hallmark of social difficulties in Autism Spectrum Disorder (ASD; American Psychiatric Association, 2013) and many studies have tested individuals with ASD on explicit behavioral face processing tasks (Tang et al., 2015 for review). Early studies showed that young children with ASD were less proficient than neurotypically developing controls at identifying familiar peers based on the eye region, and had a smaller face inversion effect (Hobson et al., 1988; Langdell, 1978). However, findings from numerous behavioral studies testing face processing in ASD are generally inconsistent, with some studies reporting poorer face processing abilities in ASD (e.g., Tantam et al., 1989; Rose et al., 2007; Rosset et al., 2008), and others reporting similar performance to neurotypical individuals (e.g., Jemel et al., 2006; Reed et al., 2007; Scherf et al., 2008). Again, comparisons across studies are difficult due to the use of different populations (e.g., in terms of age, gender, and intelligence) and 
the vast heterogeneity in ASD inclusion criteria, but also because of large differences in task requirements. For instance, children with ASD may be able to perform individual discrimination tasks with simultaneously presented faces, but be impaired when faces are shown consecutively (Weigelt et al., 2012). This discrepancy may not be caused by selective impairments in "face memory" as opposed to "face perception" (which are usually poorly defined concepts that cannot be directly applied to complex behavioral tasks), but rather more simply to the fact that FI is more difficult and involves more general cognitive processes when stimuli cannot be compared side-byside (see also Powell et al., 2019). While standard face-related ERPs, in particular the N170, have been measured in children or adults with ASD, these studies have failed thus far to provide consistent evidence for abnormalities of the N170 component in ASD in terms of amplitude, latency or scalp topography (Kang et al., 2018; Key \& Corbett, 2020). This is understandable given that these parameters of the N170 do not directly reflect the FI function but mainly the visual response to the sudden presentation of faces (see Vettori et al., 2019a).

Two recent studies have used the present paradigm to measure FI in individuals with ASD. Dwyer et al. (2019) tested 16 individuals with ASD and found no differences with a typically developing group in the amplitude of their FI response. In contrast, Vettori and colleagues (2019b) found substantially reduced FI responses over the occipito-temporal cortex in a group of 23 ASD boys compared to typically developing controls (Figure 18b). This difference vanished when faces were inverted, due to the lack of a significant face inversion effect in the ASD group. Moreover, general neural responses to the visual stimulation at $6 \mathrm{~Hz}$, and neural responses indexing generic face categorization (i.e., faces $v$ s. objects) were indistinguishable between children with ASD and typically developing controls.

While the discrepancy between studies may not appear to be encouraging at first glance, there were major differences between the two studies. Dwyer et al. (2019) tested adults, both male and female, with a variable age range. Most importantly, the ASD group was comprised of selfselected individuals who reported themselves as having a diagnosis of ASD, but without any formal 
clinical assessment. In contrast, Vettori et al. (2019b) tested male children in a limited age range, who were formally diagnosed with ASD. The study of Vettori et al. (2019b) was also collected on a larger sample, and with more control conditions (Figure 18B). Hence, while it could be that a deficit of the FI function in ASD children is no longer observed in adulthood with this paradigm, the data of Vettori et al. (2019b) provide substantial evidence for a selective high-level impairment at FI in ASD. Providing that such observations are strengthened in the years to come, the objective and rapid assessment of FI, as well as its high test-retest reliability, may open new perspectives for contributing to the characterization and identification of disorders such as ASD, with implications for clinical diagnoses (Rossion, 2020).

\subsection{Time-domain information}

Contrary to standard ERP studies, the primary aim of the oddball FPVS paradigm with EEG is not to investigate the temporal dynamics of FI, i.e., when do neural responses occur, for how long, and with what temporal characteristics. However, providing that adequate stimulation parameters are used, meaningful information about the time-course of FI responses can be extracted with this paradigm. 
A
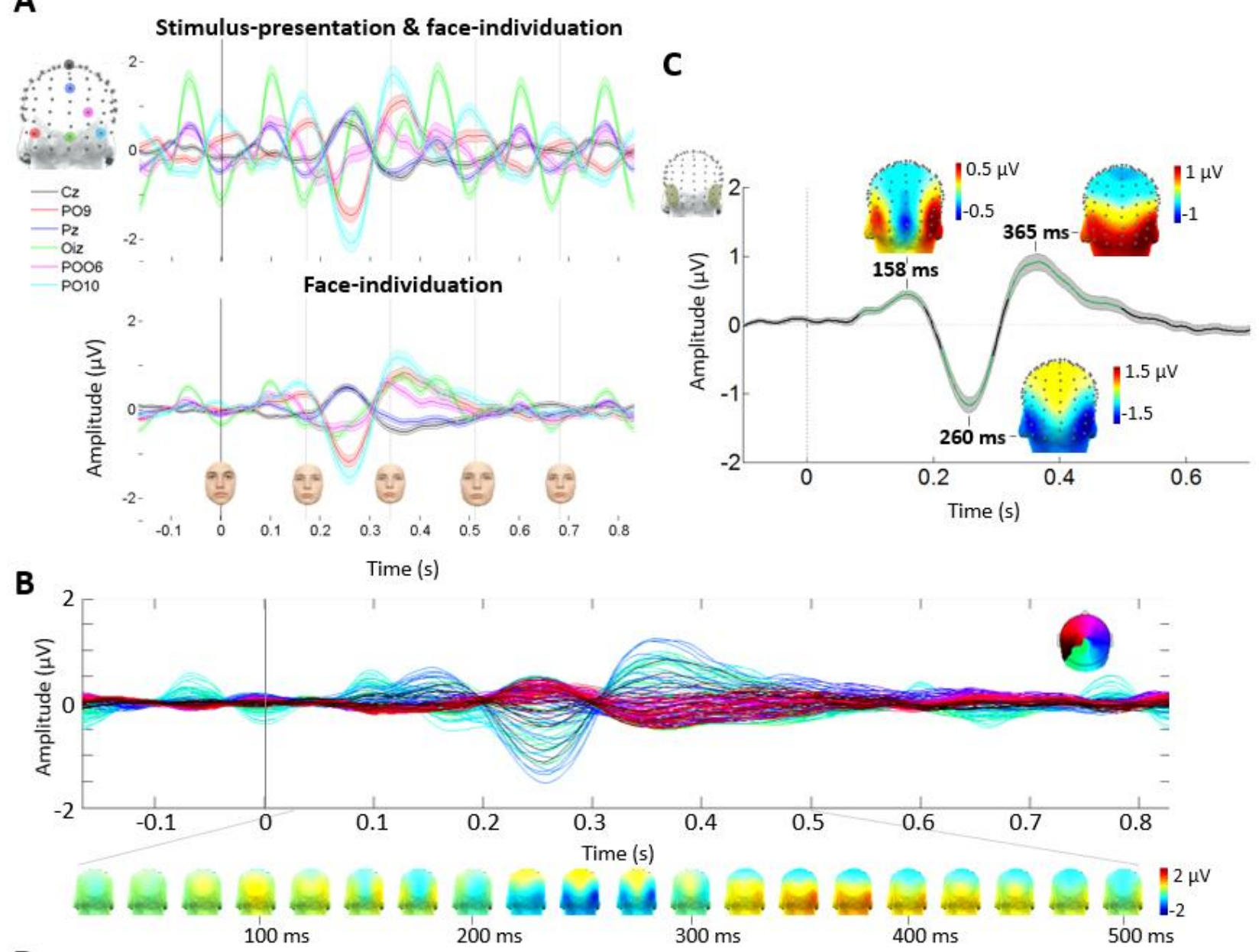

D
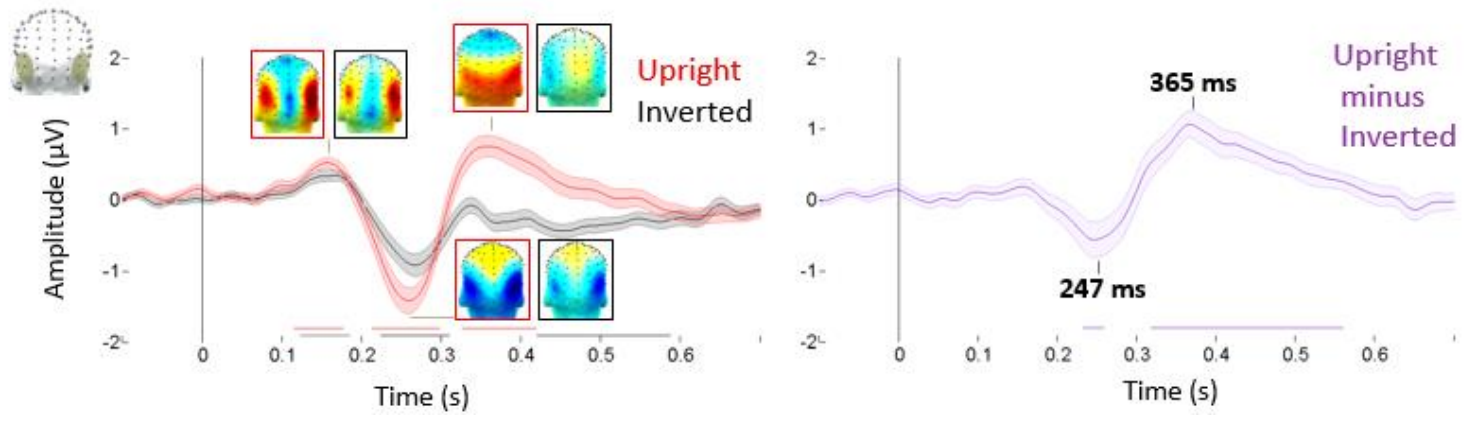

Figure 19. Time-domain FI responses (large-scale group analysis).

A. Responses in the time domain, relative to oddball identity changes at $0 \mathrm{~s}$. Responses to both FI at $F / n$ and stimulus presentation at $F$ are apparent in non-notch filtered data (top panel). After selectively removing the stimulus-presentation responses (with a FFT notch filter at $F$ and its harmonics; see Supporting information for methods), FI responses are isolated. Light gray bars indicate the time of additional base face stimulus presentations, occurring at $F$. The shaded area indicates one SE. B. The isolated FI responses are plotted here across all 128 EEG channels. 3D scalp topographies (back of the head) are plotted every 25 $\mathrm{ms}$, from $25 \mathrm{~ms}$ to $500 \mathrm{~ms}$. C. A simple characterization of the isolated FI response over a bilateral occipitotemporal ROI. The response is characterized by an early positive deflection peaking at $158 \mathrm{~ms}$ post stimulus onset (through sinusoidally modulated image contrast, beginning at $0 \%$ contrast), following by a large negative deflection peaking at $260 \mathrm{~ms}$, and then a late positive deflection peaking at $365 \mathrm{~ms}$, persisting until 
$500 \mathrm{~ms}$. Significant deflections ( $\mathrm{p}<.01)$ are colored in green, and the shaded area indicates one SE. D. Left: A comparison of isolated FI responses to upright (red waveform) and inverted (black waveform) faces. 3D scalp topographies reflect the peak deflection times identified in Panel C. Significance is indicated by respectively colored bars below the waveforms; the shaded area reflects one SE. Right: A subtraction of the inverted from the upright FI responses, as shown in the left panel. Significant differences, indicated with lines below the waveform, are found only at the time of the negative and late positive deflections.

\subsubsection{How to capture temporal dynamics with this paradigm}

In EEG frequency-tagging paradigms, time-course information can sometimes be reconstructed by analyzing the frequency-domain phase information of a dominant first harmonic response (e.g., Apppelbaum et al., 2006; see Norcia et al., 2015). However, here the $F / n$ FI response occurs at a relatively low frequency and its amplitude is distributed over multiple harmonic frequencies (Figures $3 \&$ 4). Each harmonic frequency has its own phase value, and it remains unclear how such phase information should be interpreted collectively, apart from amplitude. A more straightforward way of proceeding is to apply a standard ERP analysis to the FPVS EEG data in the time-domain: each facial identity change in a sequence is considered an event, and time epochs are segmented around each event (e.g., -100 ms to $600 \mathrm{~ms}$ ) and averaged together to obtain the temporal waveform of the FI response (Figure 19). The resulting signal is dominated by a series of large deflections at the stimulus presentation frequency $F$ (e.g., a $6 \mathrm{~Hz}$ "oscillation"), such that the exact onset and offset of responses specific to the changes of facial identity are difficult to identify. Fortunately, the frequency of $F$ is known, and is isolated within a single frequency bin from the rest of the EEG frequency-domain spectrum (see Section 4). Thus, $F$ and its harmonics can be selectively removed through frequency-domain notch-filtering, prior to event segmentation (Figure 19A; see Dzhelyova et al., 2014b and Yan et al., 2019 for additional examples of response waveforms with and without removing $F$ ).

\subsubsection{Insights into the temporal dynamics of face individuation}

The above procedure results in a series of well-defined EEG deflections over the occipitotemporal cortex selectively evoked by facial identity changes in the sequence (Figure 19B; see also 
Dzhelyova et al., 2014b; Yan et al., 2019). Importantly, these deflections essentially reflect differential processing between the common responses to all face stimuli (which have been selectively eliminated) and responses to identity changes, and should not be equated with standard ERP components elicited by face stimuli (e.g., P1, N170, etc.).

The first deflection, over occipito-temporal channels, is positive, peaking at about $160 \mathrm{~ms}$; it is followed by a large negative deflection, peaking at $260 \mathrm{~ms}$; and the response finishes with one or two additional positive deflections (first peaking at $365 \mathrm{~ms}$ ) also prominent over occipitotemporal sites (Figure 19C). Notably, there is no evidence of a parieto-centro-frontal generic P300 (Sutton et al., 1965; Picton, 1992), or subcomponents P3a or P3b, in this FI oddball paradigm, in which there is no active detection of the periodic changes. Moreover, the FI response is complex and cannot be reduced to a single generic response such as a visual mismatch negativity (vMMN) occurring to violations of regular sequential patterns between 200-400 ms (Kimura et al., 2011).

One limitation in assessing the temporal dynamics of the FI responses here is that the studies of the large-scale group analysis presented stimuli with sinusoidally modulated contrast, such that the image contrast was actually $0 \%$ at the image onset time $(0 \mathrm{~ms})$ : contrast increased sinusoidally, such that the face was likely only perceptible (above about $30 \%$ contrast) between about 30 to $140 \mathrm{~ms}$ of stimulus presentation time, with a stimulus presentation rate of $5.88 \mathrm{~Hz}$ on a $100 \mathrm{~Hz}$ display refresh rate. Thus, the responses are likely delayed by about $30 \mathrm{~ms}$ with this presentation mode, relative to an abrupt stimulus onset (see Retter \& Rossion, 2016; Dzhelyova et al., 2017 for examples contrasting response latencies following sinusoidal vs. abrupt presentation FPVS modes with generic face categorization and emotion; see also Retter et al., 2018 for a comparison of $50 \%$ vs. $100 \%$ squarewave stimulus contrast modulation, reporting a delay of about $20 \mathrm{~ms}$ for the latter).

A second limitation in characterizing the temporal dynamics of the FI response with the oddball FPVS paradigm is that there may be intermodulation between the stimulus-presentation $F$ response and the $F / n$ response. If that were the case, even after removing the $F$ response with 
frequency-domain filtering, the $F / n$ response would remain affected (note that most relevant intermodulation frequencies, e.g., $F+F / n, F-2 F / n$, etc., coincide with harmonics of $F / n)$. Such interactions might be expected because: 1) both the $F$ and $F / n$ responses are elicited from face stimuli, and so likely have common high-level neural sources; and 2) a stimulus presentation rate of $F=6 \mathrm{~Hz}$ is typically used (and indeed, $F$ between 4 to $6 \mathrm{~Hz}$ provides the largest FI responses: Figure 20), but $F$ thus falls into a range of frequencies typically containing high amplitude $F / n$ harmonic frequency responses (Figure 4). For empirical hints of potential non-linear interactions between $F$ and $F / n$ responses in the time domain, see baseline (pre-oddball stimulus onset) activity in the filtered waveforms in Figure 19A, which coincides with base stimulus presentation responses in the unfiltered waveforms. However, the $F / n$ FI response does not appear to have a markedly different onset latency, or otherwise different temporal dynamics, when $F$ is varied (Figure 20D; Retter et al., in preparation), and stable FI response amplitudes are observed as $F$ is varied from 4 to $6 \mathrm{~Hz}$ (Figure 20B).

This last limitation may be particularly relevant when considering the onset of FI responses recorded in this paradigm: in the large-scale group analysis, the FI response ranges from about 90 $\mathrm{ms}$ to $500 \mathrm{~ms}$ over occipito-temporal channels, i.e., extending for a total duration of about $410 \mathrm{~ms}$ (Figure 19C). FI response onset latencies around $100 \mathrm{~ms}$, or even earlier, have been reported in previous oddball FPVS studies: significant at $110 \mathrm{~ms}$ with $5.88 \mathrm{~Hz}$ sinusoidal stimulation, without stimulus size changes, in Dzhelyova et al., 2014b; apparent before $100 \mathrm{~ms}$ with $6 \mathrm{~Hz}$ sinusoidal stimulation, with a modified design, in Fig. 6 of Feuerriegel et al., 2018; described from $\sim 80 \mathrm{~ms}$ with squarewave stimulation in Yan et al., 2019; significant at $\sim 75 \mathrm{~ms}$ with continuous stimulation in Retter et al., in preparation: see Figure 20D). Should it be concluded that high-level FI occurs at $90 \mathrm{~ms}$ (or even $60 \mathrm{~ms}$, if roughly accounting for the sinusoidal stimulation onset delay)? This very early FI response onset is likely better attributed to low-level adaptation/repetition suppression to the base face, as mentioned above. Indeed, it appears to begin with medial occipital activity that rapidly transfers towards occipito-temporal regions (see Figure 19A\&B), with negative medial 
occipital activity being present at the time of the occipito-temporal response peak (Figure 19C). Moreover, unlike later response deflections, this early response appears to be affected by low-level image properties, such as stimulus size (see Figure 4 of Dzhelyova \& Rossion, 2014a) and head orientation (Or et al., in preparation) and, most importantly, is not significantly larger for upright than inverted faces (Figure 19D).

Note that despite decades of research on the temporal onset of FI responses with standard EEG/MEG stimulation modes (i.e., since Barrett et al., 1988), results remains equally ambiguous, with some studies claiming that it starts at the level of the N170 peak (Rossion \& Jacques, 2011 for review), others rather identifying this onset latency well after 200 ms (i.e., N250r, Schweinberger \& Neumann, 2016 for review; see also Endnote 4), and yet others emphasizing very early "face identity decoding" responses (<100 ms), undoubtedly related to image-based cues (e.g., Nemrodov et al., 2016; Vida et al., 2017; Dobs et al., 2019).

Here, what is clear is that there are multiple deflections over time reflecting the FI process. Importantly, even though sensitivity to different face properties may emerge at various points in time over these deflections, we are adamant that they should not be referred to as independent "stages" of "components" but rather reflect a progressive accumulation of evidence for face individuation. 


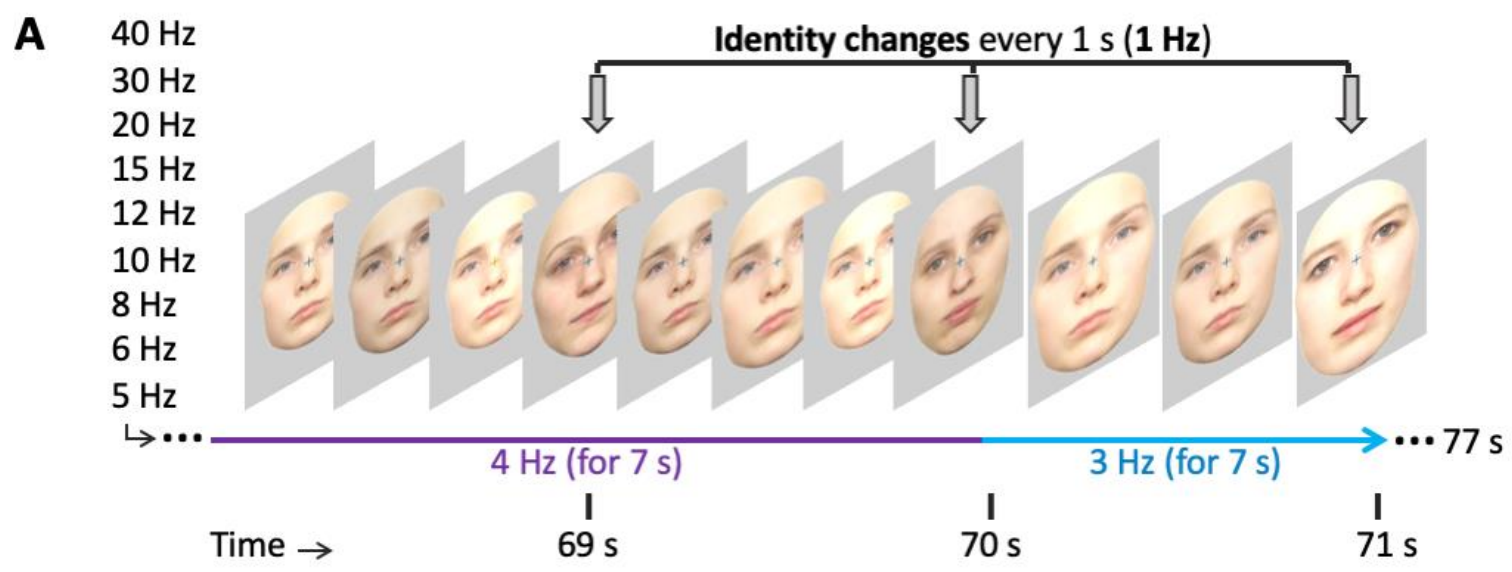

B Identity-change: $1 \mathrm{~Hz}$ +harmonics

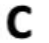

Scalp topographies
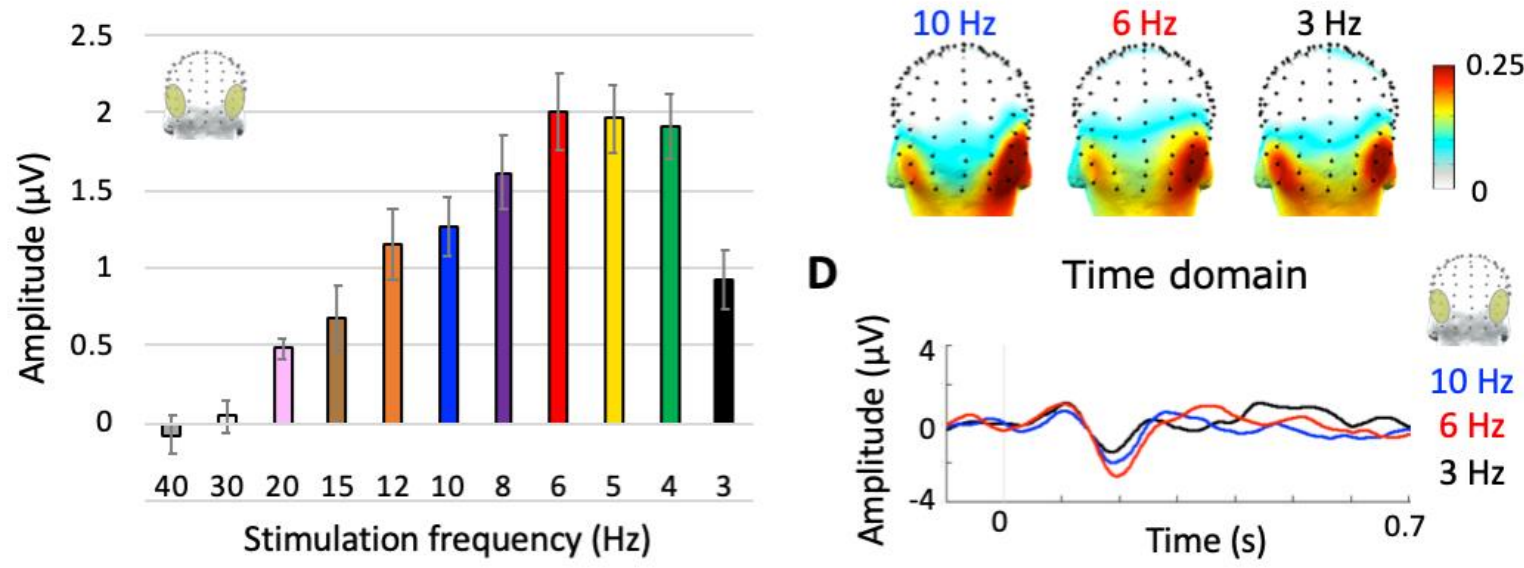

Figure 20. Speed of FI (from Retter et al., in preparation.

A. Oddball FPVS frequency-sweep paradigm: the stimulus presentation frequency $F$ is swept from 40 to 3 $\mathrm{Hz}$ in nine 7-s steps; despite changes in $F$, the identity change frequency $F / n$ was held constant at $1 \mathrm{~Hz}$. B. FI responses, measured by the sum of specific $F / n$ harmonic frequency amplitudes from 1 to $20 \mathrm{~Hz}$, over the indicated occipito-temporal ROI. The largest FI response occurred when $F=4$ to $6 \mathrm{~Hz}$. C. Qualitatively similar FI response scalp topographies (normalized here) were produced across presentation rates, despite large amplitude differences. D. An analysis in the time-domain, to identity-changes occurring at $0 \mathrm{~s}$, also revealed qualitatively similar responses in the first positive and negative deflections, and confirmed the largest FI response amplitude at $6 \mathrm{~Hz}$ reported in the frequency domain.

Understanding the temporal dynamics of FI has implications for the neural bases of this process, as well as practical implications for the speed at which it may be carried out. The duration of the FI response, being over $400 \mathrm{~ms}$ at occipito-temporal channels (Figure 19C), suggests that FI response interruption may occur at $F / n$ rates of about $2.5 \mathrm{~Hz}$ or greater. Why $F / n$ and not $F$ ? Let us consider the simpler case in which FPVS sequences of repeated $v$ s. different facial identities are shown. In this case, right occipito-temporal response topographies were observed when presenting 
FPVS sequences of a repeated facial identity at 1 and $2 \mathrm{~Hz}$, but responses shifted to become more medial at $3 \mathrm{~Hz}$ and above (Alonso-Prieto et al., 2013). That is, as stimulus presentation rate increased, identity-specific responses may have produced interference with one another and have been reduced (see again Section 6 and Box 1), producing relatively larger low-level activity to stimulus presentation. In contrast, the responses to FPVS sequences of different facial identities in that study continued to produce dominant right occipito-temporal responses until near $10 \mathrm{~Hz}$, in agreement with the range of occipito-temporal FI harmonic frequency responses reported here earlier (Figure 4). These results suggest that there is competition in the neural representations of a repeating facial identity (e.g., both producing neural signature A), but less competition in the neural representations of different facial identities (e.g., one producing neural signature A, and the other producing a neural signature $\mathrm{B}$, that is at least partially distinct).

In the present paradigm, oddball identities are typically separated by more than $800 \mathrm{~ms}$, such that they are unlikely to interfere with one another. Instead, as discussed above (Section 6), it is likely that the repeated base face responses do interfere with one another, contributing to the RS effect. While response amplitudes were decreased dramatically above $2 \mathrm{~Hz}$ in the study by AlonsoPrieto et al. (2013; see Figure 7 of that study), responses were more reduced for same $v s$. different facial identity FPVS sequences up to about $7 \mathrm{~Hz}$. Does this mean that faces cannot be individuated at or above $8 \mathrm{~Hz}$ ? This extreme statement is not necessary to describe the results: it is enough that faces cannot be individuated consistently at or above $8 \mathrm{~Hz}$. Indeed, in the study of Retter et al. (in preparation), when $F / n$ was held at $1 \mathrm{~Hz}$, and $F$ was swept from 40 to $3 \mathrm{~Hz}$, a progressive decrease of amplitude at $8 \mathrm{~Hz}$ and above suggests that progressively fewer faces were individuated at these higher rates, but enough FI to produce a significant EEG response was found up to $F=20 \mathrm{~Hz}$ (Figure 20; see also Retter et al., 2020). 


\section{Methodological guidelines}

Despite its simplicity, the paradigm reviewed here provides reliable and robust, focal FI responses over the occipito-temporal cortex that can be objectively identified and quantified within a few minutes. Yet, it is not panacea, and in addition to the above considerations regarding the interpretation of temporal dynamics, there are a number of important issues to consider when running this paradigm.

\section{EEG acquisition}

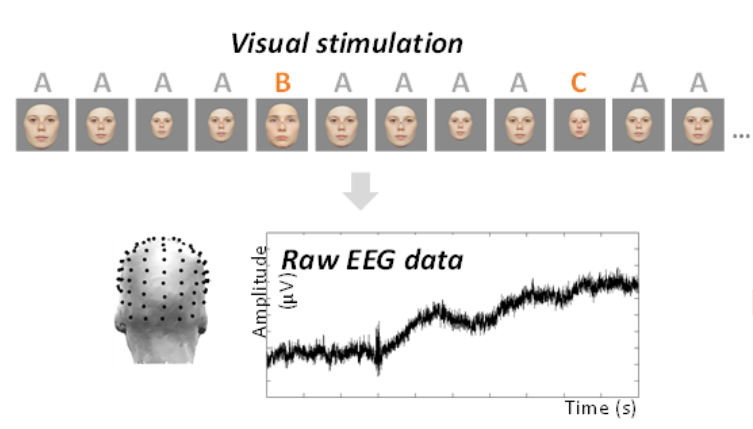

\section{Response quantification}

Segment \& sum amplitude sections (excluding image presentation frequency harmonics)
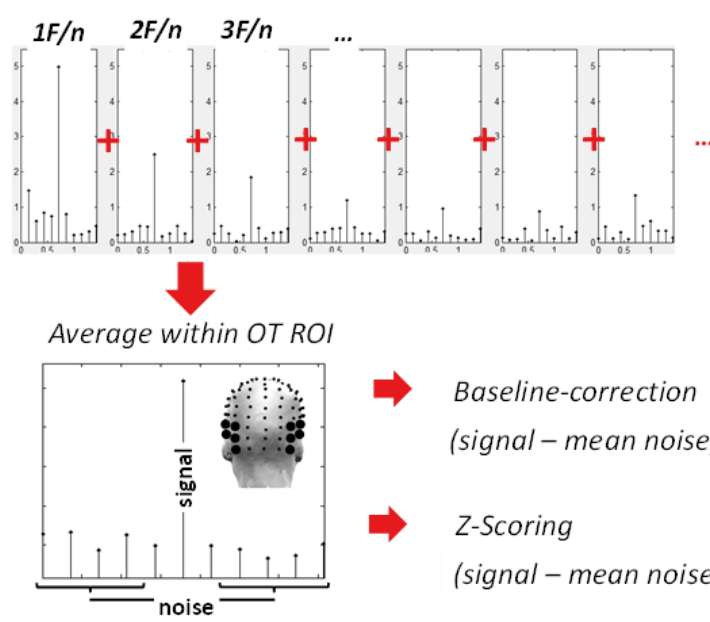
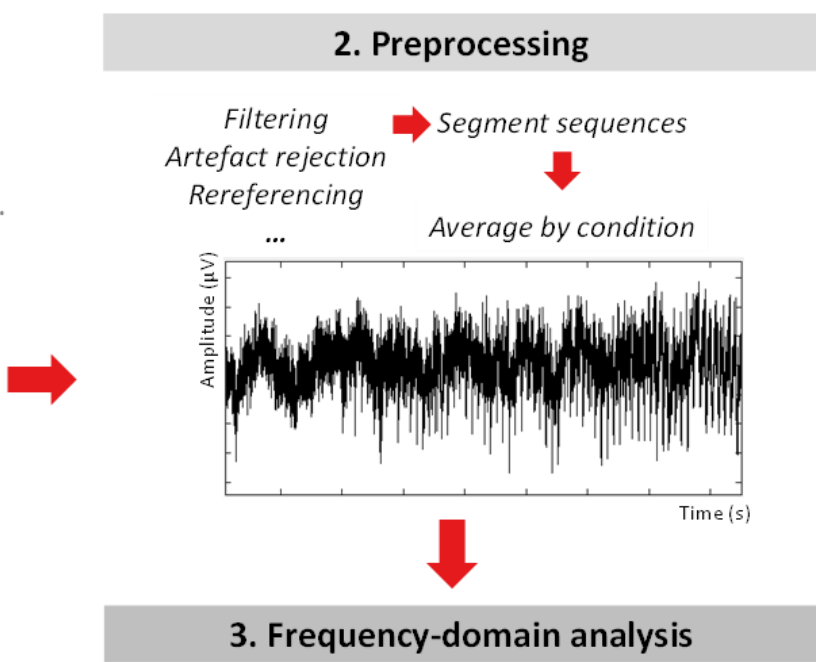

Frequency-domain analysis

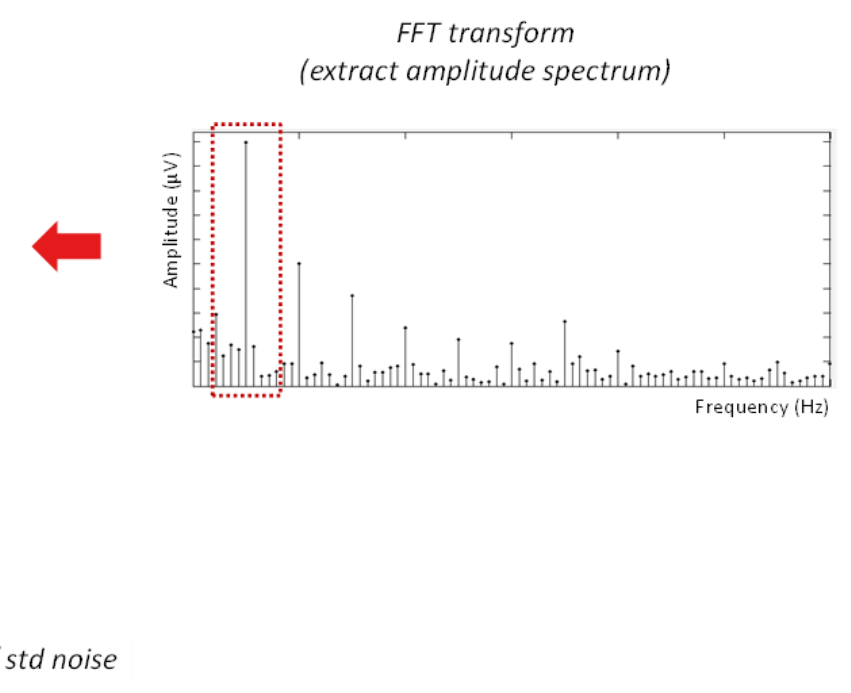

Figure 21. General data analysis procedure. Following (1) data acquisition, the raw EEG data is usually (2) preprocessed (i.e., low/high pass filter; re-reference; blink removal with independent component analysis ...) and segmented to contain only the relevant stimulation duration with an integer number of oddball frequency cycles. These epochs are then averaged by condition/subject and (3) transformed into the frequency-domain with FFT. From the resulting amplitude spectrum, (4) "chunks" containing the relevant 
oddball frequency harmonics (representing signal) and neighboring bins (representing noise) are extracted and summed. The selection of the relevant harmonics can be performed with a Z-score transform to select only the significant harmonics to be included in the quantification of the response. Although the first harmonics (i.e., 1-5 Hz) contain the bulk of the response in this paradigm (i.e., about 86\%, Figure 4), various statistical thresholds have been used for harmonic selection across studies. Adding non-significant harmonics does not cause any harm to the overall response quantification (i.e., if signal = noise, a zero is added to the summed harmonic response) but missing genuine harmonic responses may lead to an underestimated quantification. Hence, a liberal statistical threshold (i.e., $Z>1.64, p<0.05$, one-tailed) is rather recommended at the stage of harmonic selection. Following this procedure, the summed signal amplitude is normalized relative to the surrounding noise through baseline-subtraction. The data can then be averaged within a region-of-interest (ROI). Averaging of the summed signal amplitude across electrodes of a ROI can also be performed before baseline-division (SNR) or a Z-score computation. See Yan et al. (2019) and Dzhelyova et al. (2020) for the most recent analysis procedures applied to the reviewed paradigm.

\subsection{Stimulation parameters}

\subsubsection{Selecting and controlling the stimulus set}

As in many studies, the nature of the face stimulus set can have an important impact on the results and the conclusions reached. The minimum number of individual face stimuli to use in this paradigm is two (AAAABAAAAB...; as in Feuerriegel et al., 2018) but for reasons discussed in the review (Section 3), we advocate using multiple facial identities so that variable individual discriminations are performed at each facial identity change. Ideally, a relatively large set of faces (Figure 2, e.g., at least about 25 faces per gender in most studies) should be used. Since these oddball identities are contrasted against the same base face throughout a stimulation sequence, it is important that the stimulus set is sufficiently homogenous in terms of properties orthogonal to identity, such as gender and ethnicity (i.e., not mixing male and female faces in the same stimulation sequence, or Asian and Caucasian faces, etc.), as well as head orientation, gaze direction, expression, etc. A small set of stimuli carries a risk that the base and oddball faces systematically differ on one of these characteristics (e.g., slight differences in head orientation/gaze direction), thus confounding the FI response not only with low-level image-based factors but also high-level characteristics unrelated to face identity (e.g., Barry-Anwar et al., 2018). 
While the stimulus set should be "naturally homogeneous" (i.e., pictures taken under the same lighting conditions and distance), caution should be taken with extreme homogenization (e.g., by equalizing global luminance, contrast, or spatial frequency content among faces; Rousselet et al., 2008; or presenting all faces in the same oval shape) since it may (1) eliminate genuine visual cues of identity (e.g., differences in shape or surface cues, see Dzhelyova et al., 2014b) and (2) paradoxically introduce confounds by making simple local differences between faces extremely salient. Rather than controlling image differences by elimination/normalization, we advocate a control by variation, i.e., ensuring that the face stimuli vary sufficiently in terms of orthogonal lower-level image cues, such that FI cannot be attributed exclusively one of these cues. For example, faces typically randomly change in size at every stimulus presentation in our studies (minimizing overlap between consecutive images), and a similar manipulation can be applied by additionally randomly varying overall image luminance (Retter et al., in preparation). Another potential manipulation to limit the contribution of image-based cues would be to average the FI response collected in different stimulation sequences performed with different stimulus sets, similarly as to what is sometimes done with sequences of either male or female faces in this paradigm (e.g., Liu-Shuang et al., 2014; Xu et al., 2017).

\subsubsection{Frequency selection}

As discussed above, some stimulation frequencies give rise to larger EEG responses than others not because they "entrain", "resonate with", "borrow" or "build upon" ongoing neural oscillations (Notbohm et al., 2016; Zoefel et al., 2018) but more parsimoniously because they allow to record the highest number of events during a certain amount of time while avoiding interference from overlapping neural responses to these events (Retter \& Rossion, 2016; see Sections 4 \& 6). Nevertheless, stimulation frequency is an important parameter to consider in FPVS paradigms. So what are the reasons why the stimulus presentation frequency $F$ selected in the current FPVS design is often around $6 \mathrm{~Hz}$ (Figure 14), with the frequency of change of facial identity at $F / n$ around 1 $\mathrm{Hz}$ ? 
Originally, $F=6 \mathrm{~Hz}$ was selected not because it gave rise to the largest absolute EEG response with this paradigm, but because it led to the largest facial identity repetition suppression effects (Alonso-Prieto et al., 2013; see also Gentile \& Rossion, 2014 for fMRI evidence; see again Section 5.1.5). In other words, a $6 \mathrm{~Hz}$ stimulus presentation rate optimally enables the measurement of different $v s$. same facial identity processing. It might be thought that setting $F$ to $6 \mathrm{~Hz}$ would prevent measuring harmonic frequency responses of $F / n$ at this rate, such that it would not be ideal in the present oddball paradigm. However, in a more recent study with the current paradigm, we systematically varied $F$ between $40 \mathrm{~Hz}$ and $3 \mathrm{~Hz}$, while keeping the oddball face identity change at $F / n$ constant at $1 \mathrm{~Hz}$ (Figure 20; see Sections $6.2 \&$ 7.5.2; Retter et al., in preparation). Results show that the largest FI response occurred between 4 and $6 \mathrm{~Hz}$. FI responses first emerged at 20 $\mathrm{Hz}$, and their amplitude increased until around $6 \mathrm{~Hz}^{15}$. Moreover, stimulating at $6 \mathrm{~Hz}$ offers a good compromise: at a slower rate, observers would be able to analytically, visually explore the face through eye movements (6 Hz constrains observers to only one fixation for each face); and at a faster rate, observers may not be able to fully extract the facial cues conveying identity information.

The choice of an oddball facial identity change frequency at $F / n=$ around $1 \mathrm{~Hz}$ was made to strike a balance between too short durations separating consecutive oddballs, which could lead to overlapping deflections to identity changes and consequently a reduction of the FI response (see Retter \& Rossion, 2016; and Sections $4.1 \&$ 6.1), and too long inter-stimulus durations, which would reduce the total number of individual face discrimination responses recorded during a stimulation sequence. Somewhat faster $F / n$ frequencies could be used: if FI responses last a little

15 Interestingly, a relatively weaker FI response was found at the slowest $3 \mathrm{~Hz}$ rate. The reasons for this decrease are unclear at the moment: it could be either because at this rate there are only two identical faces in between each change of face identity, reducing RS effects (as addressed in Section 6.2), or because this time duration allows different fixations of the repeated faces, potentially reducing face adaptation. Alternatively, it could be that the FI response is underestimated because the base rate contains more harmonics that are excluded from the FI response quantification (i.e., $3 \mathrm{~Hz}, 6 \mathrm{~Hz}$, etc.). 
over $400 \mathrm{~ms}$ (Section 7.5.2), they could in theory be presented at rates up to $2.5 \mathrm{~Hz}$ without producing overlapping responses. However, an additional aspect to consider with the present paradigm is the ratio of oddball to base faces. For example, when $F=6 \mathrm{~Hz}$ and $F / n=1 \mathrm{~Hz}$, there is a 1/6 oddball presentation ratio, allowing five repetitions of the base face for every oddball face presentation. The relative merit of repetition count $v s$. presentation time of base faces on producing RS effects has not yet been tested empirically with this paradigm. However, at present, we suspect that having at least three base face repetitions is advantageous for measuring FI responses, based on the decreased $F / n$ response at $1 \mathrm{~Hz}$ to a $1 / 3$ oddball incidence when $F$ was varied to $3 \mathrm{~Hz}$ (Retter et al., in preparation; although this proportion of oddball incidence has not yet been tested with other $F$ rates). On these grounds, with a $F$ frequency of $6 \mathrm{~Hz}$, we would recommend having a $F / n$ frequency of less than $2 \mathrm{~Hz}$ (a $1 / 3$ oddball ratio), i.e., $1.5 \mathrm{~Hz}(1 / 4), 1.2 \mathrm{~Hz}(1 / 5)$ or $1 \mathrm{~Hz}(1 / 6)$. Yet, a final aspect to consider is whether there is a sufficiently long SOA to allow for a return to baseline in between oddball face responses, in the case of complementary analyses in the time-domain (see section 7.5 above). For example, if oddball faces are presented at $1.5 \mathrm{~Hz}$, there is an SOA of 667 ms: if the FI response persists for about $500 \mathrm{~ms}$ following stimulus onset, there would be about 167 ms of baseline in between oddball faces. However, if a baseline correction range of one $F$ presentation duration is desired $(167 \mathrm{~ms})$, there is potentially not enough time between oddball faces to apply the correction outside of the FI response. Thus, overall, when $F=6 \mathrm{~Hz}$, we recommend a $F / n$ frequency of either 1.2 or $1 \mathrm{~Hz}$.

In sum, a stimulus presentation frequency of $F=6 \mathrm{~Hz}$ appears to provide a good compromise between enabling strong temporal constraints (i.e., one fixation/face), and generating a maximal FI response, and identity change frequency of $F / n \approx 1 \mathrm{~Hz}$ appears to provide a good compromise between providing enough separation between identity-change responses and enough identity-change events/time, as well a suitable oddball/base ratio. Unfortunately, as discussed above in Section 6.2, efforts are still required to resolve the potential interaction between this $6 \mathrm{~Hz}$ common response and the FI response, in order to properly examine the time-course of this 
function. Moreover, it is an open question whether the optimal frequency range found in Retter et al. (in preparation) stands for other populations than neurotypical adults, and for other stimulus conditions (e.g., different head orientations).

\subsubsection{Stimulation sequence and overall recording duration}

The duration of a stimulation sequence is inversely proportional to the frequency resolution in the frequency-domain (Figure 3). Thus, the longer the stimulation sequences, the higher the frequency resolution of the frequency-domain spectra. While the SNR of the signal amplitude is a function of the number of events presented and the total recording time, a higher frequency resolution can be useful for confining the signal frequency bin to a very limited range, compared to a wide range of local noise exemplars. In the typical paradigm design, sequences are usually about 60 seconds long; however, this duration can be reduced in infant studies (e.g., 20 seconds, de Heering \& Rossion, 2015), or for clinical populations, or for other design constraints (e.g., 7 seconds in a frequency-sweep approach; Retter et al., 2020; Retter et al., in preparation).

In terms of overall recording duration, four minutes (e.g., 4 repetitions of 60 second sequences) were sufficient to provide responses above noise-level (i.e. SNR>1) in $99 \%$ of neurotypical adult individuals and significant responses in $93 \%$ of individuals, with $F=5.88 \mathrm{~Hz}$ and $F / n=1.2 \mathrm{~Hz}$ (Figure 12). Increasing the overall recording duration could nevertheless further increase SNR, but should strike a balance with participant fatigue to avoid being counterproductive.

\subsubsection{Stimulation presentation mode}

Finally, most studies from our laboratory have used a sinewave contrast modulation, which is harmonically simple (at least in the ideal case) and may appear visually comfortable to the participant, but is less adequate for making inferences about the exact response time-courses. A direct comparison between sinewave and squarewave stimulation has not been performed in this paradigm, but is not expected to lead to major differences apart from latency shifts (see Retter \& 
Rossion, 2016; Dzhelyova et al., 2017 for such comparisons with the corresponding face vs. object paradigm or facial expression discrimination, respectively; see also Section 7.5.2).

\subsection{Data analysis and interpretation}

\subsection{1. (Pre)processing and robustness of the data}

The analysis steps for processing this EEG data are fairly straightforward, as described in Figure 21, and a quick look at the data can often be performed without filtering and artifact correction/rejection (see Xu et al., 2017 for data with minimal preprocessing). Of course, the high SNR of the technique does not mean that care should be reduced in collecting high quality EEG data (Luck, 2014). Correction for blinks is not obligatory, and typically only improves the signal for observers who blink above a certain threshold (e.g., 0.2 blinks/second, see Figure 21). Data transformation in SNR is useful for visualizing responses at low (where baseline EEG activity/noise is large; e.g., Figure 4A) and high frequencies (where signal amplitude may be low). A correction by subtraction of the estimated noise, however, has the advantage of keeping the data in microvolt units, which is useful for response quantification across harmonic frequencies (see Appelbaum et al., 2006 for alternative ways of combining the harmonic frequency responses).

Although the oddball FI response can only emerge from a systematic (periodic) amplitude/phase response difference between base and oddball faces (see Sections $4.1 \&$ 6.2), there may be some instances of identity changes that are not detected, when overall a significant oddball response is observed. In principle, periodicity ensures that FI responses are included in the analysis, while the number of FI responses recorded increases the signal sensitivity (similar to eventaveraging in traditional ERP analyses). Thus, missing identity-change event detections may add "blanks" into FI response trial averaging, which reduces the SNR but does not distort response periodicity (see Retter et al., 2020). In support that the FI response is robust against random omissions, it is present exactly at $F / n$ despite observers blinking. The exact relationship between the proportion of identity discriminations and EEG response amplitude with this paradigm (as 
reported for generic face categorization by Retter et al., 2020) would be a useful reference for further inferences about group differences in FI ability (e.g. ASD vs. controls).

\subsubsection{Multi-harmonic response quantification}

A typical response in this paradigm usually spreads over multiple harmonic frequencies, both for the stimulus-presentation rate $F$ (e.g., $6 \mathrm{~Hz}, 12 \mathrm{~Hz}, 18 \mathrm{H}$, etc.) and for the facial identity change rate $F / n$ (e.g., $1.2 \mathrm{~Hz}, 2.4 \mathrm{~Hz}, 3.6 \mathrm{~Hz}$, etc.) (Figure 3; Box 1). In many EEG frequencytagging studies, decisions about consideration of harmonic frequency responses seem arbitrary: often, only the response at the fundamental stimulation frequency, or first harmonic (i.e., $1 F / n$ or $1 F$, is taken into account; but sometimes harmonics of $F / n$ up until $F$ are considered, or only the largest harmonics are selected (see Retter \& Rossion, 2016 for a short discussion of this issue). Although the overall conclusions of these studies may be valid, this procedure risks missing out relevant parts of the response. Indeed, all the harmonic frequencies represent some aspect of the periodic response (Regan, 1989; Norcia et al., 2015). In the oddball FPVS paradigm reviewed here, the FI response clearly spreads over multiple harmonic frequencies, covering a large frequency bandwidth below about $18 \mathrm{~Hz}$, although the bulk of the response is often found below $10 \mathrm{~Hz}$ or even below $6 \mathrm{~Hz}$ (Figure 4). Quantification of the complete FI response requires aggregation of these harmonic frequency responses(see again Figure 3; Box 1; Retter \& Rossion, 2016).

While this spread of the response over multiple harmonic components somewhat complicates quantification, the frequencies containing relevant responses remain completely predictable, as they are by definition integer multiples of the first harmonic/fundamental frequencies. Moreover, such multi-harmonic responses are less distorted by their interference with one another across time, such that more temporal information is preserved: in some cases (very low $F$, or $F / n$ when filtering out $F$ ), such responses can even be defined in terms of their onset latency, as with event-related potentials (see Section 7.5; Retter \& Rossion, 2016). Additionally, the harmonic distribution of the response makes for a richer measure, providing an additional qualitative - characteristics of the response. For example, in the frequency-domain, two individuals 
may have the same overall response amplitude reflecting FI, but their FI response amplitudes may be differentially spread across harmonic frequencies (Figure 3B). This harmonic amplitude distribution is somewhat variable across individuals and relatively stable within individuals, providing an additional marker of the FI function in individuals (Dzhelyova et al., 2019; see Section 7). Another interesting example is provided by developmental studies: as noted above, the bulk of the FI response (about $60 \%$ ) in this paradigm is accounted for by the first harmonic in 5 year old children but only $18 \%$ in adults, providing an additional marker of the developmental trajectory of FI (Lochy et al., 2020; see Section 7.4).

Finally, it is important to stress that a response at $F / n$ is not a "subharmonic" of the stimulus presentation rate, but instead reflects the discrimination between the base and oddball faces at the facial identity change frequency. The notion of subharmonics, which appears in a couple of frequency-tagging papers (most famously, Walter et al., 1946; see also Herrmann, 2001), makes little sense and often merely reflects asymmetries in the onset/offset of the stimulation (see Norcia et al., 2015). Note that in all related paradigms using non-periodic oddball presentations, no subharmonic stimulus-presentation responses have been observed.

\subsubsection{Interpreting FI response amplitude differences}

While the paradigm is applicable as such across all age groups without requiring a high density EEG system thanks to the focal topography of the FI response, the issue of comparing the FI response across age is not negligible. For biophysical reasons, young children tend to have ERP components of particularly high amplitudes, which decrease progressively with age (e.g., Kuefner et al., 2010). This also appears to be the case for the FI response (e.g., close to $1.5 \mu \mathrm{V}$ over occipitotemporal channels in 5 year-olds, Lochy et al., 2020; Figure 18), which is roughly of the same amplitude as in adults. However, their stimulus presentation response is also much larger $(2.1 \mu \mathrm{V}$ in adults, but a response reaching up to $3 \mu \mathrm{V}$ in young children, Figure 5 and Lochy et al., 2020). One option would be therefore to compare the FI response amplitude relative to the stimulus presentation response amplitude, in order to track specific increases of the FI response during 
development. A more promising avenue, however, is the comparison of the percentage of amplitude change for different conditions with the same $F / n$ target frequency, e.g., for upright as compared to inverted faces, or faster $F$ as compared to slower $F$ presentation rates. Initial comparisons across studies reveal important changes between 5 and 8-12 years of age, as discussed in Section 7.4.1 above.

\section{Conclusions and future applications}

The two main goals of this review were to (1) illustrate how to use an oddball FPVS paradigm in EEG to probe the face individuation (FI) function of the human brain as an alternative or as a complement to explicit behavioral tasks and standard ERP measures and (2) summarize findings obtained with this paradigm, supported by a novel large-scale group analysis, in order to reach a better understanding of human FI. Our review of findings reported in 20 published studies and a number of studies in preparation, completed by a large group data analysis, supports the validity, objectivity, sensitivity and reliability of the oddball FPVS EEG FI paradigm. Objectivity and sensitivity are provided by the periodic and fast nature of the stimulation, supporting analyses in the frequency domain. High reliability has been demonstrated empirically, at least for neurotypical adults, even for short experiments, with only a few minutes of recording per condition. As noted in the summary of Section 5 above, validity (i.e., whether FI i truly measured) is supported by a wealth of evidence: substantial reduction by contrast negation and inversion, absence in a well-defined case of prosopagnosia, strong association with functional misperception of identity during intracerebral electrical stimulation, generalization of the response across changes of size, luminance, and head orientation, and the identification of neural sources in the right ventral occipito-temporal cortex with intracerebral EEG. Again, the validity of this measure will have to be continuously evaluated with further studies using the oddball FPVS framework.

The specific neural mechanisms subtending the FI response are thought to essentially reflect suppression of neural activity through the repetition of a single (base) facial identity, contrasting 
with an increase of neural activity to the change of identity (oddballs), at the level of a specific cortical circuit in the (right lateralized) ventral occipito-temporal cortex. Yet, like many EEG signals, the precise neural mechanisms at the cellular level remains unknown and are likely to involve both excitatory and inhibitory cellular responses and interactions in cortical populations of neurons.

Within a few years, the presently reviewed approach has already brought a substantial amount of information regarding the nature of FI (i.e., in terms of facial cues and processes), its task dependency, and even time course. Highly promising data has been collected in neurotypical and atypical populations, across a variety of age groups, showing that the FI function develops progressively and specifically (i.e., independently of general cognitive factors), with a substantial improvement occurring between 5 and 12 years of age. It also appears to be particularly deficient in children with ASD, independently of explicit encoding, maintenance and memory processes for faces.

\subsection{The importance of unfamiliar faces}

We conceptualize FI as a key subfunction of facial identity recognition, the latter consisting of the ability to determine a familiar person's identity from her/his face, together with the specific semantic information or name associated with that person. In order to adequately perform facial identity recognition, one needs to be able to visually individuate faces, i.e., to provide a unique behavioral/neural response to the visual characteristics of each face. Decades-worth of behavioral studies have probed FI with pictures of unfamiliar faces, often devoid of external features, as is also typically the case in the paradigm reviewed here (Figure 2). Yet, some degree of confusion reigns in the field of human face recognition research, because matching such pictures of unfamiliar faces is sometimes seen as unnatural and based on image-based/low-level visual cues (Hancock et al., 2000; Megreya \& Burton, 2006; Jenkins et al., 2011). When more "natural" conditions are used (i.e., unsegmented pictures of faces with different views of the same individual), it is sometimes 
claimed that people are "bad" or "poor" at individuating unfamiliar faces or even that "human expertise (at individuation) is limited to familiar faces" (Young \& Burton, 2018).

However, while it is well established that generalization of identity across views, as well as other face recognition functions (see Ramon \& Gobbini, 2018) can indeed be substantially increased with face familiarity, the processes subtending this improvement remain unclear: while some authors suggest that visual structural encoding of faces is richer for familiar than unfamiliar faces (Bruce et al., 2001; Ramon \& Gobbini, 2018; Young \& Burton, 2018), behavioral improvement at individuating familiar faces could also be essentially or even entirely due to semantic, affective and/or name associations (Dixon et al., 1998; Schwartz \& Yovel, 2016; Rossion, 2018). By measuring FPVS responses implicitly, the paradigm reviewed here could help disentangling these views. Interestingly, a very recent study showed a (modest) increase of the FI oddball response for familiar(ized) over unfamiliar faces, even without requiring generalization across widely different views (Verofsky et al., 2020). However, the individual faces were learned only via semantic associations, and the exact same stimuli were used at learning and in the tested paradigm, limiting clarification of this issue. Future studies could address this issue more systematically, also testing for wider variations of views, expressions, etc. in more natural images, when repeating the same facial identity in the FI oddball paradigm. In principle, more substantial advantages for familiar faces should be recorded in these conditions.

Regardless of the outcome of this issue, the fact that familiar faces are automatically associated with semantic, affective and verbal processes constitute perhaps the very reason why we claim here that unfamiliar faces must generally be preferably used to isolate the visual processes subtending the FI function in the human brain with the present paradigm.

An obvious drawback of measuring FI with unfamiliar faces is that FI could be based to a certain extent on unnatural, analytical processes and low-level visual cues, which, as noted above, may account for above chance performance at explicit behavioral tests in patients with prosopagnosia, infants, or other animal species (including macaque monkeys), especially in the 
context of long or unlimited presentation durations. However, neurotypical human adults appear to have naturally developed a specific level of expertise at unfamiliar FI that is unparalleled in the animal world, based on a specialized network in the right ventral occipito-temporal cortex (Figure 10). Hence, there is no reason to expect that they would predominantly rely on analytical processes or low-level cues to perform unfamiliar FI, even when they have the opportunity to do so (Sergent, 1989). Nevertheless, this is one of the reasons why probing the FI function with unfamiliar faces under the severe constraints of the current oddball FPVS paradigm (i.e., requiring many variable individual face discriminations to be performed at a single glance, automatically, and across substantial size changes) is highly advantageous: it minimizes the contribution of low-level processes and specifically isolates specialized FI, as illustrated by the large effects of inversion, the localization of the response over and inside right occipito-temporal regions, and the absence of a FI response in prosopagnosia. Since it is adept at capturing a high-level FI response, this paradigm is particularly useful for investigating the nature of FI and its neural basis.

\subsection{Functional processes}

Providing that the methodological factors listed above (Section 8) are taken into consideration, the oddball FPVS-EEG paradigm could prove to be extremely useful in future studies for further clarifying the nature of FI. While some stimulus properties have been fine-tuned through empirical testing (e.g., stimulus size change, Dzhelyova et al., 2014), many other parameters warrant systematic investigation. For example, we have mostly used color stimuli in our studies, but how this factor contributes to the FI response is unclear and should be evaluated in future studies. Similarly, we regularly enforce nasion fixation (in line with Peterson \& Eckstein, 2012), but this may not be necessary, or perhaps may even be suboptimal for some individuals (Blais et al., 2008; Peterson \& Eckstein, 2013; Stacchi et al., 2019). As mentioned above, introducing variability to the base and oddball face images, e.g., in terms of viewpoint, would enable testing the extent of generalization of FI for unfamiliar faces (Damon et al., 2020). 
Additionally, further increasing variability of the base facial identity could provide insights as to the impact of low-level adaptation to the base face, and potentially to reduce its impacts on the $F / n$ FI response. Finally, the present paradigm could be straightforwardly combined with "sweep-VEP" stimulation, progressively increasing or decreasing well-defined stimulus properties (e.g., contrast, spatial frequency) while stimulating at fast periodic rates (see Ales et al., 2012; Quek et al., 2018) in order to more specifically characterize the human FI function and enable its comparison across infants, children, other animal species, and machines.

\subsection{Implicit processing, awareness, and periodicity}

The present review has emphasized the lack of explicit task requirement as a virtue of the paradigm, avoiding the contribution of general cognitive and motor processes to the measured response. This does not mean that the non-periodic, orthogonal task typically used (i.e., detection of a change of color or shape of the fixation cross) does not influence the FI response at all. The task, as well as the position of the fixation cross, could be modified or improved, or adapted to cultural and inter-individual differences in optimal fixation position (Blais et al., 2008; Peterson \& Eckstein, 2013) in future studies. For example, according to some authors, familiar faces may be more automatically individuated than unfamiliar faces (Yan et al., 2017; Young \& Burton, 2018). If this is the case, introducing a distracting task, such as detecting stimulus changes orthogonal to identity changes, may potentially reduce the response more for unfamiliar than familiar faces.

Importantly, the fact that the oddball FPVS FI measure is implicit and that participants are not made aware of what is measured does not imply that they do not consciously notice the changes of facial identity during the stimulation sequences. At a $6 \mathrm{~Hz}$ stimulation rate, when probed after the experiment, all participants (except the prosopagnosic patient PS) typically reported that there were frequent changes of facial identity, even though they are unable to tell that these changes were periodic, let alone report the ratio of frequent/deviant responses or the temporal periodicity of the changes of identity (e.g., Liu-Shuang et al., 2016; Retter et al., in preparation). To our knowledge, 
whether participants' knowledge of this ratio/periodicity would modulate the FI response or not has not been tested. Given the fast $6 \mathrm{~Hz}$ stimulation rate typically used, it is difficult to explicitly keep track of image count, and we predict that the FI response would remain unchanged.

In the same vein, an outstanding issue is whether temporal periodicity plays a role in the FI response or not. Studies on the vMMN component with simple stimuli (luminance and orientation changes) indicate that it is abolished or strongly reduced for periodic repetition of oddballs, as used here (Kimura et al. 2010). These observations suggest that periodic and non-periodic temporal changes in facial identity could lead to different responses in the present paradigm. However, given the fast rate of stimulation used here for high level visual discrimination, and in line with the absence of any difference between periodic and non-periodic presentations of natural images of faces in rapid trains of non-face objects (Quek \& Rossion 2017), we think that it is unlikely. A related issue is whether rare "missing oddballs" in a temporally periodic sequence (i.e., AAAABAAAACAAAAA...) would lead to substantial, specific EEG deflections recorded in the frequency- and time-domains, despite the absence of objective FI. Again, in line with the absence of any "missing oddball" effects for faces inserted in rapid trains of non-face objects (Quek \& Rossion 2017) and the results of a variant of the present design with only two facial identity discirminations (Feuerriegel et al., 2018), we think it unlikely. Such studies would nevertheless be important to perform with the present paradigm.

Finally, a related issue is whether the FI responses obtained in the presently reviewed approach are gradual or all-or-none. According to recent evidence also obtained when contrasting faces to objects in FPVS modes, gradually degrading the quality of the stimulus leads to a gradual overall amplitude response decreases (Quek et al., 2018; Retter et al., 2020). However, this effect does not appear to be due to a gradual decrease of individual responses, but rather to fewer all-ornone successful discriminations being performed (Retter et al., 2020). In the case that all-or-none responses are produced, participants' behavioral discrimination accuracy correlates linearly with their neural discrimination response amplitude across conditions, since, e.g., detecting only $80 \%$ 
of changes produces only $80 \%$ of stimulated neural events. Additionally, all-or-none responses predict qualitatively similar responses for degraded and full quality stimuli, e.g., in terms of temporal dynamics and scalp topographies. These indications have been demonstrated for FI with the present oddball FPVS paradigm (Retter et al., in preparation), although explicit examination of recognized $v s$. non-recognized neural FI responses with this paradigm could be tested in future studies. Further clarification on this topic could have implications for our understanding of the nature of the FI function.

\subsection{Inter-individual differences and prosopdysgnosia}

The last two decades have seen an enormous amount of research devoted to study interindividual differences in facial identity recognition, this function being most often probed by explicit behavioral measures of individuation of unfamiliar faces (with various task requirements) (Wilmer, 2017; Stacchi et al., 2020). As noted above in the present review, the sources of this variation remain, however, unknown, and may have little to do with specific visual processes involved in FI. The present approach can help to shed light on this issue, but is also limited by the general neuroanatomical and neurophysiological factors contributing to variations of response amplitude on the scalp. A potential and promising approach to overcome these issues could be to compare responses at $F / n$ across two or more conditions. For example, when various $F$ rates are used, testing for inter-individual differences in resilience to high stimulus presentation rates , using individuals' variable responses at a low frequency rate for normalization (Retter et al., in preparation; see Retter et al., 2020).

The last two decades have also brought a lot of interest in people with lifelong major difficulties in recognizing faces, in the absence of any identified neurological or psychiatric conditions. These individuals are often defined as suffering from developmental prosopagnosia or congenital prosopagnosia (since McConachie, 1976; see e.g., Behrmann \& Avidan, 2005; Duchaine \& Nakayama, 2006; Bate \& Tree, 2017). Despite much research, this condition - which 
should be more accurately referred to as prosopdysgnosia (Rossion, 2018; Sorensen \& Overgaard, 2018) - has been particularly difficult to understand (see e.g., Esins et al., 2016; Geskin \& Berhmann, 2018 and accompanying commentaries). In the absence of clear biological markers, the criteria for defining cases of prosopdysgnosia are variable and disputed, with some individuals even identified based solely on self-report (Kennerknecht et al., 2006; see Barton \& Corrow, 2016). Since standard ERP components, such as the N170 and N250r, have been reported to be abnormally reduced at the group level in prosopdysgnosia (Towler et al., 2012; Parkteny et al., 2015; Fisher et al., 2017), there is hope that the FI index described here could serve as an objective, sensitive, and reliable biomarker of the condition. In fact, several research groups are currently working to compare the $F / n$ FI measures in independently selected prosopdysgnosic individuals and controls. A key issue, however, is whether prosopdysgnosia exists as a (specific) disorder, or whether it merely reflects the lower tail of the normal population's distribution of face recognition abilities (Barton \& Corrow, 2016). In the latter case, we will revert to the issue of inter-individual differences in FI, as discussed above.

\subsection{Typical and atypical development}

Finally, as already illustrated in the present review, this approach may be extremely valuable in the future to track the typical neurodevelopment of the FI function, which remains highly controversial (McKone et al., 2012). Although infants may not be able to individuate pictures of unfamiliar faces as typically used in this paradigm (see Section 7.4), an interesting issue would be to test infants with the present paradigm, except comparing pictures of unfamiliar and highly familiar faces, either directly in the same stimulation sequence (e.g., the primary caregiver's face as a base face, with unfamiliar faces as oddballs) or indirectly (i.e., stimulation sequences with familiar(ized) faces only compared to sequences with unfamiliar faces; see Verofsky et al., 2020 in adults). It may well be that when their use of low-level cues is limited in a FPVS paradigm, infants' FI abilities are limited to a few highly familiar faces. 
Studies of atypical neurodevelopment as in ASD boys with this paradigm (Vettori et al., 2019b) are also highly promising, not only thanks to the clarity and the specificity of the FI response differences at the group level, but also due to the high discriminability between individual ASD children and controls, within only a few minutes of recording. This approach may therefore open an avenue for developing a reliable biomarker of (social) developmental disorders affecting FI perception, such as ASD, in young children or even infants (Rossion, 2020). In addition, it could be combined with other FPVS EEG paradigms, e.g., measuring facial emotion discrimination (Dzhelyova et al., 2017), in order to provide more specific indications.

Moreover, based on performance at explicit behavioral tasks, such as the CFMT or GFMT, it has been claimed claim that FI peaks at about 30 years of age (Germine et al., 2011) and decreases in elderly people (Megreya \& Bindemann, 2015). However, as discussed early in this review, there is no evidence that this developmental function truly reflects FI rather than general processes involved in these tasks, so that this issue would also benefit from in-depth investigation with the present oddball FPVS paradigm. Still, at the other end of development, oddball FPVS measures of FI, which appear to be based on repetition suppression effects (i.e., the simplest form of neural plasticity) could also be used to probe early difficulties in memory encoding as in neurodegenerative disorders, such as Alzheimer's disease. 


\section{Supplementary Material: Methods of the large-scale group analysis}

\section{Data sample}

The dataset for the large group analysis was sampled from 7 different studies from within our laboratory (Dzhelyova et al., 2014a; Dzhelyova et al., 2019; Hagen, Laguesse et al., in preparation; Or, Liu-Shuang et al., in preparation; as well as unpublished data: please see Acknowledgements). We included all the available data of the oddball fast periodic visual stimulation paradigm with faces shown at $\sim 6 \mathrm{~Hz}$, face identity changes occurring either at $1 / 5(\sim 1.2$ $\mathrm{Hz})$ or $1 / 7(\sim 0.86 \mathrm{~Hz})$ intervals, and EEG acquired with the same 128-channel system. All data were acquired with a Biosemi ActiveTwo system, sampled at $512 \mathrm{~Hz}$. It is available as epoched EEG signals (i.e., pre-FFT) in a Letswave format here upon publication of the paper: http://doi.org/10.4121/uuid:8e8cfaf2-ab00-45b2-90a0-623fabf75ca9.

These studies were collected from a total of 152 neurotypical adult participants, some of whom were tested in multiple studies. All the participants gave written informed consent in the accordance with the guidelines of the Bioethical Committee of the University of Louvain and were monetarily compensated for their time. All of them have also completed a computerized version of the Benton Face Matching Test prior to EEG testing. A final data sample from 130 unique participants was selected $(79$ females, mean age $=21.58 \pm 2.21)$ based on Benton scores $>40$, and correct EEG data quality. A subset of 79 participants (48 females) also completed the task for both upright and inverted faces.

\section{Frequency-domain analysis}

The selected datasets underwent various different preprocessing steps, including bandpass filtering, downsampling, and artefact reject. As the last step, all the data were re-referenced to the average of all EEG channels. The current group-level frequency-domain analysis began with cropping the preprocessed data into $60 \mathrm{~s}$ epochs containing an integer number of the respective oddball frequency presentation durations ( 200-280). The segmented time-domain epochs were 
then transformed into the frequency-domain in two ways: 1) either by first averaging the epochs before applying a FFT, or 2) the inverse. The number of epochs averaged before FFT was also varied in order to examine the effects of stimulation duration on the signal-to-noise ratio (SNR) of the face individuation response.

\section{Quantification of the (oddball) face individuation response.}

We extracted the amplitudes at oddball harmonic frequency $F / n$ and its harmonics up to 20 $\mathrm{Hz}$ (excluding stimulus presentation frequency harmonics), as well as the 22 surrounding frequency bins representing background EEG noise. These amplitude "chunks" were summed and then baseline-corrected by subtracting from the summed oddball amplitude the mean noise amplitude from the 10 neighboring bins, excluding the immediately adjacent bins (see Figure 21). Unless otherwise specified, response amplitude of the face individuation response refers to these corrected and summed amplitudes. Response significance was assessed in a similar way: after summing the chunk amplitudes, a Z-score transform was computed with the 10 neighboring bins (mean noise subtracted from the oddball amplitude and divided by the standard deviation of the noise). Based on the topographical analysis of the face individuation response, we focused further analysis on a bilateral occipito-temporal (OT) region-of-interest (ROI) composed of 10 channels (P8, P10, PO8, PO10, PO12, P7, P9, PO7, PO9, PO11; see Figure 6A).

\section{Quantification of the general visual response}

The quantification of the general visual response at the stimulus presentation frequency $F$ and its harmonics was similar to the above procedure for the face individuation response, except that harmonics were summed up to $50 \mathrm{~Hz}$. The ROI was also slightly different, containing 10 electrodes over the medial occipital and right parietal/occipito-temporal regions (Oz, Oiz, Iz, POOz, O2, O1, POO6, PO8, POI2, PO10; see Figure 14C). 


\section{Time-domain analysis}

Only data with a downsampled rate of $256 \mathrm{~Hz}$ were retained for the time-domain analysis (5/7 studies, $\mathrm{N}=110$ in the upright condition). We first applied a $4^{\text {th }}$ order Butterworth low-pass filter with cut-off at $30 \mathrm{~Hz}$ and then segmented the data to contain an integer number of oddball presentation durations, as described above. A multi-notch filter centered on the stimulus presentation frequency was applied to selectively eliminate the general visual responses up to 48 $\mathrm{Hz}$ (FFT filter, width $=0.02 \mathrm{~Hz}$ ). We next extracted $1000 \mathrm{~ms}$ epochs time-locked to the onset of the oddball facial identity changes, containing a baseline lasting one stimulation duration (167 ms or $170 \mathrm{~ms})$. We further discarded noisy epochs containing deflections exceeding $100 \mu \mathrm{V}$ for each subject and condition, and kept only files with fewer than $10 \%$ of rejected epochs. The remaining epochs $(\mathrm{N}=80)$ were averaged and baseline-corrected relative to the pre-stimulus baseline (either $167 \mathrm{~ms}$ or $170 \mathrm{~ms}$ ). For the comparison of upright vs. inverted faces, we only considered files meeting the artifact rejection criterion in both upright and inverted conditions, resulting in a final sample of 44 subjects. 


\section{Acknowledgments}

Unpublished data for the large-scale group analysis: Katrien Torfs, Charles Or, Milena Dzhelyova, and authors Joan Liu-Shuang and Bruno Rossion. The authors thank Meike Ramon, Sofie Vettori, Angélique Volfart, Simen Hagen, and two anonymous reviewers for their helpful comments on a previous version of this manuscript. 


\section{References}

Abudarham, N. \& Yovel, G. (2020). Face recognition depends on specialized mechanisms tuned to view-invariant facial features: Insights from deep neural networks optimized for face or object recognition. https://www.biorxiv.org/content/10.1101/2020.01.01.890277v1.

Adibpour, P., Dubois, J., \& Dehaene-Lambertz, G. (2018). Right but not left hemispheric discrimination of faces in infancy. Nature Human Behaviour, 2, 67-79.

Adrian, E. D. (1944). Brain rhythms. Nature, 153, 360-362.

Adrian, E. D., \& Matthews, B. H. C. (1934). The interpretation of potential waves in the cortex. The Journal of Physiology, 81, 440-471.

Ales, J., Farzin, F., Rossion, B., Norcia, A.M. (2012). An objective method for measuring face detection thresholds using the sweep steady-state evoked response. Journal of Vision, 12:18, $1-18$.

Alonso-Prieto, E.A., Van Belle, G., Liu, J., Norcia, A.M., \& Rossion, B. (2013). The 6 Hz fundamental stimulation frequency rate for individual face discrimination in the right occipitotemporal cortex. Neuropsychologia, 51, 2863-2975.

Ambrus, G.G., Windel, F., Burton, A.M., \& Kovács G. (2017). Causal evidence of the involvement of the right occipital face area in face-identity acquisition. Neuroimage, 148, 212-218

Amihai, I., Deouell, L. Y., \& Bentin, S. (2011). Neural adaptation is related to face repetition irrespective of identity: a reappraisal of the N170 effect. Experimental Brain Research, 209, 193-204.

Anzellotti, S., Fairhall, S. L., \& Caramazza, A. (2014). Decoding representations of face identity that are tolerant to rotation. Cerebral Cortex, 24, 1988-1995.

Appelbaum, L.G., Wade, A.R., Vildavski, V.Y., Pettet, M.W., Norcia, A.M. (2006) Cue-invariant networks for figure and background processing in human visual cortex. $J$ Neurosci., 26, 11695-11708.

Axelrod, V. \& Yovel, G. (2013). The challenge of localizing the anterior temporal face area: a possible solution. NeuroImage, 8, 371-80.

Bach, M., \& Meigen, T. (1999). Do's and don'ts in Fourier analysis of steady-state potentials. Doc. Ophthalmol., 99, 69-82.

Barrett, S.E., Rugg, M.D., \& Perrett, D.I. (1988). Event-related potentials and the matching of familiar and unfamiliar faces. Neuropsychologia, 26, 105-17.

Barry-Anwar, R., Hadley, H., Conte, S., Keil, A., \& Scott, L. S. (2018). The developmental time 
course and topographic distribution of individual-level monkey face discrimination in the infant brain. Neuropsychologia, 108, 25-31.

Barton, J.J. (2008). Structure and function in acquired prosopagnosia: lessons from a series of 10 patients with brain damage. J Neuropsychol., 2, 197-225.

Barton, J.J., \& Corrow, S.L. (2016). Selectivity in acquired prosopagnosia: The segregation of divergent and convergent operations. Neuropsychologia, 83, 76-87.

Bate S., \& Tree J.J. (2017). The definition and diagnosis of developmental prosopagnosia. QJ Exp Psychol. 70, 193-200.

Behrmann, M, \& Plaut, D.C. (2013) Distributed circuits, not circumscribed centers, mediate visual recognition. Trends in Cognitive Sciences, 17, 210-219.

Behrmann, M, Thomas, C., \& Humphreys, K. (2006). Seeing it differently: visual processing in autism. Trends in Cognitive Sciences, (10.6), 258-264.

Behrmann, M., \& Avidan, G. (2005). Congenital prosopagnosia: face-blind from birth. Trends Cogn Sci., 9, 180-187.

Behrmann, M., Avidan, G., Leonard, G. L., Kimchi, R., Luna, B., Humphreys, K., \& Minshew, N. (2006). Configural processing in autism and its relationship to face processing. Neuropsychologia, 44(1), 110-129.

Bekhtereva, V., \& Müller, M.M. (2015). Affective facilitation of early visual cortex during rapid picture presentation at 6 and 15 Hz. Soc Cogn Affect Neurosci. 10, 1623-1633.

Bekhtereva, V., Pritschmann, R., Keil, A., \& Müller, M.M. (2018). The neural signature of extracting emotional content from rapid visual streams at multiple presentation rates: A crosslaboratory study. Psychophysiology, 55(12):e13222.

Bentin, S., McCarthy, G., Perez, E., Puce, A., \& Allison, T. (1996). Electrophysiological studies of face perception in humans. Journal of Cognitive Neuroscience, 8, 551-565.

Benton, A. (1990). Facial Recognition 1990. Cortex, 26, 491-499.

Benton, A. L., \& Van Allen, M. W. (1968). Impairment in facial recognition in patients with cerebral disease. Transactions of the American Neurological Association, 93, 38-42.

Benton, A.L., \& Van Allen, M.W. (1972). Prosopagnosia and facial discrimination. Journal of Neurological Sciences, 15, 167-172.

Berger, H. (1929). Uber das Elektroenkephalogramm des Menschen, Arch. Psychiat. Nervenkr. 87 , $527-570$.

Bindemann, M., Avetisyan, M., \& Rakow, T. (2012). Who can recognize unfamiliar faces? Individual differences and observer consistency in person identification. $J$ Exp Psychol Appl. 18, 277-291. 
Bindemann, M., Fysh, M., Cross, K., \& Watts, R. (2016). Matching faces against the clock. $i$ Perception, 7(5), 2041669516672219.

Blais, C., Jack, R.E., Scheepers, C., Fiset, D., Caldara, R. (2008). Culture Shapes How We Look at Faces. PLoS One, 3(8):e3022.

Bodamer, J. (1947). Die-Prosop-agnosie. Archives Psychiatrie Nervenkrankh, 179, 6-54.

Bottari, D., Dormal, G., Bednaya, E., Villwock, A., Dzhelyova, M., Grin, K., Pietrin, P., Ricciardi, E., Rossion, B. \& Röder, B. (submitted). EEG frequency-tagging demonstrates differential neural face processing in congenitally deaf signers.

Bouvier, S. E., \& Engel, S. A. (2006). Behavioral deficits and cortical damage loci in cerebral achromatopsia. Cerebral Cortex, 16, 183-191.

Bowles, D.C., McKone, E., Dawel, A., Duchaine, B., Palermo, R., Schmalzl, L., Rivolta, D., Wilson, E., \& Yovel, G. (2009). Diagnosing prosopagnosia: Effects of ageing, sex, and participant-stimulus ethnic match on the Cambridge Face Memory Test and Cambridge Face Perception Test. Cognitive Neuropsychology, 26, 423-455.

Braddick, O. J., Wattam-Bell, J., \& Atkinson, J. (1986). Orientation-specific cortical responses develop in early infancy. Nature, 320, 617-619.

Braddick, O., Birtles, D., Wattam-Bell, J., \& Atkinson, J. (2005). Motion- and orientation- specific cortical responses in infancy. Vision Research, 45, 3169-3179.

Brecht, K.F., Wagener, L., Ostojić, L., Clayton, N.S. \& Nieder, A. (2017). Comparing the face inversion effect in crows and humans. Journal of Comparative Physiology A, 203, 1017-1027.

Bruce ,V. (1982). Changing faces: visual and non-visual coding processes in face recognition. $\mathrm{Br}$ J Psychol. 73, 105-116.

Bruce, V., \& Langton, S. R. H. (1994). The use of pigmentation and shading information in recognising the sex and identities of faces. Perception, 23, 803-822.

Bruce, V., \& Young, A. (1998). In the eye of the beholder: The science of face perception. New York: Oxford University Press.

Bruce, V., Henderson, Z., Greenwood, K., Hancock, P.J.B., Burton, A. M., \& Miller, P. (1999). Verification of face identities from images captured on video. Journal of Experimental Psychology: Applied, 5, 339-360.

Bruce, V., Henderson, Z., Newman, C., \& Burton, A.M. (2001). Matching identities of familiar and unfamiliar faces caught on CCTV images. J Exp Psychol Appl., 7207-18

Bruyer, R. \& Brysbaert, M., (2011). Combining Speed and Accuracy in Cognitive Psychology: Is the Inverse Efficiency Score (IES) a Better Dependent Variable than the Mean Reaction Time (RT) and the Percentage Of Errors (PE)? Psychologica Belgica, 51, 5-13 
Burton, A.M., White, D., \& McNeill, A. (2010). The Glasgow Face Matching Test. Behavior Research Methods, 42, 286-291.

Bushnell, I. W. R., Sai, F., \& Mullin, J. T. (1989). Neonatal recognition of the mother's face. British journal of Developmental Psychology, 7, 3-15.

Busigny, T., Graf, M., Mayer, E., Rossion, B. (2010). Acquired prosopagnosia as a face-specific disorder: Ruling out the general visual similarity account . Neuropsychologia, 48, 2051-2067.

Caharel, S., d'Arripe, O., Ramon, M., Jacques, C., \& Rossion, B. (2009). Early adaptation to unfamiliar faces across viewpoint changes in the right hemisphere: evidence from the N170 ERP component. Neuropsychologia, 47, 639-643.

Campatelli, G., Federico, R. R., Apicella, F., Sicca, F., \& Muratori, F. (2013). Face processing in children with ASD: Literature review. Research in Autism Spectrum Disorders, 7, 444-454.

Capilla, A., Pazo-Alvarez, P., Darriba, A., Campo, P., \& Gross, J. (2011). Steady-state visual evoked potentials can be explained by temporal superposition of transient event-related responses. PLoS One, 6(1), e14543.

Carandini, M., Heeger, D.J., 2011. Normalization as a canonical neural computation. Nat Rev Neurosci, 13, 51-62.

Carey, S. (1981). The development of face perception. In G. Davies \& H. D. Ellis (Eds.), Perceiving and remembering faces. London: Academic Press.

Carey, S. (1992). Becoming a face expert. Philosophical Transactions of the Royal Society of London. Series B, Biological Sciences, 335, 93-95.

Carey, S., \& Diamond, R. (1977). From piecemeal to configurational representation of faces. Science. 195, 312-314

Carlson, T., Tovar, D.A., Alink, A., Kriegeskorte, N. (2013). Representational dynamics of object vision: the first $1000 \mathrm{~ms}$. Journal of Vision, 13(10).

Chawarska, K., Macari, S., \& Shic, F. (2013). Decreased spontaneous attention to social scenes in 6-month-old infants later diagnosed with autism spectrum disorders. Biological Psychiatry, 74(3), 195-203.

Chung J., Lee J., Park H.J., Yang H.S. (2006). Viewpoint-Invariant Face Recognition Based on View-Based Representation. In: Huang DS., Li K., Irwin G.W. (eds) Computational Intelligence. ICIC 2006. Lecture Notes in Computer Science, vol 4114. Springer, Berlin, Heidelberg.

Cichy, R.M., Pantazis, D., Oliva, A. (2014). Resolving human object recognition in space and time. Nat. Neurosci., 17, 455-462.

Collins, E., Robinson, A.K., \& Behrmann, M. (2018). Distinct neural processes for the perception 
of familiar versus unfamiliar faces along the visual hierarchy revealed by EEG. Neuroimage, $181,120-131$.

Collishaw, S.M., \& Hole, G.J. (2000). Featural and configurational processes in the recognition of faces of different familiarity. Perception, 29, 893-909.

Courchesne, E., Ganz, L. \& Norcia, A.M. (1981). Event-Related Brain Potentials to Human Faces in Infants. Child Development, 52, 804-811.

Crookes K, McKone E. (2009). Early maturity of face recognition: no childhood development of holistic processing, novel face encoding, or face-space. Cognition, 111(2):219-47.

Crookes, K., \& McKone, E. (2009). Early maturity of face recognition: No childhood development of holistic processing, novel face encoding, or face-space. Cognition, 111, 219-247.

Croydon A, Pimperton H, Ewing L, Duchaine B.C., Pellicano E. (2014). The Cambridge Face Memory Test for Children (CFMT-C): a new tool for measuring face recognition skills in childhood. Neuropsychologia, 62, 60-67.

Damon, F., Leleu, A., Rekow, D., Poncet, F., \& Baudouin, J.Y. (2020). Expertise for conspecific face individuation in the human brain. Neuroimage. 204:116218.

Davidesco, I., Zion-Golumbic, E., Bickel, S., Harel, M., Groppe, D.M., Keller, C.J., Schevon, C.A., McKhann, G.M., Goodman, R.R., Goelman, G., Schroeder, C.E., Mehta, A.D, \& Malach, R. (2014). Exemplar selectivity reflects perceptual similarities in the human fusiform cortex. Cerebral Cortex. 24, 1879-1893.

Davidoff, J., \& Landis, T. (1990). Recognition of unfamiliar faces in prosopagnosia. Neuropsychologia, 28, 1143-1161.

Davies-Thompson, J., Gouws, A., \& Andrews, T. J. (2009). An image-dependent representation of familiar and unfamiliar faces in the human ventral stream. Neuropsychologia, 47, 1627-1635.

Dawson, G. D. (1954). A summation technique for the detection of small evoked potentials. Electroencephalogr Clin Neurophysiol, 6, 65-84.

Dawson, G., Carver, L., Meltzoff, A. N., Panagiotides, H., McPartland, J., \& Webb, S. J. (2002). Neural correlates of face and object recognition in young children with autism spectrum disorder, developmental delay, and typical development. Child Development, 73, 700-717.

Dawson, G., Webb, S. J., \& McPartland, J. (2005). Understanding the Nature of Face Processing Impairment in Autism: Insights From Behavioral and Electrophysiological Studies. Dev Neuropsychol. 27, 403-424.

de Haan, M., Pascalis, O., \& Johnson, M. H. (2002). Specialization of neural mechanisms underlying face recognition in human infants. Journal of Cognitive Neuroscience, 14, 199209. 
de Heering, A., \& Rossion, B. (2015). Rapid categorization of natural face images in the infant right hemisphere. ELife, 4, 1-14.

de Heering, A., Rossion, B., Maurer, D. (2012). Developmental Changes in Face Recognition During Childhood: Evidence from Upright and Inverted Faces. Cognitive Development, 27 , $17-27$.

De Renzi, E, \& Spinnler, H. (1966). Facial recognition in brain-damaged patients. An experimental approach. Neurology, 16, 145-52.

Dehaene, S., Cohen, L., Morais, J., \& Kolinsky, R. (2015). Illiterate to literate : behavioural and cerebral changes induced by reading acquisition. Nature, 16(4), 234-244.

Delvenne, J. F., Seron, X., Coyette, F., \& Rossion, B. (2004). Evidence for perceptual deficits in associative visual (prosop)agnosia: a single-case study. Neuropsychologia, 42(5), 597-612.

Dennett, H.W., McKone, E., Tavashmi, R., Hall, A., Pidcock, M., Edwards, M., \& Duchaine B. (2012). The Cambridge Car Memory Test: a task matched in format to the Cambridge Face Memory Test, with norms, reliability, sex differences, dissociations from face memory, and expertise effects. Behav Res Methods, 44(2):587-605.

Di Carlo, J.J., \& Cox, D.D. (2007). Untangling invariant object recognition. Trends Cogn Sci. 11(8):333-41.

Dixon, M.J., Bub, D.N., \& Arguin, M. (2008). Semantic and visual determinants of face recognition in a prosopagnosic patient. J Cogn Neurosci., 10, 362-376.

Duchaine, B., \& Yovel, G. (2015). A revised neural framework for face processing. Annual Review of Vision Science, 1, 393-416.

Duchaine, B.C., \& Nakayama, K. (2006). The Cambridge face memory test: Results for neurologically intact individuals and and an investigation of its validity using inverted face stimuli and prosopagnosic participants. Neuropsychologia, 44, 576-585.

Duchaine, B.C., Germine, L., \& Nakayama, K. (2007). Family resemblance: Ten family members with prosopagnosia and within-class object agnosia. Cognitive Neuropsychology, 24, 419430.

Dwyer, P., Xu, B., \& Tanaka, J. W. (2018). Investigating the perception of face identity in adults on the autism spectrum using behavioural and electrophysiological measures. Vision Research, 157, 132-141.

Dyer, A. G., Neumeyer, C., \& Chittka, L. (2005). Honeybee (Apis mellifera) vision can discriminate between and recognise images of human faces. The Journal of Experimental Biology, 208(Pt 24), 4709-4714.

Dzhelyova M, Rossion B. (2014). Supra-additive contribution of shape and surface information to 
individual face discrimination as revealed by fast periodic visual stimulation. Journal of Vision, 14(14):15, 1-14.

Dzhelyova M, Rossion B. (2014). The effect of parametric stimulus size variation on individual face discrimination indexed by fast periodic visual stimulation. BMC Neurosci. 15(1):87.

Dzhelyova, M. Schiltz, C., \& Rossion, B. (in press). The relationship between the benton face recognition test and electrophysiological unfamiliar face individuation response as revealed by fast periodic visual Stimulation. I-Perception.

Dzhelyova, M., Jacques, C., Dormal, G., Michel, C., Schiltz, C., \& Rossion, B. (in press). High test-retest reliability of a neural index of rapid automatic discrimination of unfamiliar individual faces. Visual Cognition, 27, 127-141.

Dzhelyova, M., Jacques, C., Rossion, B. (2017). At a single glance: fast periodic visual stimulation uncovers the spatio-temporal dynamics of brief facial expression changes in the human brain. Cerebral Cortex, 8, 4106-4123.

Edelman, G.M. (1993). Neural Darwinism: selection and reentrant signaling in higher brain function. Neuron, 10, 115-125.

Eimer, M, Kiss, M, \& Nicholas, S. (2010). Response profile of the face-sensitive N170 component: a rapid adaptation study. Cerebral Cortex. 20, 2442-2452.

Ellis, H. D. (1975). Recognising faces. British Journal of Psychology, 66, 409-426.

Emery, N.J., Lorincz, E.N., Perrett, D.I., Oram, M.W., and Baker, C.I. (1997). Gaze following and joint attention in rhesus monkeys (Macaca mulatta). J Comp Psychol. 111, 286-93.

Engell, A. D., \& McCarthy, G. (2010). The relationship of gamma oscillations and face-specific ERPs recorded subdurally from occipitotemporal cortex. Cerebral Cortex, 21(5), 1213-1221.

Engst, F. M., Martín-Loeches, M., \& Sommer, W. (2006). Memory systems for structural and semantic knowledge of faces and buildings. Brain Research, 1124, 70-80.

Esins J, Schultz J, Stemper C, Kennerknecht I, Bülthoff I. (2016). Face Perception and Test Reliabilities in Congenital Prosopagnosia in Seven Tests. Iperception, 7(1):2041669515625797

Estudillo, A.J., \& Bindemann, M. (2014). Generalization across view in face memory and face matching. Iperception, 7, 589-601

Ewbank, M. P., Henson, R. N., Rowe, J. B., Stoyanova, R. S., \& Calder, A. J. (2013). Different neural mechanisms within occipitotemporal cortex underlie repetition suppression across same and different-size faces. Cerebral Cortex, 23(5), 1073-1084.

Farah, M. J. (1990). Visual agnosia: Disorders of object recognition and what they tell us about normal vision. Cambridge, MA, US: The MIT Press. 
Feuerriegel, D., Keage, H., Rossion, B., Quek, G. (2018). Immediate stimulus repetition abolishes stimulus expectation and surprise effects in fast periodic visual oddball designs. Biological Psychology, 138, 110-125.

Fisher, K., Towler, J., \& Eimer, M. (2017). Face identity matching is selectively impaired in developmental prosopagnosia. Cortex. 89, 11-27

Freire, A., Lee, K., \& Symons, L. A. (2000). The face-inversion effect as a deficit in the encoding of configural information: Direct evidence. Perception, 29, 159-170.

Freiwald, W.A., \& Tsao, D.Y. (2010). Functional compartmentalization and viewpoint generalization within the macaque face-processing system. Science. 330(6005):845-851.

Friston, K., (2005). A theory of cortical responses. Philos. Trans. R. Soc. Lond. B: Biol. Sci. 360 (1456), 815-836.

Fysh, M. C., \& Bindemann, M. (2017). Effects of time pressure and time passage on face-matching accuracy. Royal Society open science, 4(6), 170249.

Fysh, M. C., \& Bindemann, M. (2018). The Kent face matching test. British Journal of Psychology, 109(2), 219-231.Galambos, R., Makeig, S., \& Talmachoff, P. J. (1981). A 40-Hz auditory potential recorded from the human scalp. Proceedings of the National Academy of Sciences of the United States of America, 78, 2643-2647.

Galambos, R., Makeig, S., \& Talmachoff, P.J. (1981). A 40-Hz auditory potential recorded from the human scalp. Proc Natl Acad Sci U S A, 78, 2643-2647

Galper, R. E. (1970). Recognition of faces in photographic negative. Psychonomic Science, 19, 207-208.

Gao, X., Gentile, F., Rossion, B. (2018). Fast Periodic Stimulation (FPS): A highly effective approach in fMRI brain mapping. Brain Structure and Function, 223, 2433-2454.

Gao, X., Vuong, Q.C., \& Rossion, B. (2019). The cortical face network of the prosopagnosic patient PS with fast periodic stimulation in fMRI. Cortex, 119, 528-542.

Gaspar, C.M., Rousselet, G.A., \& Pernet, C.R. (2011). Reliability of ERP and single-trial analyses. Neuroimage, 58, 620-629.

Gauthier, I., Tarr, M. J., Moylan, J., Skudlarski, P., Gore, J. C., \& Anderson, a W. (2000). The fusiform "face area" is part of a network that processes faces at the individual level. Journal of Cognitive Neuroscience, 12, 495-504.

Gentile, F., Rossion, B. (2014). Temporal frequency tuning of cortical face-sensitive areas for individual face perception. Neuroimage, 90, 256-265.

Germine L, Nakayama K, Duchaine BC, Chabris CF, Chatterjee G, Wilmer JB. (2012). Is the Web as good as the lab? Comparable performance from Web and lab in cognitive/perceptual 
experiments. Psychon Bull Rev., 19, 847-57

Germine LT, Duchaine B, Nakayama K. (2011). Where cognitive development and aging meet: face learning ability peaks after age 30. Cognition. 118, 201-10.

Geskin, J., \& Behrmann, M. (2018). Congenital prosopagnosia without object agnosia? A literature review. Cogn Neuropsychol. 35, 4-54.

Gignac, G.E., Shankaralingam, M., Walker, K., Kilpatrick, P. (2016). Short-term memory for faces relates to general intelligence moderately. Intelligence, 57, 96-104.

Gilaie-Dotan, S., Gelbard-Sagiv, H., \& Malach, R. (2010). Perceptual shape sensitivity to upright and inverted faces is reflected in neuronal adaptation. NeuroImage, 50, 383-395.

Goesaert, E., \& Op de Beeck, H.P. (2013). Representations of facial identity information in the ventral visual stream investigated with multivoxel pattern analyses. J Neurosci., 33, 8549-5

Gohel, B., Lim, S., Kim, M.Y., Kwon, H., \& Kim K. (2018). Dynamic pattern decoding of sourcereconstructed MEG or EEG data: Perspective of multivariate pattern analysis and signal leakage. Comput Biol Med., 93, 106-116.

Golarai, G., Ghahremani, D. G., Whitfield-Gabrieli, S., Reiss, A., Eberhardt, J. L., Gabrieli, J. D. E., \& Grill-Spector, K. (2007). Differential development of high-level visual cortex correlates with category-specific recognition memory. Nature Neuroscience, 10(4), 512-522.

Golarai, G., Liberman, A., \& Grill-Spector, K. (2017). Experience Shapes the Development of Neural Substrates of Face Processing in Human Ventral Temporal Cortex. Cereb Cortex, 27, 1229-1244.

Gregory, R. (1980). Perceptions as hypotheses. Philosophical Transactions of the Royal Society of London. Series B, Biological Sciences, 290, 181-197.

Griffin, J.W. (2020). Quantifying the face inversion effect in nonhuman primates: a phylogenetic meta-analysis. Animal Cognition, 23, 237-249.

Grill-Spector, K., \& Malach, R. (2001). FMR-adaptation: A tool for studying the functional properties of human cortical neurons. Acta Psychologica, 107, 293-321.

Grill-Spector, K., Henson, R., \& Martin, A. (2006). Repetition and the brain: neural models of stimulus-specific effects. Trends in Cognitive Sciences, 10, 14-23.

Grill-Spector, K., Kushnir, T., Edelman, S., Avidan, G., Itzchak, Y., and Malach, R. (1999). Differential processing of objects under various viewing conditions in the human lateral occipital complex. Neuron, 24, 187-203.

Grill-Spector, K., Weiner, K. S., Kay, K. \& Gomez, J. (2017). The functional neuroanatomy of human face perception. Annual Review of Vision Science, 3, 167-196.

Grootswagers T, Wardle SG, Carlson TA. (2017). Decoding Dynamic Brain Patterns from Evoked 
Responses: A Tutorial on Multivariate Pattern Analysis Applied to Time Series Neuroimaging Data. J Cogn Neurosci. 29, 677-697.

Grotheer, M., Kovács, G., (2015). The relationship between stimulus repetitions and fulfilled expectations. Neuropsychologia, 67, 175-182

Gruss, L. F., Wieser, M. J., Schweinberger, S. R., \& Keil, A. (2012). Face-evoked steady-state visual potentials: effects of presentation rate and face inversion. Front. Hum. Neurosci., 6, 316.

Guntupalli, J. S., Wheeler, K. G., \& Gobbini, M. I. (2017). Disentangling the Representation of Identity from Head View Along the Human Face Processing Pathway. Cerebral Cortex, 27, $46-53$.

Hagen S, Tanaka JW. (2019). Examining the neural correlates of within-category discrimination in face and non-face expert recognition. Neuropsychologia. 124, 44-54.

Halit, H., de Haan, M., \& Johnson, M.H. (2003). Cortical specialisation for face processing: facesensitive event-related potential components in 3- and 12-month-old infants. Neuroimage. 19, 1180-1193.

Hamsher, K.D., Levin, H.S., \& Benton A.L. (1979). Facial recognition in patients with focal brain lesions. Arch Neurol. 36, 837-9.

Hancock, P.J., Bruce, V.V., \& Burton, A.M. (2000). Recognition of unfamiliar faces. Trends Cogn Sci., 4, 330-337.

Haxby, J. V, Ungerleider, L. G., Clark, V. P., Schouten, J. L., Hoffman, E. a, \& Martin, A. (1999). The effect of face inversion on activity in human neural systems for face and object perception. Neuron, 22(1), 189-199.

Haxby, J. V., Hoffman, E. A., \& Gobbini, M. I. (2000). The distributed human neural system for face perception. Trends in Cognitive Sciences, 4, 223-233.

Heinrich, S. P., Mell, D., \& Bach, M. (2009). Frequency-domain analysis of fast oddball responses to visual stimuli: a feasibility study. Int J Psychophysiol., 73, 287-289.

Heisz, J. J., Watter, S., \& Shedden, J. A. (2006). Automatic face identity encoding at the N170. Vision Research, 46, 4604-4614.

Helmholtz, H. (1867). "Handbuch der physiologischen Optik", in Allgemeine Encyklopddie der Physik volume 9, Ed. G Karsten (Leipzig: Voss)

Henson, R.N. (2016). Repetition suppression to faces in the fusiform face area: A personal and dynamic journey. Cortex, 80, 174-184.

Hermann, P., Grotheer, M., Kovacs, G., \& Vidnyanszky, Z. (2017). The relationship between repetition suppression and face perception. Brain Imaging and Behavior, 11(4), 1018-1028. 
Herrmann, C. S. (2001). Human EEG responses to 1-100 Hz flicker: resonance phenomena in visual cortex and their potential correlation to cognitive phenomena. Exp Brain Res, 137(34), 346-353.

Herzmann, G, Kunina, O., Sommer, W., \& Wilhelm, O. (2010). Individual differences in face cognition: brain-behavior relationships. J Cogn Neurosci. 22, 571-589.

Hildebrandt, A., Wilhelm, O., Schmiedek, F., Herzmann, G., \& Sommer, W. (2011). On the specificity of face cognition compared with general cognitive functioning across adult age. Psychol. Aging, 26, 701-715.

Hills, P. J., \& Lewis, M. B. (2018). The development of face expertise: Evidence for a qualitative change in processing. Cognitive Development, 48, 1-18.

Hobson, R. P., Ouston, J., \& Lee, A. (1988). What's in a face? The case of autism. British Journal of Psychology, 79(4), 441-453.

Hochberg, J., \& Galper, R. E. (1967). Recognition of faces: I. An exploratory study. Psychonomic Science, 9, 619-620.

Hole, G. J. (1994). Configurational factors in the perception of unfamiliar faces. Perception, 23, $65-74$.

Hönegger, C., Atteneder, C., Griesmayr, B., Holz, E., Weber, E., \& Sauseng, P. (2011). Neural correlates of visuo-spatial working memory encoding-An EEG study. Neuroscience Letters, $500,118-122$.

Hsiao, J.H., \& Cottrell, G. (2008). Two fixations suffice in face recognition. Psychological Science, 19, 998-1006.

Hughes, B. L., Camp, N. P., Gomez, J., Natu, V. S., Grill-Spector, K., \& Eberhardt, J. L. (2019). Neural adaptation to faces reveals racial outgroup homogeneity effects in early perception. Proceedings of the National Academy of Sciences, 116, 14532-14537.

Itier, R. J., \& Taylor, M. J. (2004). Effects of repetition and configural changes on the development of face recognition processes. Developmental Science, 7, 469-487.

Itz, M.L., Golle, J., Luttmann, S., Schweinberger, S.R, \& Kaufmann, J.M. (2017). Dominance of texture over shape in facial identity processing is modulated by individual abilities. $\mathrm{Br} J$ Psychol., 108, 369-396.

Jacques, C., \& Rossion, B. (2009). The initial representation of individual faces in the right occipito-temporal cortex is holistic: electrophysiological evidence from the composite face illusion. Journal of Vision. 11;9(6):8.1-16.

Jacques, C., Arripe, O., \& Rossion, B. (2007). The time course of the inversion effect during individual face discrimination. Journal of Vision, 7(8), (8), 3, 1-9. 
Jemel, B., Mottron, L., \& Dawson, M. (2006). Impaired Face Processing in Autism: Fact or Artifact? Journal of Autism and Developmental Disorders, 36, 91-106.

Jenkins, R., Dowsett, A. J., \& Burton, A. M. (2018). How many faces do people know? Proceedings. Biological Sciences, 285(1888).

Jenkins, R., White, D., Van Montfort, X., \& Burton, A. M. (2011). Variability in photos of the same face. Cognition, 121(3), 313-323

Jonas, J., Descoins, M., Koessler, L., Colnat-Coulbois, S., Sauvee, M., Guye, M., Vignal, J-P., Vespignani, H., Rossion, B., Maillard, L. (2012). Focal electrical intracerebral stimulation of a face-sensitive area causes transient prosopagnosia. Neuroscience, 222, 281-288.

Jonas, J., Rossion, B., Brissart, H., Frismand, S., Jacques, C., Colnat-Coulbois, S., Vespignani, H., Vignal, J.-P., Maillard, L. (2015). Beyond the core face-processing network: intracerebral stimulation of a face-selective area in the right anterior fusiform gyrus elicits transient prosopagnosia. Cortex, 72, 140-155.

Jonas, Jacques, Brissart, H., Hossu, G., Colnat-Coulbois, S., Vignal, J.-P., Rossion, B., \& Maillard, L. (2018). A face identity hallucination (palinopsia) generated by intracerebral stimulation of the face-selective right lateral fusiform cortex. Cortex, 99, 296-310.

Jonas, Jacques, Rossion, B., Krieg, J., Koessler, L., Colnat-Coulbois, S., Vespignani, H., ... Maillard, L. (2014). Intracerebral electrical stimulation of a face-selective area in the right inferior occipital cortex impairs individual face discrimination. NeuroImage, 99, 487-497.

Kaltwasser L, Hildebrandt A, Recio G, Wilhelm O, \& Sommer W. (2014). Neurocognitive mechanisms of individual differences in face cognition: a replication and extension. Cogn Affect Behav Neurosci., 14, 61-78.

Kang, E., Keifer, C. M., Levy, E. J., Foss-Feig, J. H., McPartland, J. C., \& Lerner, M. D. (2018). Atypicality of the N170 Event-Related Potential in Autism Spectrum Disorder: A Metaanalysis. Biological Psychiatry. Cognitive Neuroscience and Neuroimaging, 3, 657-666.

Kanwisher, N. (2017). The Quest for the FFA and Where It Led. The Journal of Neuroscience, 37, $1056-1061$.

Keitel, C., Quigley, C., \& Ruhnau, P. (2014). Stimulus-driven brain oscillations in the alpha range: entrainment of intrinsic rhythms or frequency-following response? J. Neurosci., 34, 1013710140.

Kelly, D.J., Liu, S., Lee, K., Quinn, P.C., Pascalis, O., Slater, A.M., \& Ge, L. (2009). Development of the other-race effect during infancy: evidence toward universality? J Exp Child Psychol. 104(1):105-14.

Kennerknecht, I., Grueter, T., Welling, B., Wentzek, S., Horst, J., Edwards, S, \& Grueter M. 
(2006). First report of prevalence of non-syndromic hereditary prosopagnosia (HPA). Am J Med Genet A. 140, 1617-1622.

Key, A.P. \& Corbett, B.A. (2020). The Unfulfilled Promise of the N170 as a Social Biomarker.Biol Psychiatry Cogn Neurosci Neuroimaging, 5, 342-353.

Keysers, C., Xiao, D.- K., Földiák, P., \& Perrett, D. I. (2001). The speed of sight. Journal of Cognitive Neuroscience, 13, 90-101.

Keysers, C. \& Perrett, D. I. (2002). Visual masking and RSVP reveal neural competition. Trends in Cognitive Science, 6, 120-125.

Kimura, M.,Widmann, A., \& Schröger, E. (2010). Human visual system automatically represents large-scale sequential regularities. Brain Res. 1317, 165-179.

Kimura, M., Schröger, E., \& Czigler I. (2011). Visual mismatch negativity and its importance in visual cognitive sciences. Neuroreport, 22, 669-673

Kirschstein T, Köhling R. (2009). What is the source of the EEG? Clin EEG Neurosci., 40, 146149.

Kok, R., Taubert, J., Van der Burg, E., Rhodes, G., \& Alais, D. (2017). Face familiarity promotes stable identity recognition: exploring face perception using serial dependence. $R$ Soc Open Sci. 4(3):160685.

Kovács G, Zimmer M, Bankó E, Harza I, Antal A, Vidnyánszky Z (2006) Electrophysiological correlates of visual adaptation to faces and body parts in humans. Cerebral Cortex, 16, 742753.

Kriegeskorte, N., Formisano, E., Sorger, B., \& Goebel R. (2007). Individual faces elicit distinct response patterns in human anterior temporal cortex. Proc Natl Acad Sci U S A, 104, 2060020605.

Kuefner, D., de Heering, A., Jacques, C., Palmero-Soler, E., \& Rossion, B. (2010). Early visually evoked electrophysiological responses over the human brain (P1, N170) show stable patterns of face-sensitivity from 4 years to adulthood. Frontiers in Human Neuroscience, 3(January), 67.

Laguesse, R., Dormal, G., Biervoye, A., Kuefner, D. \& Rossion, B. (2012). Extensive visual training in adulthood significantly reduces the face inversion effect. Journal of Vision, 12(14), $1-13$.

Langdell, T. (1978). Recognition of faces: an approach to the study of autism. Journal of Child Psychology and Psychiatry, and Allied Disciplines, 19(3), 255-268.

Laughery, K. R., Alexander, J. F., \& Lane, A. B. (1971). Recognition of human faces: Effects of target exposure, target position, pose position and type of photograph. Journal of Applied 
Psychology, 55, 477-483.

Lavallée, M.M., Gandini, D., Rouleau, I., Vallet, G.T., Joannette, M., Kergoat, M.-J., Busigny, T., Rossion, B., \& Joubert, S. (2016). A qualitative impairment in face perception in Alzheimer's disease: Evidence from a reduced face inversion effect. Journal of Alzheimer's Disease, 51, 1225-1236.

Leider, F., Daunizeau, J., Garrido, M.I., Friston, K.J., \& Stephan, K.E. (2013). Modelling trial-bytrial changes in the mismatch negativity. PLoS Computational Biology, 9:e1002911.

Leleu, A., Rekow, D., Poncet, F., Schaal, B., Durand, K., Rossion, B., \& Baudouin, J.-Y. (2019). Maternal odor shapes rapid face categorization in the infant brain. Developmental Science. e12877.

Leopold, D.A., O'Toole, A.J., Vetter, T., \& Blanz, V. (2001). Prototype-referenced shape encoding revealed by high-level aftereffects. Nat Neurosci. 4, 89-94.

Li, L., Miller, E.K., \& Desimone, R. (1993) The representation of stimulus familiarity in anterior inferior temporal cortex. J. Neurophysiol., 69, 1918-1929.

Liesefeld, H.R., \& Janczyk, M. (2019). Combining speed and accuracy to control for speedaccuracy trade-offs(?). Behav Res Methods. 51, 40-60.

Liu-Shuang, J., Norcia, A. M., \& Rossion, B. (2014). An objective index of individual face discrimination in the right occipito-temporal cortex by means of fast periodic oddball stimulation. Neuropsychologia, 52, 57-72.

Liu-Shuang, J., Ales, J., Rossion, B., Norcia, A.M. (2015). Separable effects of inversion and contrast reversal on face detection thresholds and response functions: a sweep VEP study. Journal of Vision. Feb 10;15(2).

Liu-Shuang, J., Torfs, K., \& Rossion, B. (2016). An objective electrophysiological marker of face individualisation impairment in acquired prosopagnosia with fast periodic visual stimulation. Neuropsychologia, 83, 100-113.

Lochy, A., Zimmermann, F., Laguesse, R., Willenbockel, V., Rossion, B. \& Vuong, Q. (2017). Does extensive training at individuating novel objects in adulthood lead to visual expertise? The role of facelikeness. Journal of Cognitive Neuroscience, 30, 449-467.

Lochy, A., Schiltz, C., \& Rossion, B. (2020). The right hemispheric dominance for face perception in preschool children depends on visual discrimination level. Developmental Science. Oct 16:e12914.

Logan, A.J., Wilkinson, F., Wilson, H.R., Gordon, G.E., \& Loffler, G. (2016). The Caledonian face test: A new test of face discrimination. Vision Research, 119, 29-41.

Logothetis, N.K. (2008). What we can do and what we cannot do with fMRI. Nature, 453, 869-878 
Luck, S. J. (2014). An introduction to the event-related potential technique. Cambridge, MA: MIT Press.

Makeig, S., Westerfield, M., Jung, T.P., Enghoff, S., Townsend, J., Courchesne, E., Sejnowski, T.J., 2002. Dynamic brain sources of visual evoked responses. Science, 295, 690-694.

Mardo, E., Avidan, G., \& Hadad, B.S. (2018). Adults' Markers of Face Processing Are Present at Age 6 and Are Interconnected Along Development. Perception, 47, 1002-1028.

Martin-Malivel, J., Mangini, M. C., Fagot, J., \& Biederman, I. (2006). Do humans and baboons use the same information when categorizing human and baboon faces? Psychological Science, 17(7), 599-607.

Mazard, A., Schiltz, C., \& Rossion, B. (2006). Recovery from adaptation to facial identity is larger for upright than inverted faces in the human occipito-temporal cortex. Neuropsychologia, 44(6), 912-922.

McCaffery, J.M., Robertson, D.J., Young, A.W., \& Burton, A.W. (2018). Individual differences in face identity processing. Cognitive Research: Principles and Implications, 3:21

McConachie, H. R. (1976). Developmental prosopagnosia. A single case report. Cortex, 12, 7682.

McKone, E., Crookes, K., Jeffery, L., \& Dilks, D. D. (2012). A critical review of the development of face recognition: experience is less important than previously believed. Cognitive Neuropsychology, 29(1-2), 174-212.

Meadows, J. C. (1974). The anatomical basis of prosopagnosia. Journal of Neurology, Neurosurgery \& Psychiatry, 37(5), 489-501.

Megreya, A.M., \& Bindemann, M. (2015). Developmental improvement and age-related decline in unfamiliar face matching. Perception. 44, 5-22.

Megreya, A.M., \& Burton, M. (2006) Unfamiliar faces are not faces: Evidence from a matching task. Memory \& Cognition, 34, 865-876.

Meyer, K, Schmitz, F, Wilhelm, O, \& Hildebrandt, A. (2019). Perceiving faces: Too much, too fast?-face specificity in response caution. J Exp Psychol Hum Percept Perform. 45, 16-38.

Milner, B. (1968). Visual recognition and recall after right temporal-lobe excision in man. Neuropsychologia, 6, 191-209.

Mondloch, C.J., Le Grand, R., \& Maurer, D. (2002). Configural face processing develops more slowly than featural face processing. Perception, 31, 553-66

Mondloch, C.J., Maurer, D., \& Ahola S. (2006). Becoming a face expert. Psychol Sci. 17, 930-934.

Munsters, N.M., van Ravenswaaij, H., van den Boomen, C., \& Kemner, C. (2019). Test-retest reliability of infant event related potentials evoked by faces. Neuropsychologia, 126, 20-26. 
Mur, M., Ruff, D. A., Bodurka, J., Bandettini, P. A., \& Kriegeskorte, N. (2010). Face-identity change activation outside the face system: "release from adaptation" may not always indicate neuronal selectivity. Cerebral Cortex, 20, 2027-2042.

Murphy, J., Gray, K.L., Cook, R. (2017). The composite face illusion. Psychon Bull Rev. 24, $245-$ 261.

Nemrodov, D., Jacques, C., Rossion, B. (2015). Temporal dynamics of repetition suppression to individual faces presented at a fast periodic rate. International Journal of Psychophysiology, $98,35-43$.

Nemrodov, D., Niemeier, M., Patel, A. \& Nestor, A. (2016). The neural dynamics of facial identity processing: insights from EEG-based pattern analysis and image reconstruction. dNeuro, 0358-17.2018.

Nestor, A., Plaut, D.C., \& Behrmann, M. (2011). Unraveling the distributed neural code of facial identity through spatiotemporal pattern analysis. Proc Natl Acad Sci U S A., 108, 9998-10003.

Nestor, A., Plaut, D. C., \& Behrmann, M. (2016). Feature-based face representations and image reconstruction from behavioral and neural data. Proceedings of the National Academy of Sciences of the United States of America, 113, 416-421.

Newport, C., Wallis, G., Reshitnyk, Y., \& Siebeck, U. E. (2016). Discrimination of human faces by archerfish (Toxotes chatareus). Scientific Reports, 6, 27523.

Nomi, J. S., \& Uddin, L. Q. (2015). Face processing in autism spectrum disorders: From brain regions to brain networks. Neuropsychologia, 71, 201-216.

Norcia, A. M., Appelbaum, L. G. G., Ales, J. M. J. M., Cottereau, B. R., \& Rossion, B. (2015). The steady-state visual evoked potential in vision research: a review. Journal of Vision, 15(6), 4.

Notbohm, A., \& Herrmann, C.S (2016). Flicker Regularity Is Crucial for Entrainment of Alpha Oscillations. Front Hum Neurosci.;10:503. eCollection 2016.

Nunez, P. L. \& Srinivasan, R. (2006). Electric Fields of the Brain. Oxford: Oxford University Press.

O'Toole, A. J., Price, T., Vetter, T., Bartlett, J. C., \& Blanz, V. (1999). 3D shape and 2D surface textures of human faces: the role of. Image and Vision Computing, 18, 9- 19.

O'Shea, R. P. (2015). Refractoriness about adaptation. Frontiers in Human Neuroscience, 9(38), $1-3$.

Orban de Xivry, J.-J., Ramon, M., Lefèvre, P., Rossion, B. (2008). Reduced fixation on the upper area of personally familiar faces following acquired prosopagnosia. Journal of Neuropsychology, 2, 245-268.

Palermo, P., Rossion, B., Rhodes, G., Laguesse, R., Tez, T., Hall, B., Albonico, A., Malaspina, M., 
Daini, R., Irons, J., Al-Janabi, S., Taylor, L.C., Rivolta, D., McKone, E. (2017). Do people have insight into their face recognition abilities? Quarterly Journal of Experimental Psychology, 70, 208-223.

Palermo, R., \& Rhodes, G. (2007). Are you always on my mind? A review of how face perception and attention interact. Neuropsychologia, 45, 75-92.

Parketny, J., Towler, J., \& Eimer M. (2015). The activation of visual face memory and explicit face recognition are delayed in developmental prosopagnosia. Neuropsychologia, 75, 538-547.

Parr, L.A., Heintz, M., \& Pradhan, G. (2008). Rhesus monkeys (Macaca mulatta) lack expertise in face processing. Journal of Comparative Psychology, 122, 390-402.

Pascalis, O., \& de Schonen, S. (1994). Recognition memory in 3- to 4-day-old human neonates. Neuroreport, 5, 1721-1724.

Pascalis, O., De Haan, M., \& Nelson, C. A. (2002). Is face processing species-specific during the first year of life ? Science, 296, 1321-1323.

Peirce, J. W., Leigh, A. E., daCosta, A. P. C., \& Kendrick, K. M. (2001). Human face recognition in sheep: lack of configurational coding and right hemisphere advantage. Behavioural Processes, 55(1), 13-26.

Perrett, D. I., Mistlin, A. J., Chitty, A. J., Smith, P. A. J., Potter, D. D., Broennimann, R., \& Harries, M. (1988). Specialized face processing and hemispheric asymmetry in man and monkey: evidence from single unit and reaction time studies. Behavioral Brain Research, 29, 245-258.

Peterson, M.F., \& Eckstein, M.P. (2012). Looking just below the eyes is optimal across face recognition tasks. Proc Natl Acad Sci U S A. 109(48):E3314-23.

Peterson, M.F., \& Eckstein, M.P. (2013). Individual differences in eye movements during face identification reflect observer-specific optimal points of fixation. Psychol Sci. 1;24(7):121625

Peykarjou, S., Hoehl, S., Pauen, S., \& Rossion, B. (2017). Rapid Categorization of Human and Ape Faces in 9-Month-Old Infants Revealed by Fast Periodic Visual Stimulation. Scientific Reports. 7(1):12526

Peykarjou, S., Pauen, S., \& Hoehl, S. (2014). How do 9-month-old infants categorize human and ape faces? A rapid repetition ERP study. Psychophysiology, 51(9):866-78.

Peykarjou, S., Pauen, S., \& Hoehl, S. (2015). 9-month-old infants recognize individual unfamiliar faces in a rapid repetition ERP paradigm. Infancy, 21, 288-311.

Picton, T.W. (1992). The P300 wave of the human event-related potential. J Clin Neurophysiol. 9 , 456-479.

Pitcher, D., Walsh, V., Yovel, G., \& Duchaine, B. (2007). TMS evidence for the involvement of 
the right occipital face area in early face processing. Current Biology, 17(18), 1568-1573.

Powell, G., Jones, C.R.G., Hedge, C., Charman, T., Happé, F., Simonoff, E., \& Sumner, P. (2019). Face processing in autism spectrum disorder re-evaluated through diffusion models. Neuropsychology, 33(4):445-461.

Puce, A., Allison, T, \& McCarthy, G. (1999). Electrophysiological studies of human face perception. III. Effects of top-down processing on face-specific potentials. Cerebral Cortex, $9,445-458$.

Purves, D., Morgenstern, Y., Wojtach, W.T. (2015). Perception and Reality: Why a Wholly Empirical Paradigm is Needed to Understand Vision. Front Syst Neurosci. 18;9:156

Quek, G., \& Rossion, B. (2017). Category-selective human brain processes elicited in fast periodic visual stimulation streams are immune to temporal predictability. Neuropsychologia, 104,182-200.

Quek, G., Nemrodov, D., Rossion, B., Liu-Shuang, J. (2018). Selective attention to faces in a rapid visual stream: Hemispheric differences in enhancement and suppression of category-selective neural activity. Journal of Cognitive Neuroscience, 30, 393-410.

Quek, G., Liu-Shuang, J., Goffaux, V., Rossion, B. (2018). Ultra-coarse, single-glance human face detection in a dynamic visual stream. NeuroImage, 16, 465-476.

Ramon, M., \& Gobbini, M. I. (2018). Familiarity matters: A review on prioritized processing of personally familiar faces. Visual Cognition, 26(3), 179-195.

Ramon, M., Dricot, L., Rossion, B. (2010). Personally familiar faces are perceived categorically in face-selective regions other than the FFA. European Journal of Neuroscience, 32, 1587-1598.

Rao, R.P., \& Ballard, D.H. (1999) Predictive coding in the visual cortex: a functional interpretation of some extra-classical receptive-field effects. Nat Neurosci. 2, 79-87.

Reed, C. L., Beall, P. M., Stone, V. E., Kopelioff, L., Pulham, D. J., \& Hepburn, S. L. (2007). Brief Report: Perception of Body Posture-What Individuals With Autism Spectrum Disorder might be Missing. Journal of Autism and Developmental Disorders, 37(8), 1576-1584.

Regan, D. (1966). Some characteristics of average steady-state and transient responses evoked by modulated light. Electroencephalography and Clinical Neurophysiology, 20, 238-248.

Regan, D. (1989). Human brain electrophysiology: Evoked potentials and evoked magnetic fields in science and medicine. New York: Elsevier.

Regan, D. \& Heron, J.R. (1969) Clinical investigation of lesions of the visual pathway: a new objective technique. J. Neurol. Neurosurg. Psychiatry, 32, 479-483.

Retter, T. \& Rossion, B. (2017). Visual adaptation reveals an objective electrophysiological measure of high-level individual face discrimination. Scientific Reports, 7(1):3269. 
Retter, T., Jiang, F., Webster, M., Rossion, B. (2018). Dissociable effects of inter-stimulus interval and presentation duration on rapid face categorization. Vision Research, 145, 1120.

Retter, T., Rossion, B. (2016b). Visual adaptation provides objective electrophysiological evidence of facial identity discrimination. Cortex, 80, 35-50.

Retter, T.L., Rossion, B. (2016). Uncovering the neural magnitude and spatio-temporal dynamics of natural image categorization in a fast visual stream. Neuropsychologia, 91, 928.

Rey, H.G., Gori, B., Chaure, F.J., Collavini, S., Blenkmann, A.O., Seoane, P., Seoane, E., Kochen S., Quian Quiroga, R. (2020). Single Neuron Coding of Identity in the Human Hippocampal Formation. Curr Biol. 30, 1152-1159.

Righi, G., Westerlund, A., Congdon, EL.., Troller-Renfree, S., \& Nelson, C.A. (2014). Infants' experience-dependent processing of male and female faces: insights from eye tracking and event-related potentials. Dev Cogn Neurosci., 8, 144-152.

Ritter, W., \& Vaughan, H.G. (1969). Averaged evoked responses in vigilance and discrimination: a reassessment. Science, 164, 326-328.

Ritter, W.H., Vaughan, H.G., \& Costa, L. (1968). Orienting and habituation to auditory stimuli: a study of short term changes in averaged evoked responses. Electroencephalogr Clin Neurophysiol., 25, 550-560.

Rolls, E.T., Baylis, G.C., Hasselmo, M.E., \& Nalwa, V. The effect of learning on the face selective responses of neurons in the cortex in the superior temporal sulcus of the monkey. Exp Brain Res. 76, 153-164.

Rose, F. E., Lincoln, A. J., Lai, Z., Ene, M., Searcy, Y. M., \& Bellugi, U. (2007). Orientation and affective expression effects on face recognition in Williams syndrome and autism. Journal of Autism and Developmental Disorders, 37, 513-522.

Rosenthal, G., Sporns, O., \& Avidan, G. (2017). Stimulus Dependent Dynamic Reorganization of the Human Face Processing Network. Cereb Cortex, 27, 4823-4834.

Rosset, D. B., Rondan, C., Fonseca, D. D., Santos, A., Assouline, B., \& Deruelle, C. (2008). Typical Emotion Processing for Cartoon but not for Real Faces in Children with Autistic Spectrum Disorders. Journal of Autism and Developmental Disorders, 38, 919-925.

Rossion, B. (2008). Picture-plane inversion leads to qualitative changes of face perception. Acta Psychologica, 128, 274-289.

Rossion, B. (2009). Distinguishing the cause and consequence of face inversion: the perceptual field hypothesis. Acta Psychologica, 132, 300-312.

Rossion, B. (2013). The composite face illusion: a window to our understanding of holistic face perception. Visual Cognition, 121, 139-253. 
Rossion, B. (2014). Understanding face perception by means of prosopagnosia and neuroimaging. Frontiers in Bioscience (Elite Ed.); 6-308-317.

Rossion, B. (2018a). Humans are visual experts at unfamiliar face recognition. Trends in Cognitive Sciences, 22, 471-472.

Rossion, B. (2018b). Damasio's error - Prosopagnosia with intact within-category object recognition. Journal of Neuropsychology, 12(3), 357-388.

Rossion, B. (2018c). Prosopdysgnosia? What could it tell us about the neural organization of face and object recognition? Cognitive Neuropsychology, 35, 98-101.

Rossion, B. (2020). Biomarkers of face perception in autism spectrum disorder: time to shift to rapid periodic visual stimulation with electroencephalography? Biological Psychiatry: Cognitive Neuroscience and Neuroimaging, 5, 258-260.

Rossion, B. \& Boremanse, A. (2011). Robust sensitivity to facial identity in the right human occipito-temporal cortex as revealed by steady-state visual-evoked potentials. Journal of Vision. 11(2):16, 1-21.

Rossion, B. \& Jacques, C. (2008). Does physical interstimulus variance account for early electrophysiological face sensitive responses in the human brain? Ten lessons on the N170. NeuroImage, 39, 1959-1979.

Rossion, B. \& Jacques, C. (2011). The N170 : understanding the time-course of face perception in the human brain. In The Oxford Handbook of ERP Components, Edited by S. Luck and E. Kappenman. Oxford University Press, pp. 115-142.

Rossion, B., \& Retter, T. (2015). Holistic face perception: mind the gap! Visual Cognition, 23, 379398.

Rossion, B., \& Michel, C. (2018). Normative data for accuracy and response times at the computerized Benton Facial Recognition Test (BFRT-c). Behavior Research Methods, 50, 2442-2460.

Rossion, B. \& Taubert, J. (2019). What can we learn about human individual face recognition from experimental studies in monkeys? Vision Research, 157, 142-158.

Rossion, B \& Retter, T.L. (2020). Face Perception. In: D. Poeppel, M.S. Gazzaniga \& G. Mangun (Eds). The Cognitive Neurosciences, Sixth Edition. The MIT Press.

Rossion, B., Caldara, R., Seghier, M., Schuller, A.-M., Lazeyras, F., Mayer, E. (2003). A network of occipito-temporal face-sensitive areas besides the right middle fusiform gyrus is necessary for normal face processing. Brain, 126, 2381-2395.

Rossion, B., Jacques, C., Jonas, J. (2018). Mapping face categorization in the human ventral occipito-temporal cortex with direct neural intracranial recordings. Annals of the New York 
Academy of Science, 1426, 5-24.

Rossion, B., Prieto, E.A., Boremanse, A., Kuefner, D., Van Belle, G. (2012). A steady-state visual evoked potential approach to individual face perception: effect of inversion, contrast-reversal and temporal dynamics. NeuroImage, 63, 1585-1600.

Rostalski, S.-M., Amado, C., \& Kovács, G. (2019). Repetition Suppression for Noisy and Intact Faces in the Occipito-Temporal Cortex. Frontiers in Psychology, 10, 1348.

Rousselet, G.A., Husk, J.S., Bennett, P.J., \& Sekuler, A.B. (2007). Single-trial EEG dynamics of object and face visual processing. Neuroimage, 36, 843-862.

Rousselet, G.A., Husk, J.S., Bennett, P.J., \& Sekuler, A.B. (2008). Time course and robustness of ERP object and face differences. J Vis.8(12):3.1-18

Russell R., Duchaine B., Nakayama K. (2009). Super-recognisers: people with extraordinary face recognition ability. Psychon. Bull. Rev., 16 252-257.

Russell, R., Biederman, I., Nederhouser, M., \& Sinha, P. (2007). The utility of surface reflectance for the recognition of upright and inverted faces. Vision Research, 47, 157-165.

Russell, R., Sinha, P., Biederman, I., \& Nederhouser, M. (2006). Is pigmentation important for face recognition? Evidence from contrast negation. Perception, 356, 749-759.

Sai, F. Z. (2005). The role of the mother's voice in developing mother's face preference: Evidence for intermodal perception at birth. Infant and Child Development, 14(1), 29-50.

Sangrigoli S, de Schonen S. (2004). Effect of visual experience on face processing: a developmental study of inversion and non-native effects. Dev Sci. 7(1):74-87.

Sawamura, H., Orban, G.A., Vogels, R (2006) Selectivity of neuronal adaptation does not match response selectivity: a single-cell study of the FMRI adaptation paradigm. Neuron 49, 307318.

Scherf, K. S., Behrmann, M., Minshew, N., \& Luna, B. (2008). Atypical development of face and greeble recognition in autism. Journal of Child Psychology and Psychiatry, 49, 838-847.

Scherf, K.S., Behrmann, M., Humphreys, K., \& Luna, B. (2007). Visual category-selectivity for faces, places and objects emerges along different developmental trajectories. Developmental Science, 10, 15-30.

Schiltz, C., \& Rossion, B. (2006). Faces are represented holistically in the human occipito-temporal cortex. NeuroImage, 32, 1385-1394.

Schiltz, C., Sorger, B., Caldara, R., Ahmed, F., Mayer, E., Goebel, R., \& Rossion B. (2006). Impaired Face Discrimination in Acquired Prosopagnosia Is Associated with Abnormal Response to Individual Faces in the Right Middle Fusiform Gyrus. Cerebral Cortex, 16, 57486. 
Schwarzer, G. (2000). Development of face processing: The effect of face inversion. Child Development, 71, 391-401.

Schweinberger, S. R., \& Neumann, M. F. (2016). Repetition effects in human ERPs to faces. Cortex, 80, 141-153.

Schweinberger, S. R., Huddy, V., \& Burton, M. (2004). N250r: A face-selective brain response to stimulus repetitions. NeuroReport, 15, 1501-1505.

Schweinberger, S. R., Pfütze, E.-M., \& Sommer, W. (1995). Repetition priming and associative priming of face recognition: Evidence from event-related potentials. Journal of Experimental Psychology: Learning, Memory, and Cognition, 21(3), 722-736.

Schweinberger, S.R., Pickerin,g E.C., Jentzsch, I, Burton, A.M.,\& Kaufmann, J.M. (2002). Eventrelated brain potential evidence for a response of inferior temporal cortex to familiar face repetitions. Brain Res Cogn Brain Res. 14, 398-409.

Scott, L.S., Shannon R.W., \& Nelson C.A. (2006). Neural correlates of human and monkey face processing by 9-month-old infants. Infancy. 10, 171-186

Sergent J., Ohta, S., \& MacDonald, B (1992). Functional neuroanatomy of face and object processing. Brain, 115, 15-36.

Sergent, J. (1989). Structural processing of faces. In A. W. Young \& H. D. Ellis (Eds.), Handbook of research on face processing (pp. 57-91). Amsterdam : Elsevier

Sergent, J., \& Signoret, J. L. (1992). Varieties of functional deficits in prosopagnosia. Cerebral Cortex, 2, 375-388

Sheehan, M. J., \& Nachman, M. W. (2014). Morphological and population genomic evidence that human faces have evolved to signal individual identity. Nature Communications, 5, 4800.

Sheehan, M. J., \& Tibbetts, E. A. (2011). Specialized face learning is associated with individual recognition in paper wasps. Science, 334, 1272-1275.

Sherrington, C. S. (1947). The Integrative Action of the Nervous System. New Haven, CT: Yale University Press.

Simson, R., Vaughn, H.G., Ritter, W. (1977). The scalp topography of potentials in auditory and visual discrimination tasks. Electroencephalogr Clin Neurophysiol., 42, 528-535.

Sorensen, T.A. \& Overgaard, M.S. (2018). Prosopagnosia or Prosopdysgnosia : Facing up to a change of concepts. 2018 10th International Conference on Knowledge and Smart Technology (KST).

Spaak, E, de Lange, F.P., Jensen, O. (2014). Local entrainment of $\alpha$ oscillations by visual stimuli causes cyclic modulation of perception. Journal of Neuroscience, 34(10):3536-44

Srinivasan, R., Russell, D.P., Edelman, G.M. \& Tononi, G. (1999). Increas synchronization of 
neuromagnetic responses during conscious perception. ., 19, 5435-5448.

Stacchi, L., Huguenin-Elie, E., Caldara, R., \& Ramon M. (2020). Normative data for two challenging tests of face matching under ecological conditions. Cogn Res Princ Implic. $19 ; 5(1): 8$.

Stacchi, L., Liu-Shuang, J., Ramon, M., \& Caldara, R. (2019a). Reliability of individual differences in neural face identity discrimination. NeuroImage, 189, 468-475.

Stacchi, L., Ramon, M., Lao, J., \& Caldara, R. (2019). Neural Representations of Faces Are Tuned to Eye Movements. Journal of Neuroscience, 39(21):4113-4123.

Stefanics, G., Heinzle, J., Horváth, A. A., \& Stephan, K. E. (2018). Visual mismatch and predictive coding: A computational single-trial ERP study. Journal of Neuroscience, 38, 4020-4030.

Summerfield, C., Trittschuh, E.H., Monti, J.M., Mesulam, M.-M., Egner, T., 2008. Neural repetition suppression reflects fulfilled perceptual expectations. Nat. Neurosci., 11, 10041006

Sutton, S., Braren, M., Zubin, J., John, E.R. (1965). Evoked-potential correlates of stimulus uncertainty, Science. 150, 1187-1188.

Tanaka, J.W., Curran, T., Porterfield, A.L., \& Collins, D. (2006). Activation of preexisting and acquired face representations: the N250 event-related potential as an index of face familiarity. J Cogn Neurosci. 18, 1488-1497.

Tang, J., Falkmer, M., Horlin, C., Tan, T., Vaz, S., \& Falkmer, T. (2015). Face Recognition and Visual Search Strategies in Autism Spectrum Disorders: Amending and Extending a Recent Review by Weigelt et al. PlosOne, 10, e0134439.

Tantam, D., Monaghan, L., Nicholson, H., \& Stirling, J. (1989). Autistic Children's Ability to Interpret Faces: A Research Note. Journal of Child Psychology and Psychiatry, 30, 623-63

Tavares, P. P., Mouga, S. S., Oliveira, G. G., \& Castelo-Branco, M. (2016). Preserved face inversion effects in adults with autism spectrum disorder: an event-related potential study. Neuroreport, 27, 587-592.

Taylor, M.J., Batty, M., \& Itier R.J. (2004). The faces of development: a review of early face processing over childhood. J Cogn Neurosci. 16, 1426-1442.

Todorovic, A., de Lange, F.P., (2012). Repetition suppression and expectation sup- pression are dissociable in time in early auditory evoked fields. J. Neurosci. 32, 13389-13395

Towler, J., Gosling, A., Duchaine, B., \& Eimer, M. (2012). The face-sensitive N170 component in developmental prosopagnosia. Neuropsychologia. 50, 3588-3599.

Tranel, D., Vianna, E., Manzel, K., Damasio, H., \& Grabowski T. (2009). Neuroanatomical correlates of the Benton Facial Recognition Test and Judgment of Line Orientation Test. $J$ 
Clin Exp Neuropsychol., 31, 219-233.

Tsao, D.Y., Moeller, S., \& Freiwald, W.A. (2008). Comparing face patch systems in macaques and humans. Proc Natl Acad Sci U S A. 105, 19514-9.

Turano, M.T., Marzi, T., \& Viggiano, M.P. (2016). Individual differences in face processing captured by ERPs. Int J Psychophysiol. 101, 1-8.

Turati, C., Bulf, H., \& Simion, F. (2008). Newborns' face recognition over changes in viewpoint. Cognition, 106, 1300-1321.

Turati, C., Macchi Cassia, V., Simion, F., \& Leo, I. (2006). Newborns' face recognition: The role of inner and outer facial features. Child Development, 77, 297-311.

Turati, C., Sangrigoli, S., Ruel, J., \& de Schonen, S. (2004). Evidence of the face inversion effect in 4-month-old infants. Infancy, 6, 275-297.

Van Der Geest, J. N., Kemner, C., Verbaten, M. N., \& Engeland, H. V. (2002). Gaze behavior of children with pervasive developmental disorder toward human faces: a fixation time study. Journal of Child Psychology and Psychiatry, 43, 669-678.

VanRullen, R., \& Reddy, L. (2019). Reconstructing faces from fMRI patterns using deep generative neural networks. Communications Biology, 2, 193

VanRullen, R., \& Reddy, L. (2019). Reconstructing faces from fMRI patterns using deep generative neural networks. Communications Biology, 2, 193.

Verosky, S.C., Zoner, K.A., Marble, C.W., Sammon, M.M., \& Babarinsa, C.O. (2020). Familiarization increases face individuation measured with fast periodic visual stimulation. Biol Psychol. 153:107883.

Verosky, S.C., Todorov, A., \& Turk-Browne, N.B. (2013). Representations of individuals in ventral temporal cortex defined by faces and biographies. Neuropsychologia 51, 2100-2108

Vettori, S., Dzhelyova, M., Van der Donck, S., Jacques, C., Steyaert, J., Rossion, B., \& Boets B. (2019b). Reduced neural sensitivity to rapid individual face discrimination in autism spectrum disorder. Neuroimage Clinical, 21, 101613.

Vettori, S., Jacques, C. Boets, B., Rossion, B. (2019a). Can the N170 be used as an electrophysiological biomarker indexing face processing difficulties in ASD? Biological Psychiatry: Cognitive Neuroscience and Neuroimaging, 4, 321-323.

Vida, M.D., Nestor, A., Plaut, D.C., \& Behrmann, M. (2017). Spatiotemporal dynamics of similarity-based neural representations of facial identity. Proc Natl Acad Sci U S A. 114, 388393.

Vizioli L., Rousselet, G.A., \& Caldara, R. (2010). Neural repetition suppression to identity is abolished by other-race faces. Proc Natl Acad Sci U S A. 107, 20081-20086. 
Vuong, Q.C., Willenbockel, V., Zimmermann, F.G.S., Lochy, A., Laguesse, R., Dryden, A. \& Rossion, B. (2017). Facelikeness matters: A parametric multipart object set to understand the role of spatial configuration in visual recognition. Visual Cognition, 24, 406-421.

Walter, W. G., Dovey, V. J., \& Shipton, H. (1946). Analysis of the electrical response of the human cortex to photic stimulation. Nature, $158,540-541$.

Walsh, K.S., McGovern, D.P., Clark, A., \& O'Connell, R.G. (2020). Evaluating the neurophysiological evidence for predictive processing as a model of perception. Ann NY Acad Sci. 1464, 242-268.

Wandell, B. A. (2011). The neurobiological basis of seeing words. Annals of the New York Academy of Sciences, 1224, 63-80.

Want, S. C., Pascalis, P., Coleman, M., \& Blades, M. (2003). Recognizing people from inner or outer parts of their faces: Developmental data concerning “unfamiliar” faces. British Journal of Developmental Psychology, 21, 125-135.

Warrington, E.K. (1984). Recognition Memory Test. Windsor: NFER-Nelson.

Webb, S. J., Jones, E. J. H., Merkle, K., Murias, M., Greenson, J., Richards, T., ... \& Dawson, G. (2010). Response to familiar faces, newly familiar faces, and novel faces as assessed by ERPs is intact in adults with autism spectrum disorders. International Journal of Psychophysiology, 77, 106-117.

Weigelt, S., Koldewyn, K., \& Kanwisher, N. (2012). Face identity recognition in autism spectrum disorders: a review of behavioral studies. Neuroscience \& Biobehavioral Reviews, 36, 10601084.

White, D., Kemp R.I, Jenkins, R., Matheson, M., \& Burton, A.M. (2014). Passport officers' errors in face matching. PLOS ONE 9:e103510.

Wieser, M.J., McTeague, L.M., \& Keil A. (2012). Competition effects of threatening faces in social anxiety. Emotion. 12, 1050-1060.

Wilhelm, O., Herzmann, G., Kunina, O., Danthiir, V., Schacht, A., \& Sommer, W. (2010). Individual differences in perceiving and recognizing faces-One element of social cognition. $J$ Pers Soc Psychol., 99, 530-548.

Wilmer, J.B. (2017). Individual Differences in Face Recognition: A Decade of Discovery. Current Directions in Psychological Science, 26, 225-230.

Wilmer, J. B., Germine, L., Chabris, C. F., Chatterjee, G., Williams, M., Loken, E., Nakayama, K., \& Duchaine, B. (2010). Human face recognition ability is specific and highly heritable. Proceedings of the National Academy of Sciences of the United States of America, 107, 52385241. 
Wilmer, J. B., Germine, L., Chabris, C. F., Chatterjee, G., Gerbasi, M., \& Nakayama, K. (2012). Capturing specific abilities as a window into human individuality: The example of face recognition. Cognitive Neuropsychology, 29, 360-392.

Xu, B., Liu-Shuang, J., Rossion, B., \& Tanaka, J. (2017). Individual Differences in Face Identity Processing with Fast Periodic Visual Stimulation. Journal of Cognitive Neuroscience, 29, 1368-1377.

Yan, X., Liu-Shuang, J., \& Rossion, B. (2019). Effect of face-related task on rapid individual face discrimination. Neuropsychologia, 129, 236-245.

Yan, X., Liu-Shuang, J., Rossion, B. (2019). Effect of face-related task on rapid individual face discrimination. Neuropsychologia, 129, 236-245.

Yan, X., Young, A.W., \& Andrews, T.J. (2017). The automaticity of face perception is influenced by familiarity. Atten Percept Psychophys. 79, 2202-2211.

Yin, R. K. (1969). Looking at upside-down faces. Journal of Experimental Psychology-Human Perception and Performance, 81, 141-145.

Young, A. W., \& Burton, A. M. (2018). Are We Face Experts? Trends in Cognitive Sciences, 22(2), $100-110$

Young, A. W., Hellawell, D., \& Hay, D. C. (1987). Configurational information in face perception. Perception, 16, 747-759.

Yovel, G., \& Kanwisher, N. (2005). The neural basis of the behavioral face-inversion effect. Current Biology, 15(24), 2256-2262.

Zhou, H., Melloni, L., Poeppel, D. \& Ding, N. (2016) Interpretations of Frequency Domain Analyses of Neural Entrainment: Periodicity, Fundamental Frequency, and Harmonics. Front. Hum. Neurosci. 10:274.

Zhu, M., \& Rozell, C.J., (2013). Visual nonclassical receptive field effects emerge from sparse coding in a dynamical system. PLoS Comput Biol 9, e1003191.

Zoefel, B., Ten Oever, S., \& Sack, A.T. (2018). The Involvement of Endogenous Neural Oscillations in the Processing of Rhythmic Input: More Than a Regular Repetition of Evoked Neural Responses. Front Neurosci. 12:95. 